\title{
Pressure-Driven Flows of Quemada Fluids in a Channel Lined with a Poroelastic Layer: a Linear Stability Analysis
}

\author{
Mohammad Pourjafar, and Kayvan Sadeghy* \\ Center of Excellence in Design and Optimization of Energy Systems (CEDOES), \\ School of Mechanical Engineering, College of Engineering, University of Tehran, \\ Tehran, Iran, P.O. Box: 11155-4563
}

\begin{abstract}
Laminar flow of a thixotropic fluid obeying the Quemada model is numerically investigated in a channel lined with a poroelastic layer saturated with a Newtonian fluid. Having assumed that the solid matrix in the poroelastic layer obeys the linear elastic model, basic flow/deformation were obtained in the main channel and also in the poroelastic layer using the biphasic mixture theory. The basic state was then subjected to infinitesimally-small, normal-mode perturbations and their vulnerability to poroelastic instability was studied based on the linear, temporal stability analysis. The eigenvalue problem so obtained was then solved numerically using the spectral collocation method. The main objective of the work was to investigate the role played by the pororelastic layer's parameters (i.e., porosity, flexibility, permeability, density, and thickness) on the stability picture. The roles played by the rheological behavior of the core fluid and the fluid flowing through the poroelastic layer were also investigated. Numerical results were obtained at low-permeability limit, typical of physiological systems, demonstrating that the effect of the layer's properties can be stabilizing or destabilizing depending on the rheological properties of the two fluids involved in the problem. In general, thixotropy was found to have a stabilizing effect on the core flow. The same was found to be true as to the effect of a fluid's shear-thinning. The analysis also served to show that the effect of a fluid's yield stress might also be stabilizing.
\end{abstract}

Keywords: Poroelastic layer, Quemada model, linear stability, Poiseuille flow, biphasic mixture theory.

\section{Introduction}

Pressure-driven flow of viscous fluids through a channel bounded by porous layers is encountered in a variety of engineering systems. One can mention, for example, extraction of crude oils, pollution of surface and ground water, separation processes, fuel cells, heat pipes, and porous bearings, among others [1-7]. Further interest in this fascinating type of flow originates from its biomechanical applications. Blood vessels, for example, are covered with a poroelastic layer called glycocalyx serving different purposes $[8,9]$. The articular cartilage in our knee joint is another example in which this type of flow naturally occurs [9]. For reasons like these, the flow has received much attention in the past in the academic world. For understandable reasons, early works in this area were primarily concerned with the

* Corresponding author.

Tel.: +98 2161119927 ;

fax: +98 2188013029 .

E-mail address: sadeghy@ut.ac.ir (K. Sadeghy) 
Newtonian/Newtonian (N/N) case. In fact, the same fluid was assumed to be flowing through the main channel and the porous layer [1-7]. These works can be classified into three different categories based on the characteristics of the layer used in the analysis: i) the layer is permeable but inelastic, ii) the layer is impermeable but elastic, iii) the layer is both permeable and elastic (i.e., poroelastic).

As to the first type of layers (i.e., merely porous layers), one can mention the seminal work of Beavers et al [10]. They experimentally showed that in the channel shown schematically in Fig. 1, permeability has a destabilizing effect on the main flow. Their conclusion was later corroborated theoretically by Sparrow et al [11] who relied on the Darcy's law to describe the creeping flow passing through the porous layer. Chang et al [12] also relied on the Darcy model in their analysis and theoretically showed that for slightly-permeable layers, the flow in the main channel becomes unstable provided that it is sufficiently thick. For thin layers, however, they predicted that instability was controlled by the thickness of the fluid layer instead of the porous layer. Liu et al [13] demonstrated that the bi-modal behavior predicted by Chang et al [12] is true even for highly permeable layers provided that use is made of the Brinkman model instead of the Darcy model in the porous layer. Interestingly, the theoretical results reported by Socio et al [14] shows no bi- or tri-modal behavior in the neutral stability curve. The experimental data obtained by Silin et al [15] also showed no sign of bi-modal stability curve. Tilton and Cortelezzi [16] argued that the prediction of bi- and tri-modal neutral stability curves in Refs. 12 and 13 is an artifact of using inappropriate length scales when making the governing equations dimensionless. They also demonstrated that the case in which only one wall is porous is more stable than the case having two symmetric porous walls. In a more recent work, Deepu et al [17] demonstrated that anisotropy and inhomogeneity of the porous layer can be used as an efficient tool for controlling transition to turbulence in poroelastic channels.

As to the second type of layers (i.e., simply elastic layers), one can mention the interesting work carried out by Pierucci and Morales [18] in a channel lined with an impermeable but linearly-elastic layer. They predicted that at sufficiently high Reynolds numbers (typical of boundary layer flows) wall compliance has a stabilizing effect on the flow. Gkanis and Kumar [19] showed that the response becomes totally different if instead of the linear elastic model use is made of the nonlinear neo-Hookean model. It was shown that for this particular solid model a depth-dependent first normal-stress-difference arises in the base state (even under creeping-flow conditions) leading to a shortwave instability. In a more recent work, Pourjafar and Sadeghy [20] resorted to the Mooney-Rivlin model and reached to the conclusion that a negative second normal-stress-difference has a stabilizing effect on the flow. They also showed that, in order to avoid discontinuity in the neutral stability curve, inertial terms should always be included in the analysis no matter how small the Reynolds number might be. Further interest in this type of instability arises from the fact that it can dramatically increase the rate of mass transfer even for creeping flows [21].

The third type of layers (i.e., the poroelastic layers) has not been investigated to the same extent in the past regardless of the fact that they are quite common in physiological systems [22,23]. To this should be added the fact that, poroelastic layers of low permeability are increasingly being considered as dragreducing coatings to improve the aerodynamic performance of next-generation airplanes. Based on current knowledge [24], it is already known that, as far as Tollmien-Schlichting (TS) modes are concerned, poroelastic coatings have a stabilizing effect on the boundary layer formed above a wing. Interestingly, the stabilizing effect of poroelastic coatings on boundary layer flows was shown to be 
caused by their compliance which more than compensates the destabilizing effect of their permeability [24]. As to the confined flows, Albers [25] relied on a one-dimensional stability analysis for a Newtonian fluid flowing through a channel completely occupied with a poroelastic material. Although Albers' work [25] is not so relevant to the present study - she was mainly concerned with the effect of mass adsorption on the stability picture - it served to show that complications might arise (even in one-dimensional stability studies) when permeability and flexibility compete to stabilize or destabilize a given flow.

To our surprise, linear stability of channel flows for a Newtonian fluid flowing above a poroelastic layer saturated with the same or another Newtonian fluid has not previously been studied. In fact, as far as we are aware of, the only work dealing with the poroelastic instability of Newtonian fluids in such channels is the one carried out by Samanta et al [26]. But, in their short two-page conference paper, there is no poroelastic data presented whatsoever (even for the basic flow). There is also not much details in their short paper as to how the solid matrix has been modelled and/or how the flow in the poroelastic layer has been resolved. (It is speculated that due to their link with the European Commission PEL-skin project [27] perhaps proprietary issues were involved.) What is obvious is that there is a huge lack of knowledge in the open literature when it comes to the stability of Newtonian liquids flowing over a poroelastic layer in channel flows. With this in mind, in the present work, we intend to study the instability of Newtonian fluids in a two-dimensional channel lined with a poroelastic layer. For the work to be applicable to biomechanical systems, we intend to allow the fluid flowing through the core of the channel to be nonNewtonian. Instability of non-Newtonian fluids in permeable and compliant channels has been investigated in separate works in the past [28-30], but there is no such data available in the literature for poroelastic channels. Such data are important because physiological fluids such as blood, mucus, and synovia are known to exhibit a variety of non-Newtonian behavior, chief among them are their time- and shear-dependent viscosity. The same is true for many industrial fluids such as drilling muds, waxy crude oils, lubricants, and the like. Fortunately, the rheological model adopted for the analysis in the present work (i.e., the Quemada model) allows both effects to be addressed for certain range of model parameters. As to the rheology of the fluid flowing through the porous layer, in compliance with real physiological systems, the fluid is assumed to obey the Newtonian model.

For ease of analysis, we assume that the deformation in the solid skeleton of the poroelastic layer is infinitesimally small, so much so that its porosity, permeability, and elasticity all remain virtually constant during the deformation. Thus, a linear elastic model is used to represent the deformation of the solid matrix. For the work to be as comprehensive as possible, we have decided to include all inertia terms in the analysis. As to the poroelastic layer, we have decided to employ the biphasic mixture theory for describing its flow/deformation and also its interfacial boundary conditions. This theory is known to well describe poroelastic materials in physiological systems [31,32].

Our main objective in this work is to figure out the comparative role played by the properties of the poroelastic layer (say, its porosity, flexibility, permeability, density, and thickness) on the poroelastic stability of the main flow. Since in biomechanical systems [22,23] and drug-reduction applications [27] porolelastic layers are often realized to be quite dense, we assume that the permeability of the layer is small. It is shown that, unlike the boundary-layer flows where poroelasticity monotonically stabilizes the flow [24], in channel flows poroelastic coatings can have a stabilizing or destabilizing effect on the main flow depending on the physical properties of the layer and the rheology of the working fluids [33]. To reach its objectives, the work is organized as follows: In the next section, we formulate the mathematical framework for our fluid-solid-interaction (FSI) problem by developing the equations governing the basic 
flow/deformation in the poroelastic layer and in the main channel. Having obtained the basic flow/deformation, we then proceed with investigating their vulnerability to infinitesimally-small perturbations through invoking a linear, temporal, stability analysis. The numerical method of solution (i.e., the spectral method) is described next in some details. Numerical results are then presented accompanied by a discussion on their physical significance. The work is concluded by highlighting its major findings.

\section{Mathematical Formulations}

Figure 1 schematically shows the flow configuration used in the present study. As can be seen in this figure, we are studying the plane Poiseuille flow of a fluid between two rigid plates a distance H+R apart with the lower plate lined by a poroelastic gel having a thickness of $\mathrm{R}$. The length of the channel is denoted by $\mathrm{L}$ which is assumed to be much larger than $\mathrm{H}$ and $\mathrm{R}$ such that the core flow can safely be taken as fully-developed (i.e., there is no x-dependency in any of the terms except the pressure). The flow is occurring from left to right under the influence of a common, constant, negative pressure gradient, $\partial \mathrm{p} / \partial \mathrm{x}$. The flow generated this way is laminar and occurring under isothermal conditions with no influence from the gravity. For ease of analysis, and also because of the pioneering nature of the present work, the porosity/permeability of the layer is assumed to be isotropic and homogeneous.

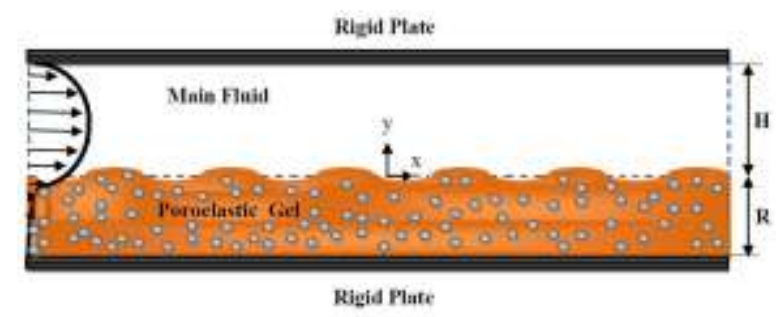

Figure 1: Schematic showing the two-dimensional flow geometry.

In this figure, the interface (which is initially flat) has been perturbed by a travelling wave.

Since we are dealing with a fluid-solid-interaction (FSI) problem, we need to develop separate governing equations for the main channel and for the poroelastic layer. These equations become coupled through invoking appropriate boundary conditions at the interface. We rely on the Cartesian coordinate system for developing the mathematical formulations for the main channel and for the porous layer. The origin of the coordinate system is placed at the interface (see Fig. 1).

\subsection{Governing Equations for the Poroelastic Region}

As to the equations governing the fluid phase and the solid phase in the poroelastic layer, we rely on the mixture theory. Based on this theory, a poroelastic material is treated like a continuum mixture of two inter-penetrating phases each having its own density and displacement. This is tantamount to saying that, each spatial point in the mixture is occupied simultaneously by both phases. In essence, this theory relies on the "principle of effective stress" for describing the mechanical behavior of a material [32]. Based on this principle, the total stress at any given point in the material is the sum of the solid stress and the fluid pressure. This principle is based on the premise that in a fluid-saturated poroelastic material any strain imposed on the material affects the fluid pressure in addition to affecting the solid's stress. That is to say that, any change in the fluid pressure gives rise to a change in the solid stress, and vice versa. This 
biphasic mixture theory has been found to well describe the behavior of poroelastic materials such as bone, cartilage, intervertebral disks, and certain joints in human body [32]. Based on this theory, the conservation of mass and momentum for each phase in the poroelastic material (treated as a mixture) can be written as [32]:

$$
\begin{aligned}
& \frac{\partial \varphi_{\mathrm{f}}}{\partial \mathrm{t}}+\nabla \cdot\left(\varphi_{\mathrm{f}} \mathbf{V}_{\mathrm{f}}\right)=0 \\
& \varphi_{\mathrm{f}} \rho_{\mathrm{f}}\left(\frac{\partial \mathrm{V}_{\mathrm{f}}}{\partial \mathrm{t}}+\left(\mathrm{V}_{\mathrm{f}} \cdot \nabla\right) \mathrm{V}_{\mathrm{f}}\right)=-\varphi_{\mathrm{f}} \nabla \mathrm{p}_{\mathrm{avg}}+\nabla \cdot \tau_{\mathrm{f}}+\varphi_{\mathrm{f}} \frac{\mu_{\mathrm{f}}}{\mathrm{K}}\left(\mathrm{V}_{\mathrm{s}}-\mathrm{V}_{\mathrm{f}}\right) \\
& \frac{\partial \varphi_{\mathrm{s}}}{\partial \mathrm{t}}+\nabla \cdot\left(\varphi_{\mathrm{s}} \mathrm{V}_{\mathrm{s}}\right)=0 \\
& \varphi_{\mathrm{s}} \rho_{\mathrm{s}}\left(\frac{\partial \mathrm{V}_{\mathrm{s}}}{\partial \mathrm{t}}+\left(\mathrm{V}_{\mathrm{s}} \cdot \nabla\right) \mathrm{V}_{\mathrm{s}}\right)=-\varphi_{\mathrm{s}} \nabla \mathrm{p}_{\mathrm{avg}}+\nabla \cdot \tau_{\mathrm{s}}-\varphi_{\mathrm{f}} \frac{\mu_{\mathrm{f}}}{\mathrm{K}}\left(\mathrm{V}_{\mathrm{s}}-\mathrm{V}_{\mathrm{f}}\right)
\end{aligned}
$$

where $\rho_{\mathrm{f}}$ is the density of the fluid in the poroelastic zone, $\varphi_{\mathrm{f}}$ is the volume fraction of the fluid in this layer, $\varphi_{\mathrm{s}}\left(=1-\varphi_{\mathrm{f}}\right)$ is the volume fraction of the solid in the poroelastic layer, $\mathrm{p}_{\mathrm{avg}}$ is the average pressure in this layer, $\mathrm{K}$ is the layer's permeability, $\mathbf{V}_{\mathrm{f}}$ is the fluid's velocity vector in the poroelastic layer, $\mathbf{V}_{\mathrm{s}}$ is the solid's velocity vector in the poroelastic layer, and $\tau_{\mathrm{f}}$ and $\tau_{\mathrm{s}}$ are the stress tensors for each phase in this layer. Since the fluid flowing through the poroelastic layer is Newtonian, we have:

$$
\tau_{\mathrm{f}}=\mu_{\mathrm{f}}\left(\nabla \mathbf{V}_{\mathrm{f}}+\nabla \mathbf{V}_{\mathrm{f}}^{\mathrm{T}}\right)
$$

where $\mu_{\mathrm{f}}$ is the viscosity of the fluid in this layer. As to the stress tensor needed to represent the solid phase in the poroelastic layer, since deformations and deformation gradients encountered in physiological systems and drag reduction applications are typically very small, like Wei et al [31] we resort to the linear elasticity theory for describing the deformation of the solid matrix; that is [32]:

$$
\tau_{\mathrm{s}}=\mu_{\mathrm{s}}\left(\nabla \mathbf{X}_{\mathrm{s}}+\nabla \mathbf{X}_{\mathrm{s}}^{\mathrm{T}}\right)+\chi\left(\nabla \cdot \mathbf{X}_{\mathrm{s}}\right) \mathbf{I}
$$

where $\mathbf{I}$ is the identity tensor. In this equation $\mathbf{X}_{\mathrm{s}}$ is the displacement vector which is related to the velocity field of the solid phase through the relationship: $\mathbf{V}_{\mathrm{s}}=\mathrm{d} \mathbf{X}_{\mathrm{s}} / \mathrm{dt}$. It is worth-mentioning that in this equation, $\mu_{\mathrm{s}}$ is the solid's shear modulus. On the other hand, $\chi$ is a Lame's constant related to the Poisson's ratio $(v)$ and the Young's modulus of Elasticity (E) through the relationship: $\chi=\mathrm{Ev} /[(1+v)(1-2 v)]$; see Ref. 32. Although, generally-speaking, $\chi$ is non-zero, in Ref. 23 it has been shown that the influence of this term is quite negligible in situations where the deformation is very small. Since in our linear stability analysis, the deformation is infinitesimally small in the transverse direction, one might be tempted to safely drop this term from the solid model right from the beginning. To make the work as comprehensive as possible, we have decided to keep this term in our mathematical developments. 


\subsection{Governing Equations for the Main Channel}

The fluid in the main channel is non-Newtonian, and so we have to start from the Cauchy equations of motion together with the continuity equation as the equations governing the flow; that is:

$$
\rho \frac{\mathrm{DU}}{\mathrm{Dt}}=-\nabla \mathrm{p}+\nabla \cdot \tau
$$

$\nabla \cdot \mathbf{U}=0$,

where $\mathrm{D} / \mathrm{Dt}$ is the material derivative, $\mathbf{U}$ is the velocity vector in the main channel, $\mathrm{p}$ is the isotropic pressure, and $\rho$ is the fluid's density. The stress tensor, $\tau$, must be related to the velocity field through an appropriate constitutive equation. The viscosity of physiological fluids such as synovia [34] and blood [35] are known to be simultaneously both shear- and time-dependent. This is also true for industrial fluids such as waxy crude oils [36]. These fluids are also known to be somewhat viscoelastic although it should be conceded that under their normal working conditions, their elasticity is of secondary effect, if any. Thus, generalized Newtonian fluid models (GNFs), valid for purely-viscous non-Newtonian fluids, are often realized to be more than adequate for describing their rheology. One can mention, for example, the Quemada model [37,38] which is known to accurately fit data for blood and many other physiological fluids [39]. Being a GNF material, the stress tensor for this robust fluid model reads as:

$$
\tau=\eta(\mathrm{t}, \dot{\gamma}) 2 \mathbf{D}
$$

where $2 \mathbf{D}$ is the rate-of-deformation tensor with $\dot{\gamma}=\sqrt{2 \mathrm{D}_{\mathrm{ij}} \mathrm{D}_{\mathrm{ij}}}$ being its norm. In this fluid model, the time-dependency is introduced through invoking a scalar function, $\mathrm{S}(\mathrm{t}, \dot{\gamma})$, called the structural parameter. The structural parameter lies in the range of 0 to 1 with zero denoting complete structure breakdown and one denoting complete structure rebuild. In the Quemada model, the structural parameter satisfies the following kinetic equation [37,38]:

$$
\frac{\mathrm{DS}}{\mathrm{Dt}}=\mathrm{a}(1-\mathrm{S}) \dot{\gamma}^{\mathrm{m}}-\mathrm{bS} \dot{\gamma}^{\mathrm{n}}
$$

where $\mathrm{D} / \mathrm{Dt}$ is the material derivative. In this equation, the coefficients "a" and "b" are model parameters controlling the rate of structure rebuild and structure destruction, respectively. (It is worth-mentioning that for fluids labelled "thixotropic", structure rebuild occurs at a pace much lower than that for the structure destruction [40].) The apparent viscosity of the Quemada fluid is then related to the structural parameter through the following relationship [37,38]:

$$
\eta(S, \dot{\gamma})=\mu_{\infty}(1+\varepsilon S)^{\beta} .
$$

The viscosity function is seen to comprise a permanent part denoted by $\mu_{\infty}$ (which corresponds to $S=0$ and is called the infinite-shear viscosity) and a time-dependent part controlled by $\varepsilon$ and the power-law exponent $\beta$. It is easy to see that by simply setting $\varepsilon=0$ the Quemada model reduces to the Newtonian model with a viscosity equal to $\mu_{\infty}$. Indeed, for the model to represent a time-dependent fluid it is required that: $\varepsilon \neq 0$ and $\beta \neq 0$. Furthermore, among "a" and "b", at least one of them should be non-zero otherwise $\mathrm{S}$ (or, equivalently, the viscosity) would remain a constant. 
A reduced form of the Quemada model is the Moore model [41] which corresponds to: $\mathrm{m}=0, \mathrm{n}=1$, and $\beta=1$; this model reads as:

$$
\begin{aligned}
& \frac{\mathrm{DS}}{\mathrm{Dt}}=\mathrm{a}(1-\mathrm{S})-\mathrm{bS} \dot{\gamma}, \\
& \eta(\mathrm{S}, \dot{\gamma})=\mu_{\infty}(1+\varepsilon S) .
\end{aligned}
$$

The viscosity of a Moore fluid comprises a constant part $\left(\mu_{\infty}\right)$ and a thixotropic part, $\left(\varepsilon \mu_{\infty} S\right)$, where $\varepsilon$ can be related to the zero-shear viscosity $\left(\mu_{\infty}\right)$ through the relationship: $\varepsilon=\left(\mu_{0}-\mu_{\infty}\right) / \mu_{\infty}$ with $\Delta \mu=\mu_{0}-\mu_{\infty}$ called the viscosity gap. Since for thixotropic fluids we have: $\mu_{0}>\mu_{\infty}$, one can conclude that in the Moore model the viscosity-gap ratio, $\varepsilon$, is a positive number. It is worth-mentioning that Moore model is widely used for fundamental studies trying to delineate the qualitative role played by thixotropy in any given flow-particularly when dealing with complex fluids such as ceramic slurries, debris, and metal paints [42-45]. To see how the material constants (a,b) appearing in the Moore model control its thixotropic behavior, we can integrate Eq. 10a in simple shear flow (i.e., where $\dot{\gamma}=$ Const. ) in order to obtain:

$\mathrm{S}(\mathrm{t})=\mathrm{S}_{\mathrm{e}}-\left(\mathrm{S}_{\mathrm{e}}-\mathrm{S}_{\mathrm{i}}\right) \exp [-(\mathrm{a}+\mathrm{b} \dot{\gamma}) \mathrm{t}]$

where $S_{i}$ is the structural parameter at $t=0$ and $S_{e}$ is the structural parameter when equilibrium is reached. If we assume that under equilibrium there is no structure left in the fluid (i.e., $S_{e}=0$ ) we obtain:

$\mathrm{S}(\mathrm{t})=\mathrm{S}_{\mathrm{i}} \exp [-(\mathrm{t} / \lambda)]$

where,

$\lambda=\frac{1}{\mathrm{a}+\mathrm{b} \dot{\gamma}}$,

is the decay time of the Moore fluid. If we make $\lambda$ dimensionless through multiplying it by "a" we end up with the dimensionless decay time defined as:

$$
\Lambda=\frac{1}{1+(\mathrm{b} / \mathrm{a}) \dot{\gamma}}
$$

where $\mathrm{k}=\mathrm{b} / \mathrm{a}$ is the characteristic time of the Moore fluid with its inverse sometimes called the characteristic shear rate [see Ref. 44] -it can easily be shown that at this particular shear rate, the viscosity of a Moore fluid is equal to $\left(\mu_{0}+\mu_{\infty}\right) / 2$. Moore fluids for which $\Lambda$ is large can be regarded as highly-thixotropic because they need a longer time to forget their initial viscosity before reaching their equilibrium viscosity. This notion can readily be seen in Fig. 2, which is a plot of Eq. 11a. As can be seen in this figure, when $\Lambda$ is large, a Moore fluid finds its equilibrium structural parameter quite slowly meaning that it is exhibiting strong thixotropic behavior. On the other hand, when $\Lambda$ is small, the fluid is practically behaving like a highly shear-thinning fluid as it finds its equilibrium structural configuration quite fast (see Fig. 2). 


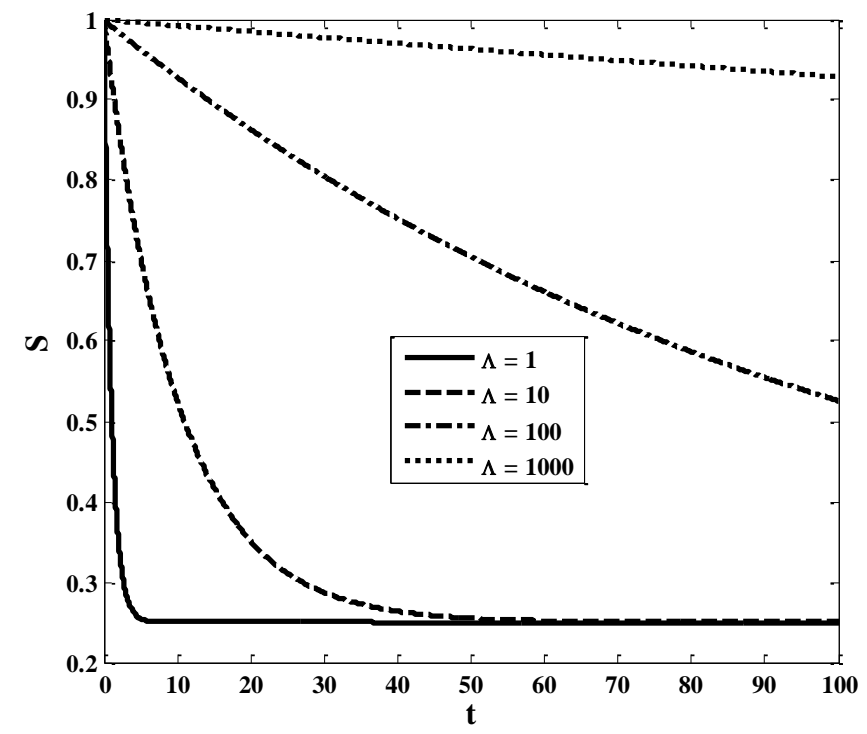

Figure 2: Effect of the b/a ratio (as represented by $\Lambda$ ) on the structure relaxation in simple shear for Moore fluids obtained for $S_{e}=0.25$.

Based on Eq. 11d, for $\Lambda$ to be large it suffices that the ratio $\mathrm{k}=\mathrm{b} / \mathrm{a}$ is sufficiently small. This means that, Moore fluids having small b/a ratios are more likely to exhibit strong thixotropic effects. Having said this, it should be conceded that, in practice, it is the ratio between the decay time of the fluid and the characteristic time of the flow which determines the severity of any thixotropic behavior. The characteristic time of the flow can be defined as: $\mathrm{T}=\mu_{\infty} / \mathrm{HG}$, where $\mathrm{G}$ is the pressure gradient. Therefore, thixotropic effects are expected to have a significant influence on any particular flow if, and only if, the ratio $\lambda / \mathrm{T}$ is sufficiently large. (In Ref. 44 , this ratio was dubbed the name of Deborah number but denoted by the abbreviation Db instead of De to convey the fact that a Moore fluid is an inelastic fluid.) For a given $\mathrm{T}$, for the $\lambda / \mathrm{T}$ ratio to be large it suffices that $\lambda$ is large, and for $\lambda$ to be large, it is required that the $k=b / a$ ratio is sufficiently small (say, for a given "a"). As such, the following dimensionless number, called the thixotropy number (Tx) can be used as a good measure to highlight the importance of thixotropy:

$\mathrm{Tx}=\frac{\mathrm{k}}{\mathrm{T}}=\frac{\mathrm{b} / \mathrm{a}}{\mu_{\infty} / \mathrm{HG}}$.

Results obtained at very small Tx numbers highlight thixotropic effects. On the other hand, results obtained at large Tx numbers highlight shear-thinning effects, as shown in Appendix A. (As a matter of fact, for certain range of parameters, the Moore model can also represent viscoplastic fluids; see Appendix B.)

\subsection{Boundary Conditions}

To close the problem, we need appropriate boundary conditions. Based on the biphasic mixture theory, at the interface between the poroelastic layer and the core fluid we should have [31,32]: 


$$
\begin{aligned}
& \mathbf{n} \cdot \mathbf{U}=\mathbf{n} \cdot\left(\Lambda_{\mathrm{s}} \varphi_{\mathrm{s}} \mathbf{V}_{\mathrm{s}}+\Lambda_{\mathrm{f}} \varphi_{\mathrm{f}} \mathbf{V}_{\mathrm{f}}\right) \\
& \mathbf{n} \cdot \sigma=\mathbf{n} \cdot\left(\sigma_{\mathrm{f}}+\sigma_{\mathrm{s}}\right) \\
& \sigma_{\mathrm{f}}=-\varphi_{\mathrm{f}} \mathrm{p}_{\mathrm{avg}} \mathbf{I}+\tau_{\mathrm{f}}=\varphi_{\mathrm{f}} \sigma \\
& \sigma_{\mathrm{s}}=-\varphi_{\mathrm{s}} \mathrm{p}_{\mathrm{avg}} \mathbf{I}+\tau_{\mathrm{s}}=\varphi_{\mathrm{s}} \sigma
\end{aligned}
$$

where $\boldsymbol{\sigma}=-\mathrm{p} \mathbf{I}+\boldsymbol{\tau}$ is the total stress tensor for the fluid in the core region, $\mathbf{n}$ is the unit vector normal to the interface, and $p_{a v}$ is the average pressure in the poroeastic material. In the above equations, $\Lambda_{\mathrm{f}}=\rho_{\mathrm{f}} / \rho$ and $\Lambda_{\mathrm{s}}=\rho_{\mathrm{s}} / \rho$ are the fluid density ratio and the solid density ratio, respectively. On the surface of the lower rigid plate (i.e., the plate on which the poroelastic layer is fixed, glued, or coated) we impose the no-slip and no-penetration conditions for the fluid phase, and the no-displacement condition for the solid phase; that is:

$@ \mathrm{y}=-\mathrm{R} ;\left\{\begin{array}{l}\mathrm{u}_{\mathrm{f}}=\mathrm{v}_{\mathrm{f}}=0 \\ \mathrm{x}_{\mathrm{x}}=\mathrm{X}_{\mathrm{y}}=0\end{array}\right.$

On the surface of the upper rigid plate we impose the no-slip and no-penetration conditions; that is:

@ $\mathrm{y}=\mathrm{H} ; \mathrm{u}=\mathrm{v}=0$

And, this completes our development of the governing equations.

\subsection{Dimensionless Equations}

In order to work with dimensionless parameters (shown by asterisks) we substitute:

$$
\begin{aligned}
& \mathrm{x}^{*}, \mathrm{y}^{*}=\frac{\mathrm{x}, \mathrm{y}}{\mathrm{H}} ; \mathrm{X}^{*}, \mathrm{Y}^{*}=\frac{\mathrm{X}, \mathrm{Y}}{\mathrm{H}} ; \mathrm{h}^{*}=\frac{\mathrm{R}}{\mathrm{H}} ; \mathrm{t}^{*}=\frac{\mathrm{t}}{\mu_{\infty} / \mathrm{GH}} ; \mathrm{u}^{*}, \mathrm{v}^{*}=\frac{\mathrm{u}, \mathrm{v}}{\mathrm{GH}^{2} / \mu_{\infty}} ; \mathrm{p}^{*}=\frac{\mathrm{p}}{\mathrm{HG}} ; \tau_{\mathrm{ij}}^{*}=\frac{\tau_{\mathrm{ij}}}{\mathrm{HG}} \\
& \Gamma=\frac{\mu_{\mathrm{s}}}{\mathrm{HG}} ; \theta=\frac{\chi}{\mathrm{HG}} .
\end{aligned}
$$

where $\Gamma$ is the rigidity parameter, and $\theta$ is the dimensionless Lame's constant, $\chi$. Having dropped asterisks above dimensionless parameters for convenience, in dimensionless form the equations governing the poroelastic zone become:

$$
\begin{aligned}
& \nabla \cdot\left(\varphi_{\mathrm{f}} \mathbf{V}_{\mathrm{f}}+\varphi_{\mathrm{s}} \mathbf{V}_{\mathrm{s}}\right)=0 \\
& \varphi_{\mathrm{f}} \operatorname{Re}_{\mathrm{f}}\left(\frac{\partial \mathbf{V}_{\mathrm{f}}}{\partial \mathrm{t}}+\left(\mathbf{V}_{\mathrm{f}} \cdot \nabla\right) \mathbf{V}_{\mathrm{f}}\right)=-\varphi_{\mathrm{f}} \nabla \mathrm{p}_{\mathrm{avg}}+\nabla \cdot \tau_{\mathrm{f}}+\zeta\left(\mathbf{V}_{\mathrm{s}}-\mathbf{V}_{\mathrm{f}}\right) \\
& \varphi_{\mathrm{s}} \operatorname{Re}_{\mathrm{s}}\left(\frac{\partial \mathbf{V}_{\mathrm{s}}}{\partial \mathrm{t}}+\left(\mathbf{V}_{\mathrm{s}} \cdot \nabla\right) \mathbf{V}_{\mathrm{s}}\right)=-\varphi_{\mathrm{s}} \nabla \mathrm{p}_{\mathrm{avg}}+\nabla \cdot \tau_{\mathrm{s}}-\zeta\left(\mathbf{V}_{\mathrm{s}}-\mathbf{V}_{\mathrm{f}}\right)
\end{aligned}
$$


$\tau_{\mathrm{f}}=\mathrm{K}_{\mathrm{v}}\left(\nabla \mathbf{V}_{\mathrm{f}}+\nabla \mathbf{V}_{\mathrm{f}}^{\mathrm{T}}\right)$

$\tau_{\mathrm{s}}=\theta \nabla \cdot \mathbf{X}_{\mathrm{s}} \mathbf{I}+\Gamma\left(\nabla \mathbf{X}_{\mathrm{s}}+\nabla \mathbf{X}_{\mathrm{s}}^{\mathrm{T}}\right)$

where we have: $\zeta=\varphi_{\mathrm{f}} \mathrm{K}_{\mathrm{v}} /(\mathrm{h} \kappa)^{2}$ with $\kappa=\sqrt{\mathrm{K}} / \mathrm{R}$ being the permeability parameter, $\mathrm{K}_{\mathrm{v}}=\mu_{\mathrm{f}} / \mu_{\infty}$ is the cross-viscosity ratio, $\operatorname{Re}_{\mathrm{f}}=\rho_{\mathrm{f}}\left(\mathrm{GH}^{3} / \mu_{\infty}{ }^{2}\right)$ is the fluid-phase Reynolds number, $\operatorname{Re}_{\mathrm{s}}=\rho_{\mathrm{S}}\left(\mathrm{GH}^{3} / \mu_{\infty}{ }^{2}\right)$ is the solid-phase Reynolds number, and $\operatorname{Re}=\rho\left(\mathrm{GH}^{3} / \mu_{\infty}{ }^{2}\right)$ is the Reynolds number for the main channel. In dimensionless form, the continuity equation retains its original form whereas the momentum equation becomes:

$$
\operatorname{Re} \frac{\mathrm{DU}}{\mathrm{Dt}}=-\nabla \mathrm{p}+\nabla \cdot \tau
$$

where we have:

$\tau=\eta(\mathrm{S}, \dot{\gamma}) 2 \mathbf{D}$,

$\frac{1}{\Theta}\left(\frac{\mathrm{DS}}{\mathrm{Dt}}\right)=-\mathrm{Tx} \dot{\gamma} \mathrm{S}+(1-\mathrm{S})$

$\eta(S)=1+\varepsilon S$,

where $\Theta=\frac{a}{H G / \mu_{\infty}}$ is the dimensionless rebuild parameter " $a$ ", and $T x=\frac{b / a}{\left(\mu_{\infty} / H G\right)}$ is the dimensionless characteristic time, b/a. As mentioned above, the b/a ratio plays a key role in determining the severity of the fluid's thixotropic behavior. For this reason, we have decided to thoroughly investigate the effect of the b/a ratio on the instability picture, and this amounts to varying $\mathrm{Tx}$ (say, for a given $\mu_{\infty}, \mathrm{H}$, and G). For curiosity reasons, however, we also intend to present limited data as to the effect of the rebuild parameter, $\Theta$, on the critical Reynolds number (say, for a given Tx ). All in all, our FSI problem is governed by the following dimensionless parameters: the main flow Reynolds number $(\mathrm{Re})$, the porous layer's fluid Reynolds number $\left(\operatorname{Re}_{\mathrm{f}}\right)$, the porous layer's solid Reynolds number $\left(\operatorname{Re}_{\mathrm{s}}\right)$, the thixotropy parameter $(\mathrm{Tx})$, the viscosity-gap ratio $(\varepsilon)$, the cross-viscosity ratio $\left(\mathrm{K}_{\mathrm{v}}\right)$, the permeability parameter $(\kappa)$, the flexibility parameter $\left(\Gamma^{-1}\right)$, the pressure gradient parameter $(\Pi)$, the porosity $(\phi)$, the density ratio of the fluid in the porous layer $\left(\Lambda_{\mathrm{f}}\right)$, the density ratio of the solid skeleton in the porous layer $\left(\Lambda_{\mathrm{s}}\right)$, and the rebuild parameter, $\Theta$. For convenience, the definition of each of these dimensionless parameters is listed below:

$$
\begin{aligned}
& \operatorname{Re}_{\mathrm{f}}=\rho_{\mathrm{f}}\left(\mathrm{GH}^{3} / \mu_{\infty}{ }^{2}\right)=\left(\rho_{\mathrm{f}} / \rho\right) \operatorname{Re} \\
& \operatorname{Re}_{\mathrm{s}}=\rho_{\mathrm{s}}\left(\mathrm{GH}^{3} / \mu_{\infty}{ }^{2}\right)=\left(\rho_{\mathrm{s}} / \rho\right) \operatorname{Re} \\
& \operatorname{Re}=\rho\left(\mathrm{GH}^{3} / \mu_{\infty}{ }^{2}\right)
\end{aligned}
$$




$$
\begin{aligned}
& \text { Tx }=\frac{\mathrm{b} / \mathrm{a}}{\mu_{\infty} / \mathrm{HG}} \\
& \Theta=\frac{\mathrm{a}}{\mathrm{HG} / \mu_{\infty}} \\
& \varepsilon=\left(\mu_{0}-\mu_{\infty}\right) / \mu_{\infty} \\
& \mathrm{K}_{\mathrm{v}}=\mu_{\mathrm{f}} / \mu_{\infty} \\
& \kappa=\sqrt{\mathrm{K}} / \mathrm{R} \\
& \Gamma^{-1}=\frac{\mathrm{HG}}{\mu_{\mathrm{s}}} \\
& \Pi=\frac{\mathrm{G}}{(\chi / \mathrm{H})} \\
& \Lambda_{\mathrm{f}}=\rho_{\mathrm{f}} / \rho, \Lambda_{\mathrm{s}}=\rho_{\mathrm{s}} / \rho \\
& \phi=\varphi_{\mathrm{f}}
\end{aligned}
$$

The number of parameters involved in the problem are obviously too many, and some of them are not as important us. For example, $\Lambda_{\mathrm{f}}$ is often realized to be nearly equal to 1 so that $\operatorname{Re}_{\mathrm{f}}=\operatorname{Re}$. For a given channel $(\mathrm{H})$ and a given driving force $(\mathrm{G})$, parameters controlling the performance of poroelastic layers are: $\kappa, \phi, \Gamma^{-1}, \Lambda_{\mathrm{s}}$, and $\mathrm{h}$. Other pertinent parameters are the rheological properties of the two fluids flowing in different zones of the channel (i.e., $\varepsilon, \mathrm{K}_{\mathrm{v}}, \mathrm{Tx}$, and $\Theta$ ). So, for the work to be as comprehensive as possible, we feel obliged to investigate the effects of these parameters on the instability picture. As the first step, we need the basic solution in the main channel and in the poroelastic layer.

\section{Basic Solution}

In the base state (i.e., when there is no disturbance imposed on the interface) the interface is flat. Also, the flow in the channel is steady, fully-developed, and laminar. After some mathematical manipulation (see Appendix C) the dimensionless equations governing the basic solution for the main channel and the porous solid are obtained as [33]:

$$
\begin{aligned}
& \left(1+\frac{\varepsilon}{1+T x|d u / d y|}\right) \frac{d u}{d y}-\Pi=0, \\
& K_{v} \frac{d^{2} u_{f}}{d y^{2}}-\zeta u_{f}-\varphi_{f} \Pi=0,
\end{aligned}
$$




$$
\begin{aligned}
& \Gamma \frac{\mathrm{d}^{2} X_{\mathrm{x}}}{\mathrm{dy}^{2}}+\zeta \mathrm{u}_{\mathrm{f}}-\varphi_{\mathrm{s}} \Pi=0, \\
& \mathrm{v}_{\mathrm{f}}=0, \mathrm{X}_{\mathrm{y}}=\mathrm{y}, \\
& \mathrm{P}_{\mathrm{f}}=\mathrm{P}_{\text {avg }}=\Pi \mathrm{x}+\mathrm{c}_{0} .
\end{aligned}
$$

In Eq. 28, $\mathrm{c}_{0}$ is the constant of integration which can arbitrarily be set equal to zero. The boundary conditions corresponding to the basic solution are:

$$
\left\{\begin{array}{l}
\left.\mathrm{u}_{\mathrm{f}}\right|_{\mathrm{y}=-\mathrm{h}}=0=\left.\mathrm{X}_{\mathrm{x}}\right|_{\mathrm{y}=-\mathrm{h}} \\
\left.\mathrm{u}\right|_{\mathrm{y}=1}=0 \\
\left.\varphi_{\mathrm{f}} \Lambda_{\mathrm{f}} \mathrm{u}_{\mathrm{f}}\right|_{\mathrm{y}=0}=\left.\mathrm{u}\right|_{\mathrm{y}=0} \\
\left.\tau_{\mathrm{xy}, \mathrm{f}}\right|_{\mathrm{y}=0}=\left.\varphi_{\mathrm{f}} \tau_{\mathrm{xy}}\right|_{\mathrm{y}=0} \\
\left.\tau_{\mathrm{xy}, \mathrm{s}}\right|_{\mathrm{y}=0}=\left.\varphi_{\mathrm{s}} \tau_{\mathrm{xy}}\right|_{\mathrm{y}=0}
\end{array}\right.
$$

There is no analytical solution for the basic solution, and so we look for a numerical solution (see Appendix C). Having found the basic solution, which is by itself a formidable task, the basic stress tensors for the solid and fluid phases in the poroelastic layer can be obtained as:

$$
\tau_{\mathrm{s}}=\Gamma\left[\begin{array}{cc}
0 & \frac{\mathrm{dX}}{\mathrm{dy}} \\
\frac{\mathrm{dX}}{\mathrm{dy}} & 0
\end{array}\right] ; \tau_{\mathrm{f}}=\mathrm{K}_{\mathrm{v}}\left[\begin{array}{cc}
0 & \frac{\mathrm{du}}{\mathrm{dy}} \\
\frac{\mathrm{du_{f }}}{\mathrm{dy}} & 0
\end{array}\right] .
$$

In the next section, we slightly perturb the above basic solutions and see if there is any mode which can be excited to become eventually unstable.

\section{Linear Stability Analysis}

Having found the basic velocity field in the main channel and the basic flow/deformation in the poroelastic region (which consists of the deformation field for the solid phase and the velocity field for the fluid phase) we subject them to infinitesimally-small perturbations and see how they evolve in the course of time [45]. For simplicity, we assume that the perturbations are two-dimensional. To that end, based on the concept of the normal-mode analysis, the perturbed parameters for the poroelastic and core regions are written as:

$$
\begin{aligned}
& \mathrm{u}(\mathrm{x}, \mathrm{y}, \mathrm{t})=\overline{\mathrm{u}}(\mathrm{x}, \mathrm{y}, \mathrm{t})+\mathrm{u}^{\prime}(\mathrm{x}, \mathrm{y}, \mathrm{t})=\overline{\mathrm{u}}(\mathrm{y})+\hat{\mathrm{u}}(\mathrm{y}) \mathrm{e}^{\mathrm{i} \alpha \mathrm{x}+\omega \mathrm{t}} \\
& \mathrm{v}(\mathrm{x}, \mathrm{y}, \mathrm{t})=\overline{\mathrm{v}}(\mathrm{x}, \mathrm{y}, \mathrm{t})+\mathrm{v}^{\prime}(\mathrm{x}, \mathrm{y}, \mathrm{t})=0+\hat{\mathrm{v}}(\mathrm{y}) \mathrm{e}^{\mathrm{i} \alpha \mathrm{x}+\omega \mathrm{t}}
\end{aligned}
$$




$$
\begin{aligned}
& S(x, y, t)=\bar{S}(x, y, t)+S^{\prime}(x, y, t)=\bar{S}(y)+\hat{S}(y) e^{i \alpha x+\omega t} \\
& p(x, y, t)=\bar{p}(x, y, t)+p^{\prime}(x, y, t)=\Pi x+\hat{p}(y) e^{i \alpha x+\omega t} \\
& u_{f}(x, y, t)=\bar{u}_{f}(x, y, t)+u_{f}^{\prime}(x, y, t)=\bar{u}_{f}(y)+\hat{u}_{f}(y) e^{i \alpha x+\omega t} \\
& v_{f}(x, y, t)=\bar{v}_{f}(x, y, t)+v_{f}^{\prime}(x, y, t)=0+\hat{v}_{f}(y) e^{i \alpha x+\omega t} \\
& X_{x}(x, y, t)=\bar{X}_{x}(x, y, t)+X_{x}^{\prime}(x, y, t)=x+\bar{X}_{x}(y)+\hat{X}_{x}(y) e^{i \alpha x+\omega t} \\
& X_{y}(x, y, t)=\bar{X}_{y}(x, y, t)+X_{y}^{\prime}(x, y, t)=y+\hat{X}_{y}(y) e^{i \alpha x+\omega t} \\
& p_{\text {avg }}(x, y, t)=\bar{p}_{\text {avg }}(x, y, t)+p_{\text {avg }}^{\prime}(x, y, t)=\Pi x+\hat{p}_{a v g}(y) e^{i \alpha x+\omega t}
\end{aligned}
$$

where $\mathrm{u}, \mathrm{v}, \mathrm{p}$ are the velocity and pressure fields for the thixotropic fluid in the main channel, $\mathrm{u}_{\mathrm{f}}, \mathrm{v}_{\mathrm{f}}$ are the velocity components for the fluid phase inside the poroelastic layer, and $\mathrm{X}_{\mathrm{x}}, \mathrm{X}_{\mathrm{y}}$ are the displacement components for the solid matrix in this layer. In these equations the overbar (-) means the basic flow, prime (') means perturbations, and hat $\left({ }^{\wedge}\right)$ means the amplitude of the eigenfunctions. Moreover, $\alpha$ is the wavenumber and $\omega$ is the growth rate. In temporal stability analysis used in the present work, the wavenumber is a real number whereas the growth rate is allowed to be a complex number. The system is labeled unstable provided that there exists a wavenumber for which the real part of $\omega$ is positive. To determine such wavenumbers, we proceed with inserting the perturbed quantities into the time-dependent equations of motion for the solid and fluid phases in order to obtain the differential equations governing the time-evolution of the perturbations. After some tedious mathematical manipulations [see Ref. 33 for the details], we end up with the following stability equations for the poroelastic layer:

$$
\varphi_{\mathrm{f}} \mathrm{i} \alpha \hat{\mathrm{u}}_{\mathrm{f}}(\mathrm{y})+\omega i \alpha \varphi_{\mathrm{s}} \hat{\mathrm{X}}_{\mathrm{x}}(\mathrm{y})+\varphi_{\mathrm{f}} \frac{\mathrm{d} \hat{\mathrm{v}}_{\mathrm{f}}}{\mathrm{dy}}+\varphi_{\mathrm{s}} \omega \frac{\mathrm{d} \hat{\mathrm{X}}_{\mathrm{y}}}{\mathrm{dy}}=0
$$

$\varphi_{\mathrm{f}} \operatorname{Re}_{\mathrm{f}} \hat{\mathrm{u}}_{\mathrm{f}}(\mathrm{y})\left[\omega+\mathrm{i} \alpha \mathrm{u}_{\mathrm{f}}(\mathrm{y})\right]+\varphi_{\mathrm{f}} \operatorname{Re}_{\mathrm{f}} \hat{\mathrm{v}}_{\mathrm{f}}(\mathrm{y}) \frac{\mathrm{du}}{\mathrm{dy}}+\mathrm{i} \alpha \varphi_{\mathrm{f}} \hat{\mathrm{p}}_{\mathrm{avg}}(\mathrm{y})+$

$K_{v}\left(2 \alpha^{2} \hat{u}_{f}(y)-\frac{d^{2} \hat{u}_{f}}{d y^{2}}-i \alpha \frac{d \hat{v}_{f}}{d y}\right)-\zeta\left[\omega \hat{X}_{x}(y)-\hat{u}_{f}(y)\right]=0$,

$\varphi_{\mathrm{f}} \operatorname{Re}_{\mathrm{f}} \hat{\mathrm{v}}_{\mathrm{f}}(\mathrm{y})\left[\omega+\mathrm{i} \alpha \mathrm{u}_{\mathrm{f}}(\mathrm{y})\right]+\varphi_{\mathrm{f}} \frac{\mathrm{d} \hat{\mathrm{p}}_{\mathrm{avg}}}{\mathrm{dy}}-\mathrm{K}_{\mathrm{v}}\left(2 \frac{\mathrm{d}^{2} \hat{\mathrm{v}}_{\mathrm{f}}}{\mathrm{dy}^{2}}+\mathrm{i} \alpha \frac{\mathrm{d} \hat{\mathrm{u}}_{\mathrm{f}}}{\mathrm{dy}}-\alpha^{2} \hat{\mathrm{v}}_{\mathrm{f}}(\mathrm{y})\right)-$

$\zeta\left[\omega \hat{X}_{y}(y)-\hat{v}_{f}(y)\right]=0$, 
$\operatorname{Re}_{\mathrm{s}} \omega^{2} \varphi_{\mathrm{s}} \hat{\mathrm{X}}_{\mathrm{x}}(\mathrm{y})+\mathrm{i} \alpha \varphi_{\mathrm{s}} \hat{\mathrm{p}}_{\mathrm{avg}}(\mathrm{y})-\mathrm{i} \alpha \theta\left(\mathrm{i} \alpha \hat{\mathrm{X}}_{\mathrm{x}}(\mathrm{y})+\frac{\mathrm{d} \hat{\mathrm{X}}_{\mathrm{y}}}{\mathrm{dy}}\right)+$
$2 \Gamma \alpha^{2} \hat{\mathrm{X}}_{\mathrm{x}}(\mathrm{y})-\Gamma\left(\frac{\mathrm{d}^{2} \hat{\mathrm{X}}_{\mathrm{x}}}{\mathrm{dy}^{2}}+\mathrm{i} \alpha \frac{\mathrm{d} \hat{\mathrm{X}}_{\mathrm{y}}}{\mathrm{dy}}\right)+\zeta\left(\omega \hat{\mathrm{X}}_{\mathrm{x}}(\mathrm{y})-\hat{\mathrm{u}}_{\mathrm{f}}(\mathrm{y})\right)=0$

$\operatorname{Re}_{\mathrm{s}} \omega^{2} \varphi_{\mathrm{s}} \hat{\mathrm{X}}_{\mathrm{y}}(\mathrm{y})+\varphi_{\mathrm{s}} \frac{\mathrm{d} \hat{\mathrm{p}}_{\mathrm{avg}}}{\mathrm{dy}}-\mathrm{i} \alpha \theta \frac{\mathrm{d} \hat{\mathrm{X}}_{\mathrm{x}}}{\mathrm{dy}}-(\theta+2 \Gamma) \frac{\mathrm{d}^{2} \hat{\mathrm{X}}_{\mathrm{y}}}{\mathrm{dy}^{2}}-$

$\Gamma i \alpha \frac{d \hat{X}_{x}}{d y}+\Gamma \alpha^{2} \hat{X}_{y}(y)+\zeta\left(\omega \hat{X}_{y}(y)-\hat{v}_{f}(y)\right)=0$.

As to the stability equations for the Moore fluid flowing in the main channel the following system of equations are obtained for the eigenfunction $\hat{v}$; see Ref. 33:

$$
\begin{aligned}
& a_{1}(y) \frac{d^{4} \hat{v}}{d y^{4}}+a_{2}(y) \frac{d^{3} \hat{v}}{d y^{3}}+a_{3}(y) \frac{d^{2} \hat{v}}{d y^{2}}+a_{4}(y) \frac{d \hat{v}}{d y}+a_{5}(y) \hat{v}+a_{6}(y) \frac{d^{2} \hat{S}}{d y^{2}}+a_{7}(y) \frac{d \hat{S}}{d y}+a_{8}(y) \hat{S}=0 \\
& b_{1}(y) \frac{d^{2} \hat{v}}{d y^{2}}+b_{2}(y) \hat{v}+b_{3}(y) \hat{S}=0
\end{aligned}
$$

where the coefficients are:

$$
\begin{aligned}
& a_{1}(y)=\frac{1}{\alpha^{2}(1+\varepsilon \bar{S}(y))^{2}}, \\
& \mathrm{a}_{2}(\mathrm{y})=-\frac{4 \varepsilon}{\alpha^{2}(1+\varepsilon \overline{\mathrm{S}})^{3}}\left(\frac{\mathrm{d} \overline{\mathrm{S}}}{\mathrm{dy}}\right), \\
& \mathrm{a}_{3}(\mathrm{y})=\frac{6 \varepsilon^{2}}{\alpha^{2}(1+\varepsilon \overline{\mathrm{S}}(\mathrm{y}))^{4}}\left(\frac{\mathrm{d} \overline{\mathrm{S}}}{\mathrm{dy}}\right)^{2}-\frac{\mathrm{Re}}{\alpha^{2}}(\omega+\mathrm{i} \alpha \overline{\mathrm{u}}(\mathrm{y}))-\frac{2 \varepsilon}{\alpha^{2}(1+\varepsilon \overline{\mathrm{S}}(\mathrm{y}))^{3}}\left(\frac{\mathrm{d}^{2} \overline{\mathrm{S}}}{\mathrm{dy}^{2}}\right)-\frac{2}{(1+\varepsilon \overline{\mathrm{S}}(\mathrm{y}))^{3}}, \\
& \mathrm{a}_{4}(\mathrm{y})=\frac{4 \varepsilon}{(1+\varepsilon \overline{\mathrm{S}}(\mathrm{y}))^{3}}\left(\frac{\mathrm{d} \overline{\mathrm{S}}}{\mathrm{dy}}\right) \\
& \mathrm{a}_{5}=\frac{\alpha^{2}}{(1+\varepsilon \overline{\mathrm{S}}(\mathrm{y}))^{2}}+\operatorname{Re}\left(+\mathrm{i} \alpha \overline{\mathrm{u}}(\mathrm{y})-\frac{1}{\mathrm{i} \alpha}\left(\frac{\mathrm{d} \overline{\mathrm{u}}}{\mathrm{dy}}\right)+\omega\right)+\frac{6 \varepsilon^{2}}{(1+\varepsilon \overline{\mathrm{S}}(\mathrm{y}))^{4}}\left(\frac{\mathrm{d} \overline{\mathrm{S}}}{\mathrm{dy}}\right)^{2}-\frac{2 \varepsilon}{(1+\varepsilon \overline{\mathrm{S}}(\mathrm{y}))^{3}}\left(\frac{\mathrm{d}^{2} \overline{\mathrm{S}}}{\mathrm{dy}^{2}}\right), \\
& \mathrm{a}_{6}(\mathrm{y})=\frac{2 \mathrm{i}}{\alpha} \frac{\varepsilon}{(1+\varepsilon \overline{\mathrm{S}}(\mathrm{y}))^{3}}\left(\frac{\mathrm{d} \overline{\mathrm{u}}}{\mathrm{dy}}\right) \\
& \mathrm{a}_{7}(\mathrm{y})=-\frac{12 \mathrm{i}}{\alpha} \frac{\varepsilon^{2}}{(1+\varepsilon \overline{\mathrm{S}}(\mathrm{y}))^{4}}\left(\frac{\mathrm{d} \overline{\mathrm{u}}}{\mathrm{dy}}\right)\left(\frac{\mathrm{d} \overline{\mathrm{S}}}{\mathrm{dy}}\right)+\frac{4 \mathrm{i}}{\alpha} \frac{\varepsilon}{(1+\varepsilon \overline{\mathrm{S}}(\mathrm{y}))^{3}}\left(\frac{\mathrm{d}^{2} \overline{\mathrm{u}}}{\mathrm{dy}^{2}}\right),
\end{aligned}
$$




$$
\begin{aligned}
& \mathrm{a}_{8}(\mathrm{y})=\frac{24 \mathrm{i}}{\alpha} \frac{\varepsilon^{3}}{(1+\varepsilon \overline{\mathrm{S}}(\mathrm{y}))^{5}}\left(\frac{\mathrm{d} \overline{\mathrm{u}}}{\mathrm{dy}}\right)\left(\frac{\mathrm{d} \overline{\mathrm{S}}}{\mathrm{dy}}\right)-\frac{6 \mathrm{i}}{\alpha} \frac{\varepsilon^{2}}{(1+\varepsilon \overline{\mathrm{S}}(\mathrm{y}))^{4}}\left(\frac{\mathrm{d} \overline{\mathrm{u}}}{\mathrm{dy}}\right)\left(\frac{\mathrm{d}^{2} \overline{\mathrm{S}}}{\mathrm{dy}^{2}}\right)- \\
& \frac{12 \mathrm{i}}{\alpha} \frac{\varepsilon^{2}}{(1+\varepsilon \overline{\mathrm{S}}(\mathrm{y}))^{4}}\left(\frac{\mathrm{d}^{2} \overline{\mathrm{u}}}{\mathrm{dy} \mathrm{y}^{2}}\right)\left(\frac{\mathrm{d} \overline{\mathrm{S}}}{\mathrm{dy}}\right)+\frac{2 \mathrm{i}}{\alpha} \frac{\varepsilon}{(1+\varepsilon \overline{\mathrm{S}}(\mathrm{y}))^{3}}\left(\frac{\mathrm{d}^{3} \overline{\mathrm{u}}}{d y^{3}}+\alpha^{2} \frac{\mathrm{d} \overline{\mathrm{u}}}{\mathrm{dy}}\right), \\
& \mathrm{b}_{1}(\mathrm{y})=-\operatorname{sgn}\left(\left|\frac{\mathrm{d} \overline{\mathrm{u}}}{\mathrm{dy}}\right|\right) \frac{\mathrm{Tx}}{\mathrm{i} \alpha} \overline{\mathrm{S}}(\mathrm{y}) \\
& \mathrm{b}_{2}(\mathrm{y})=\frac{1}{\Theta} \frac{\mathrm{d} \overline{\mathrm{S}}}{\mathrm{dy}}+\mathrm{i} \alpha \mathrm{T} \mathrm{x} \operatorname{sgn}\left(\left|\frac{\mathrm{d} \overline{\mathrm{u}}}{\mathrm{dy}}\right|\right) \overline{\mathrm{S}}(\mathrm{y}) \\
& \mathrm{b}_{3}(\mathrm{y})=1+\frac{\omega}{\Theta}+\frac{\mathrm{i} \alpha}{\Theta} \overline{\mathrm{u}}(\mathrm{y})+\mathrm{Tx}\left|\frac{\mathrm{d} \overline{\mathrm{u}}}{\mathrm{dy}}\right|,
\end{aligned}
$$

where "sgn" stands for the sign function. As to the boundary conditions needed for solving the instability equations, we rely on the no-slip and no-penetration conditions for the core flow at the upper wall; that is:

$$
\begin{aligned}
& \left.\hat{\mathrm{v}}\right|_{y=1}=0 \\
& \left.\hat{\mathrm{u}}\right|_{\mathrm{y}=1}=\left.0 \stackrel{\text { Continuty }}{\Rightarrow} \frac{\mathrm{d} \hat{\mathrm{v}}}{\mathrm{dy}}\right|_{\mathrm{y}=1}=0
\end{aligned}
$$

In the poroelastic region, no-slip/no-penetration are used as the boundary conditions for the fluid phase. On the surface of the lower rigid plate, i.e., where the poroelastic material is fixed, the displacement of the solid phase is forced to be zero. Thus, we have:

$$
\begin{aligned}
& \left.\hat{u}_{\mathrm{f}}\right|_{\mathrm{y}=-\mathrm{h}}=0=\left.\hat{\mathrm{v}}_{\mathrm{f}}\right|_{\mathrm{y}=-\mathrm{h}} \\
& \left.\hat{\mathrm{X}}_{\mathrm{x}}\right|_{\mathrm{y}=-\mathrm{h}}=0=\left.\hat{\mathrm{X}}_{\mathrm{y}}\right|_{\mathrm{y}=-\mathrm{h}}
\end{aligned}
$$

To determine the boundary-coupled conditions at the fluid/poroelastic-layer interface, we rely on the Taylor expansion for all Eulerian variables. Continuity of the tangential and normal velocities together with the continuity of the shear and normal stresses for each phase in the poroelastic region can provide us with six more boundary conditions at this location. To that end, the interface is shifted to a new position after each perturbation. First-order Taylor expansion is then used for all Eulerian variables so that we can write,

$$
\begin{aligned}
& \left.\Phi\right|_{\text {perturbed interface }}=\left.\Psi\right|_{\text {perturbed interface }} \\
& \left.\Phi\right|_{\mathrm{y}=0}+\xi\left(\left.\frac{\partial \Phi}{\partial \mathrm{x}}\right|_{\mathrm{y}=0}\right)+\delta\left(\left.\frac{\partial \Phi}{\partial \mathrm{y}}\right|_{\mathrm{y}=0}\right)=\left.\Psi\right|_{\text {perturbed interface }}
\end{aligned}
$$

where $\Phi$ refers to any variable for the main fluid and $\Psi$ to its corresponding variable in the poroelastic layer at each side of the interface. In the above relationships, $\xi$ and $\delta$ are, respectively, the amplitudes of 
the horizontal and vertical components of the perturbed interface at $y=0$. By imposing the kinematic boundary condition at the interface, it turns out that $\xi$ and $\delta$ must satisfy the following transport equations [33]:

$$
\begin{aligned}
& \frac{\mathrm{D} \delta}{\mathrm{Dt}}=\frac{\partial \delta}{\partial \mathrm{t}}+\left.\mathrm{u}\right|_{\mathrm{x}=\xi, \mathrm{y}=\delta}\left(\frac{\partial \delta}{\partial \mathrm{x}}\right)=\left.\mathrm{v}\right|_{\mathrm{x}=\xi, \mathrm{y}=\delta} \\
& \frac{\mathrm{D} \xi}{\mathrm{Dt}}=\frac{\partial \xi}{\partial \mathrm{t}}+\left.\mathrm{u}\right|_{\mathrm{x}=\xi, \mathrm{y}=\delta}\left(\frac{\partial \xi}{\partial \mathrm{x}}\right)=\left.\mathrm{u}\right|_{\mathrm{x}=\xi, \mathrm{y}=\delta}
\end{aligned}
$$

The above equations can be linearized as:

$$
\begin{aligned}
& \frac{\partial \delta}{\partial \mathrm{t}}+\left.\overline{\mathrm{u}}\right|_{\mathrm{y}=0}\left(\frac{\partial \delta}{\partial \mathrm{x}}\right)=\left.\mathrm{v}^{\prime}\right|_{\mathrm{y}=0} \\
& \frac{\partial \xi}{\partial \mathrm{t}}+\left.\overline{\mathrm{u}}\right|_{\mathrm{y}=0}\left(\frac{\partial \xi}{\partial \mathrm{x}}\right)=\left.\mathrm{u}^{\prime}\right|_{\mathrm{y}=0}+\left.\delta \frac{\mathrm{d} \overline{\mathrm{u}}}{\mathrm{dy}}\right|_{\mathrm{y}=0}
\end{aligned}
$$

At $y=0$, the linearized form of the six boundary conditions mentioned above (i.e., the continuity of the normal/tangential velocities, the continuity of normal/tangential stresses for the fluid phase, and also the continuity of the normal/tangential stresses for the solid phase) are then obtained as:

$$
\begin{aligned}
& \left.\hat{\mathrm{u}}\right|_{\mathrm{y}=0}+\left.\frac{\mathrm{d} \overline{\mathrm{u}}}{\mathrm{dy}}\right|_{\mathrm{y}=0} \delta-\varphi_{\mathrm{s}} \Lambda_{\mathrm{s}} \omega \hat{\mathrm{X}}_{\mathrm{x}}-\varphi_{\mathrm{f}} \Lambda_{\mathrm{f}}\left(\left.\hat{\mathrm{u}}_{\mathrm{f}}\right|_{\mathrm{y}=0}+\left.\frac{\mathrm{d} \overline{\mathrm{u}}_{\mathrm{f}}}{\mathrm{dy}}\right|_{\mathrm{y}=0} \delta\right)=0 \\
& \left.\hat{\mathrm{v}}\right|_{\mathrm{y}=0}-\varphi_{\mathrm{s}} \Lambda_{\mathrm{s}} \omega \hat{\mathrm{X}}_{\mathrm{y}}-\left.\varphi_{\mathrm{f}} \Lambda_{\mathrm{f}} \hat{\mathrm{v}}_{\mathrm{f}}\right|_{\mathrm{y}=0}=0 \\
& \left.\varphi_{\mathrm{s}} \hat{\tau}_{\mathrm{xy}}\right|_{\mathrm{y}=0}-\left.\hat{\tau}_{\mathrm{xy}, \mathrm{s}}\right|_{\mathrm{y}=0}+\left(\left.\varphi_{\mathrm{s}} \frac{\mathrm{d} \bar{\tau}_{\mathrm{xy}}}{\mathrm{dy}}\right|_{\mathrm{y}=0}-\left.\frac{\mathrm{d} \bar{\tau}_{\mathrm{xy}, \mathrm{s}}}{\mathrm{dy}}\right|_{\mathrm{y}=0}\right) \delta=0 \\
& \varphi_{s}\left(\hat{p}_{a v g}-\hat{p}+\left.\hat{\tau}_{y y}\right|_{y=0}\right)-\left.\hat{\tau}_{y y, s}\right|_{y=0}+i \alpha\left(\left.\bar{\tau}_{x y, s}\right|_{y=0}-\left.\varphi_{s} \bar{\tau}_{x y}\right|_{y=0}\right) \delta=0 \\
& \left.\varphi_{\mathrm{f}} \hat{\tau}_{\mathrm{xy}}\right|_{\mathrm{y}=0}-\left.\hat{\tau}_{\mathrm{xy}, \mathrm{f}}\right|_{\mathrm{y}=0}+\left(\left.\varphi_{\mathrm{f}} \frac{\mathrm{d} \bar{\tau}_{\mathrm{xy}}}{\mathrm{dy}}\right|_{y=0}-\left.\frac{\mathrm{d} \bar{\tau}_{\mathrm{xy}, \mathrm{f}}}{\mathrm{dy}}\right|_{y=0}\right) \delta=0 \\
& \varphi_{\mathrm{f}}\left(\left.\hat{\mathrm{p}}_{\mathrm{avg}}\right|_{\mathrm{y}=0}-\left.\hat{\mathrm{p}}\right|_{\mathrm{y}=0}+\left.\hat{\tau}_{\mathrm{yy}}\right|_{\mathrm{y}=0}\right)-\left.\hat{\tau}_{\mathrm{yy}, \mathrm{f}}\right|_{\mathrm{y}=0}+\mathrm{i} \alpha\left(\left.\tau_{\mathrm{xy}, \mathrm{f}}\right|_{\mathrm{y}=0}-\left.\varphi_{\mathrm{f}} \tau_{\mathrm{xy}}\right|_{\mathrm{y}=0}\right) \delta=0
\end{aligned}
$$

It should be noted that Eqs. 51 and 52 are the balance of shear and normal stresses between the solid phase in the poroelastic layer and the thixotropic fluid in the main channel at the interface. Similarly, Eqs. 53 and 54 are the balance of shear and normal stresses between the fluid phase in the poroelastic region and the core fluid in the main channel. It is worth mentioning that the horizontal component of the perturbed interface (i.e., $\xi$ ) drops from Eqs. 49-54 and also from Eq. 44 owing to the fully-developed 
nature of the basic state. The perturbed stresses for the fluid/solid phases in the poroelastic layer can then be obtained as:

$$
\begin{gathered}
\hat{\tau}_{f}=K_{v}\left[\begin{array}{cc}
2 i \alpha \hat{u}_{f}(y) & \frac{d \hat{u}_{f}}{d y}+i \alpha \hat{v}_{f}(y) \\
\frac{d \hat{u}_{f}}{d y}+i \alpha \hat{v}_{f}(y) & 2 \frac{d \hat{v}_{f}}{d y}
\end{array}\right] e^{i \alpha x+\omega t} \\
\hat{\tau}_{s}=\left[\begin{array}{cc}
(2 \Gamma+\theta) i \alpha \hat{X}_{x}+\frac{d \hat{X}_{y}}{d y} & \Gamma\left(\frac{d \hat{X}_{x}}{d y}+i \alpha \hat{X}_{y}\right) \\
\Gamma\left(\frac{d \hat{X}_{x}}{d y}+i \alpha \hat{X}_{y}\right) & (2 \Gamma+\theta) \frac{d \hat{X}_{y}}{d y}+i \alpha \hat{X}_{x}
\end{array}\right] e^{i \alpha x+\omega t}
\end{gathered}
$$

Similarly, the perturbed stress tensor for the thixotropic fluid in the main channel becomes:

$$
\hat{\tau}=\left[\begin{array}{c}
2 i \alpha[1+\varepsilon \bar{S}(y)] \hat{u}(y) \\
\left\{\begin{array}{l}
{[1+\varepsilon \bar{S}(y)]\left(\frac{d \hat{u}}{d y}+i \alpha \hat{v}(y)\right)+} \\
\varepsilon \frac{d \bar{u}}{d y} \hat{\lambda}(y)
\end{array}\right\} \\
\varepsilon \frac{d \bar{u}}{d y} \hat{\lambda}(y) \\
2[1+\varepsilon \bar{S}(y)] \frac{d \hat{v}}{d y}
\end{array}\right] e^{i \alpha x+\omega t}
$$

And, this completes our stability equations for the Moore model. In the next section we proceed with solving these equations.

\section{Numerical Method}

Equations 32-37 subject to the boundary conditions given by Eqs. 39-54, can be solved numerically using the spectral method [46]. In this robust numerical method, all variables are expanded as a sum of certain trial functions. The choice of the trial function is arbitrary and depends on the degree of nonlinearity of the problem at hand. In this work, we rely on the Chebyshev polynomials for this purpose. To that end, the velocity, deformation, and pressure in Eqs. 32-37 are expanded as:

$$
\begin{aligned}
& \hat{u}_{f}=\sum_{j=1}^{N} a_{j} T_{j}(Z) ; \hat{v}_{f}=\sum_{j=1}^{N} b_{j} T_{j}(Z) ; \hat{u}=\sum_{J=1}^{M+1} e_{J} T_{J}(Y) ; \hat{v}=\sum_{J=1}^{M+1} f_{J} T_{J}(Y) ; \hat{S}=\sum_{J=1}^{M} g_{J} T_{J}(Y) \\
& \hat{X}_{x}=\sum_{j=1}^{N} c_{j} T_{j}(Z) ; \hat{X}_{y}=\sum_{j=1}^{N} d_{j} T_{j}(Z) ; \hat{p}_{a v g}=\sum_{j=1}^{N} q_{j} T_{j}(Z) ; \hat{p}=\sum_{J=1}^{M+1} h_{J} T_{J}(Y)
\end{aligned}
$$


In the above equations, $\mathrm{Z}=(2 \mathrm{y}+\mathrm{h}) / \mathrm{h}$ and $\mathrm{Y}=2 \mathrm{y}-1$ are used for the poroelastic and core regions, respectively. They are the Chebyshev variables valid for the dimensionless coordinate $\mathrm{y} \in[-\mathrm{h}, 0]$ and $y \in[0,1]$, respectively. These two change-of-variables can map the solution domain in the poroelastic layer and the core region into the dimensionless interval $[-1,1]$ as required. After substituting the perturbed quantities (i.e., Eq. 56) in Eqs. 32-54 and then using the spectral collocation method, a linear system of algebraic equations is obtained in the form of: $\mathbf{A} \Upsilon+\omega \mathbf{B} \Upsilon+\omega^{2} \mathbf{C} \Upsilon=0$, where $\mathbf{A}, \mathbf{B}, \mathbf{C}$ are the coefficient matrices, $\omega$ is the eigenvalue, and $\Upsilon$ is the eigenvector. This nonlinear eigenvalue problem (second-order polynomial with respect to $\omega$ ) can be solved using an iterative method. We rely on the MATLAB's robust polyeig function to capture the eigenvalues. To validate our spectral code, it was used to recover the critical Reynolds number for the classic plane Poiseuille flow of Newtonian fluids in rigid channels (i.e., for $\varepsilon=0, \kappa=0, \Gamma \rightarrow \infty)$. Using the maximum velocity in the core as the characteristic velocity, our code could easily recover the well-known critical Reynolds number of $\operatorname{Re}_{\mathrm{cr}}=5772.22$ and the critical wavenumber of $\alpha_{c r}=1.02$ for this benchmark problem [47]. (Using our definition for the Reynolds number and the length scale used in our work, this classic critical Reynolds number and wavenumber amount to $\operatorname{Re}_{\mathrm{cr}}=9.54 \times 10^{4}$ and $\alpha_{\mathrm{cr}}=2.04$, based on our formulations.) Figure 3 shows the spectrum of the eigenvalues obtained with our code for this benchmark problem using $\mathrm{N}=\mathrm{M}=132$ Chebyshev terms. As can be seen in this figure, our eigenvalue spectrum is quite similar to that reported in Ref. 47, and this is particularly so for the so-called center modes (i.e., the modes on the right branch) and also the wall modes (i.e., the modes on the left branch). On the other hand, the so-called damped modes (i.e., the modes in the middle branch having $\mathrm{C}_{\mathrm{r}}$ roughly equal to 0.67 ) are seen to be somewhat different between the two sets of results. This can be attributed to the notion that we have obtained our results for $\Gamma^{-1}=1.5 \times 10^{-7}$ (which closely represents the rigid case in our simulations) whereas the classic results are for exactly $\Gamma^{-1}=0$. This little exercise reveals that flexibility, no matter how small it is, can dramatically affect the damped modes - a fact to be elaborated more in subsequent sections. This should be contrasted with the effect of porosity which is long established to affect mostly the wall modes. Still, as far as the most dangerous eigenmode is concerned, our prediction for the dangerous mode $\left(C_{r}=0.269\right)$, shown by a green solid circle in Fig. 3, is virtually the same as that reported in Ref. 47.

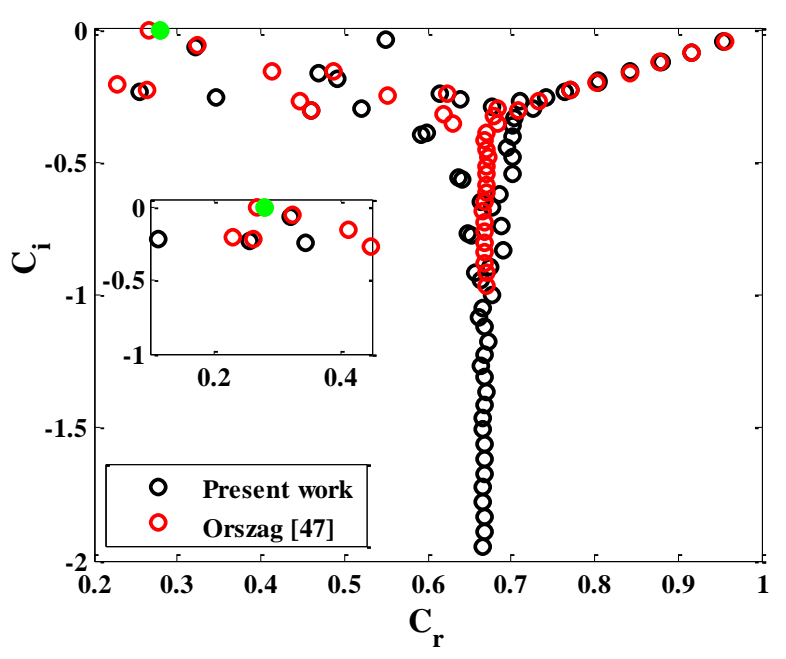


Figure 3: A comparison between the eigenvalue spectrum by our code with published data for the classic (i.e., rigid impermeable) plane Poiseuille flow of Newtonian fluids $\left(\operatorname{Re}_{c r}=5772.22\right)$.

We have also tried to verify our code using stability data available in the literature for the poroelastic case, but unfortunately we could not find such data in the open literature. For the special case where the lining is porous but inelastic, fortunately such data are available for the $\mathrm{N} / \mathrm{N}$ case in a similar channel. Sparrow et al [11] resorted to the linear stability theory in order to investigate the role played by the layer's permeability on the critical Reynolds number using Beaver's slip boundary condition. Their results show that permeability initially stabilizes the flow but its effect becomes destabilizing beyond a threshold value. In the present work, we have not relied on "slip" as a boundary condition. Instead we have resorted to the biphasic theory which be default allows the velocity at the interface to be non-zero in a continuous fashion. Our numerical results (see next section) indeed predicts the existence of a threshold permeability. That is to say that, our results qualitatively confirm Sparrow's prediction [11] that permeability can have a stabilizing or destabilizing effect on the core flow depending on its magnitude being smaller or larger than a threshold value. Although there is no stability data for poroelastic layers in the open literature, fortunately, basic flow profiles are available for the N/N case in such channels. Figure 4 shows a comparison between the fluid-flux profile (Q) obtained in this work with the profile reported by Hou et al [22] using the biphasic theory. As can be seen in this figure, the code is doing an excellent job in reproducing the basic-flow velocity profiles in the main channel and also in the poroelastic layer. It needs to be mentioned that to ensure that the results are independent of the number of Chebyshev polynomials, we have relied on 51 nodes for the main channel and 86 nodes for the poroelastic layer to obtain these results. This number of nodes have also been realized to be good enough for the Moore/Newtonian and Quemada/Newtonian simulations as far as basic flow/deformation is concerned; for obtaining the neutral stability curve, more Chebyshev terms were needed.

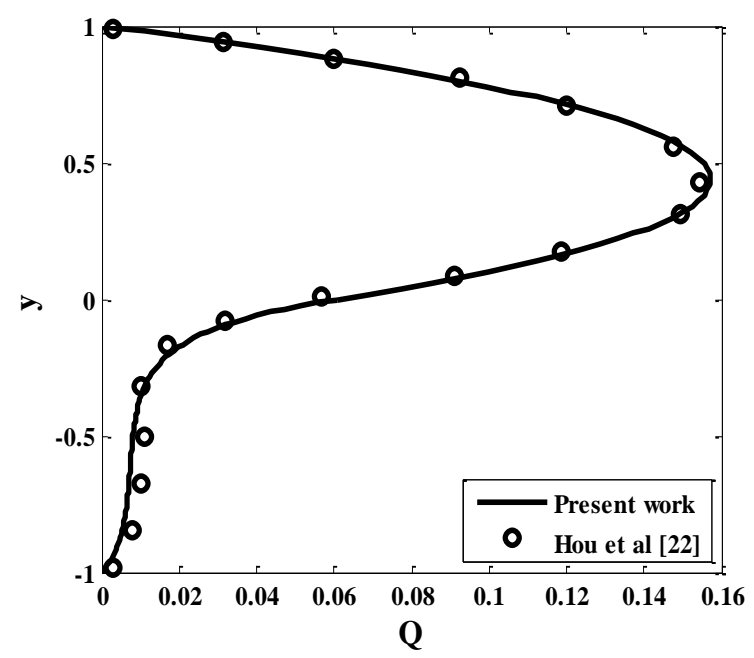

Figure 4: Verification of the basic flow flux for the N/N case for the poroelastic channel shown schematically in Fig. $1\left(\mathrm{~K}_{\mathrm{v}}=1, \phi=0.5, \kappa=0.0857\right)$.

\section{Results and Discussions}


Having verified the code, we are now ready to present our stability results obtained for the poroelastic channel depicted schematically in Fig. 1. We start with presenting the basic flow velocity and deformation fields for the Newtonian/Newtonian (N/N) case. We then proceed with obtaining results for the Moore/Newtonian $(\mathrm{M} / \mathrm{N})$ case; limited results have also been obtained for the Quemada/Newtonian $(\mathrm{Q} / \mathrm{N})$ case which are presented in Appendix D. Our main objective is to figure out the role played by the permeability, porosity, and elasticity of the poroelastic layer in stabilizing/destabilizing the core flow. For the $\mathrm{M} / \mathrm{N}$ and $\mathrm{Q} / \mathrm{N}$ cases, we are also interested in delineating the role played by the thixotropic/rheopectic behavior of the core fluid on unstable modes. To that end, with no loss of generality, we fix the dimensionless pressure gradient at $\Pi=-1$.

\subsection{Newtonian/Newtonian (N/N) Case}

For this case, we assume that the fluid flowing in the core and through the pore are both Newtonian and of the same viscosity and density values; that is: $\mathrm{K}_{\mathrm{v}}=1, \Lambda_{\mathrm{f}}=1$, unless otherwise is stated. Obviously, the stability data obtained for this particular case can serve as the baseline with which any non-Newtonian effects can be assessed for $\mathrm{M} / \mathrm{N}$ and $\mathrm{Q} / \mathrm{N}$ cases. Having said this, it should be conceded that the $\mathrm{N} / \mathrm{N}$ results have their own merit both because of their technological relevance and also because there is no such data available in the open literature. To that end, we start with presenting typical basic flow results for the N/N case. Figure 5 shows basic-flow flux profiles obtained for a typical rigid, porous layer $\left(\Gamma^{-1} \approx 0, \phi=0.7\right)$. As can be seen in Fig. 5 a, by an increase in the permeability parameter more fluid flows through the porous region, as expected. And, this gives rise to an increase in the flux through the main channel. The same is true as to the effect of the layer's thickness. That is, by an increase in thickness the flux increases in the layer and also in the core of the channel, as can be seen in Fig. 5b.
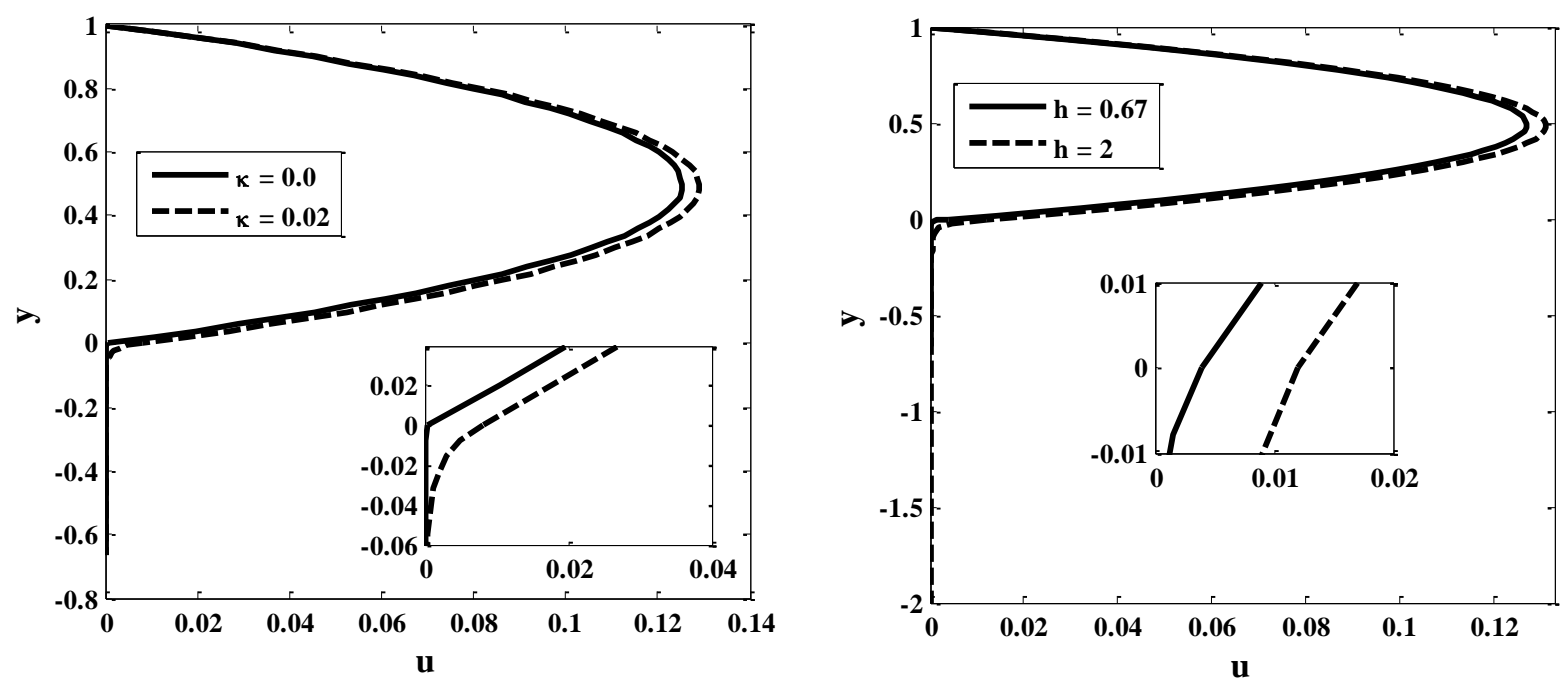

Figure 5: Typical basic-flow velocity profiles for the N/N case:

(a) effect of the permeability parameter, $\kappa$, obtained for $\mathrm{h}=0.67, \phi=0.7, \Gamma^{-1} \approx 0, \mathrm{~K}_{\mathrm{v}}=1$,

(b) effect of the layer thickness, h, obtained for $\kappa=0.01, \phi=0.7, \Gamma^{-1} \approx 0, \mathrm{~K}_{\mathrm{v}}=1$. 
Having found the basic flow/deformation for the N/N case, we can now proceed with investigating its vulnerability to instability when perturbed slightly. Figure 6 presents typical data showing the effect of the permeability parameter, $\kappa$, on the neutral instability curve for a rigid porous layer (more results can be found in Ref. 33). Figure 6 shows that the critical Reynolds number is affected by the layer's permeability in a non-trivial fashion. That is to say that, by an increase in the layer's permeability (starting from zero) the flow initially becomes more stable (cf. results for $\kappa=0$ and $\kappa=0.004$ ). But, by a further increase in the permeability its effect becomes destabilizing (see Fig. 6). An important aspect of the results presented in Fig. 6 is that, the neutral stability curves do not exhibit any kind of bi-modal characteristics. This is in contrast to the results reported by Chang et al [12] and Liu et al [13] who have predicted bi- and tri-modal behavior at sufficiently large Reynolds numbers. Tilton and Cortelezzi [16] has blamed the peculiar results reported in Refs. 12 and 13 on an improper use of two different length scales for rendering the governing equations dimensionless. Our length scale is the same as that used by Tilton and Cortelezzi [16], and this alone can explain why there is no multi-valued stability curves in our work. Another reason for the absence of bi-modal behavior in our neutral stability curves can be attributed to the fact that our analysis is based on the biphasic theory which treats boundary conditions and inertia terms quite differently to that used in Refs. 12 and 13. Still, none of these subtle issues concerns us that much in the present work. What concerns us most is the notion that the dominant mode and the critical Reynolds can be captured by our methodology - whether or not at sufficiently large Reynolds number a bi-modal behavior might emerge.

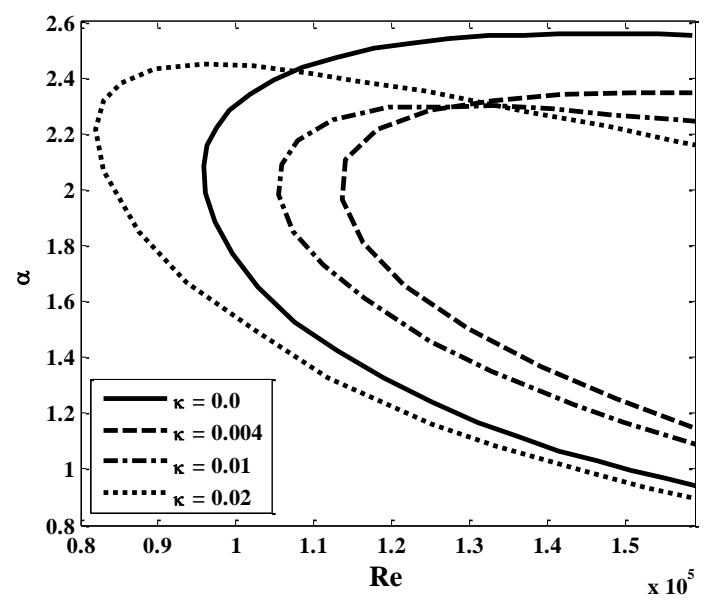

Figure 6: Effect of the permeability parameter, $\kappa$, on the neutral stability curve for the N/N case obtained at: $\Pi=-1, \mathrm{~h}=0.67, \phi=0.7, \Gamma^{-1} \approx 0$.

From the results presented in Fig. 6 it can be concluded that there exists a threshold permeability number (roughly 0.004 , for this set of parameters) beyond which permeability has a destabilizing effect on the main flow. It might be speculated that the threshold permeability depends on the porosity, thickness, and viscosity ratio. Figure 7a shows that this is not true as far as porosity is concerned. That is, the threshold permeability is practically independent of the layer's porosity; that is, it remains roughly at 0.005 even though the porosity itself is seen to have a stabilizing/destabilizing effect on the flow depending on its magnitude being smaller or larger than a critical value (roughly 0.015 for this set of parameters). Unlike porosity, Fig. 7b shows that an increase in the layer's thickness is always destabilizing. That is, for any given permeability parameter, the critical Reynolds number is decreased when the layer becomes thicker. 
This figure also shows that, for any given thickness the critical Reynolds number initially increases by an increase in the permeability although again we are witnessing a threshold value which decreases with an increase in the layer's thickness (say, from roughly 0.002 to roughly 0.005 ). Figure $7 \mathrm{c}$ shows that, like porosity and thickness, the cross-viscosity ratio $\left(\mathrm{K}_{\mathrm{v}}\right)$ also affects the threshold permeability. But, the effect is predicted to be stabilizing or destabilizing depending on the magnitude of the permeability number. Obviously, the effect of this parameter is much more complicated than the other two parameters, as can be seen in this figure.

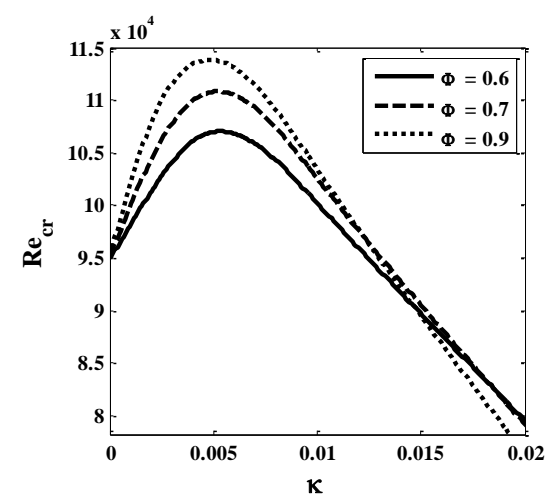

(a)

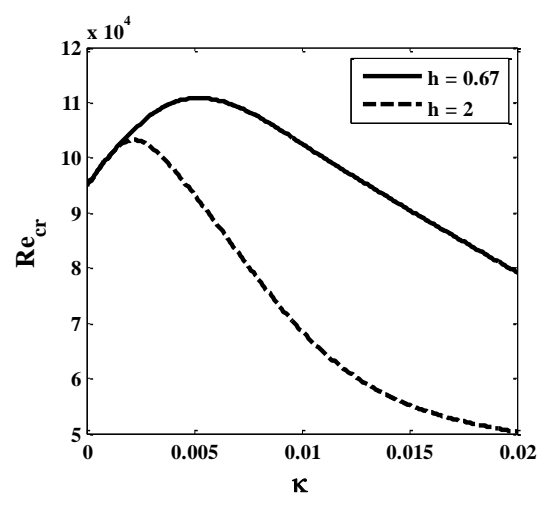

(b)

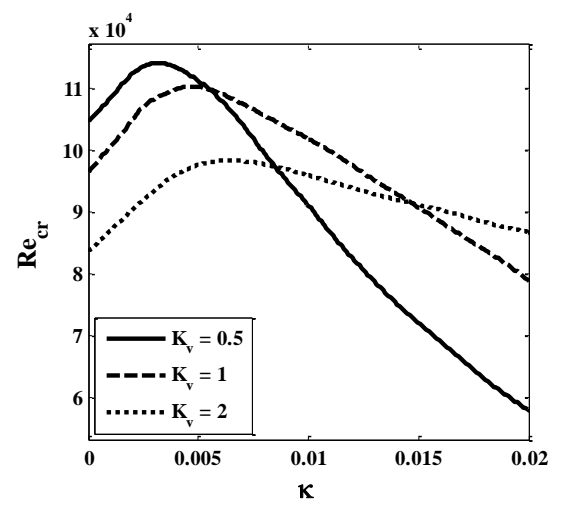

(c)

Figure 7: Dependence of the threshold permeability number on the properties of a rigid layer $\left(\Gamma^{-1} \approx 0\right)$ :

(a) effect of porosity, $\phi$, obtained for: $\mathrm{h}=0.67, \mathrm{~K}_{\mathrm{v}}=1, \Pi=-1$,

(b) effect of layer's thickness, h, obtained for: $\phi=0.7, \mathrm{~K}_{\mathrm{v}}=1, \Pi=-1$,

(c) effect of cross-viscosity, $\mathrm{K}_{\mathrm{v}}$, obtained for: $\mathrm{h}=0.67, \phi=0.7, \Pi=-1$.

Figure 8a shows how the growth rate of the critical mode corresponding to $\kappa=0.01$ varies with the Reynolds number. This figure also shows how the same mode grows with the Reynolds number when the permeability parameter is altered. As can be seen in this figure, near the critical Reynolds number (i.e., $1.03125 \times 10^{5}$ ) there is a sharp increase in the growth rate, as expected. Still, the largest growth corresponds to larger permeability numbers demonstrating that permeability has a destabilizing effect on the flow for this set of parameters. Interestingly, by a further increase in the Reynolds number, all four cases become eventually stable demonstrating that there is no bi-modal behavior in the neutral curve. Figure $8 \mathrm{~b}$ shows the spectrum of the eigenvalues and how they are affected by the permeability of the layer. Based on this figure, it can be concluded that wall permeability affects the phase speed ( $\mathrm{c}=-\omega / \mathrm{i \alpha})$ quite significantly. This figure shows that, all modes shown in Fig. 3 for the classic nonporous case are affected by the layer's porosity with the so-called damped modes being the ones which are more dramatically affected (see Fig. 8). This figure also shows the eigenvalue corresponding to the most dangerous mode (which is equal to: $\mathrm{c}=\mathrm{c}_{\mathrm{r}}+\mathrm{ic}_{\mathrm{i}}=0.06552+\mathrm{i}\left(-1.25 \times 10^{-12}\right.$ ), has been highlighted by a green circle in this figure. 

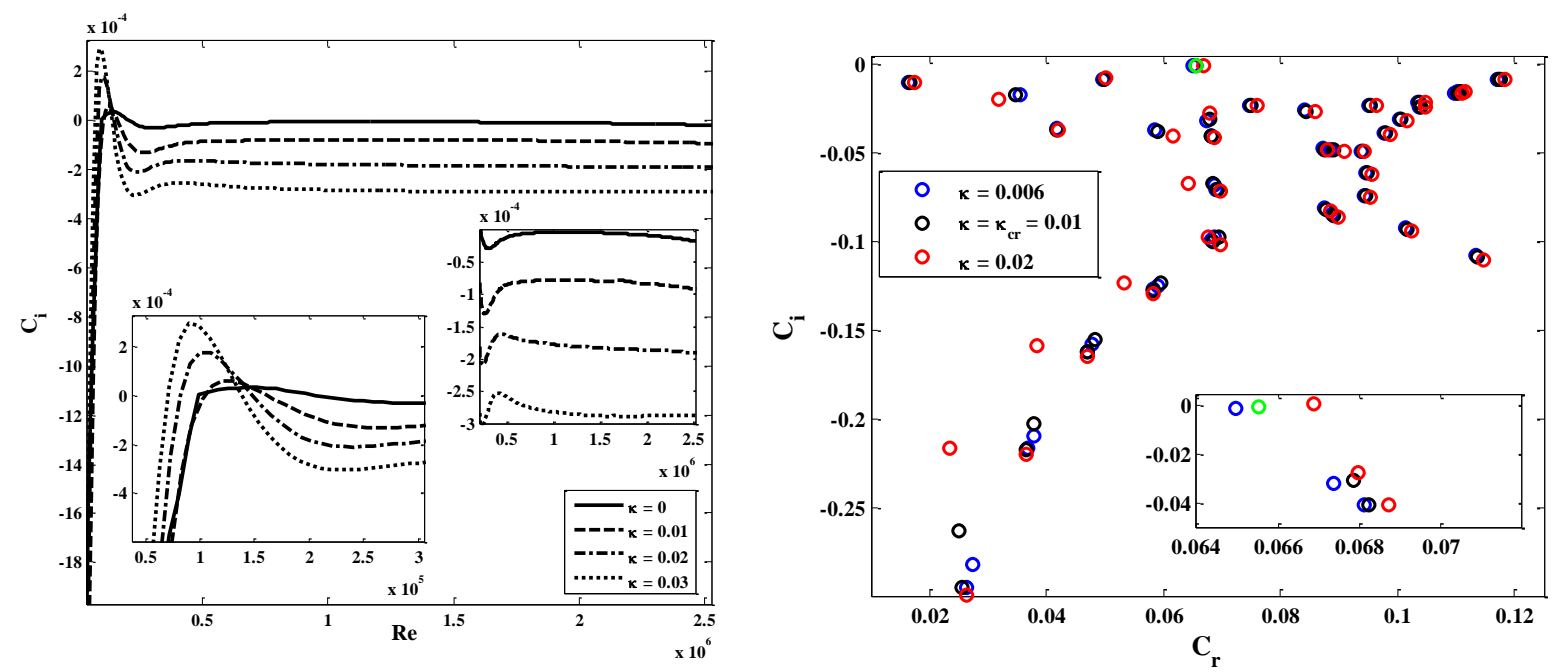

Figure 8: Variation of the phase speed (or growth rate) with the Reynolds number obtained for the critical wavenumber of $\alpha_{\text {cr }}=2.04$ (left plot); spectrum of the eigenvalues (right plot).

These results correspond to: $\mathrm{h}=0.67, \phi=0.7, \Gamma^{-1} \approx 0$.

Having investigated the effect of permeability on the instability picture for a porous but rigid layer we now proceed with investigating the effect of the layer's flexibility on the flow instability. Figure 9 shows the neutral stability curves obtained at a typical permeability of $\kappa=0.01$ for several flexibility numbers, $\Gamma^{-1}$. As can be seen in Fig. 9, by an increase in the poroelastic layer's flexibility, the flow becomes progressively more stable.

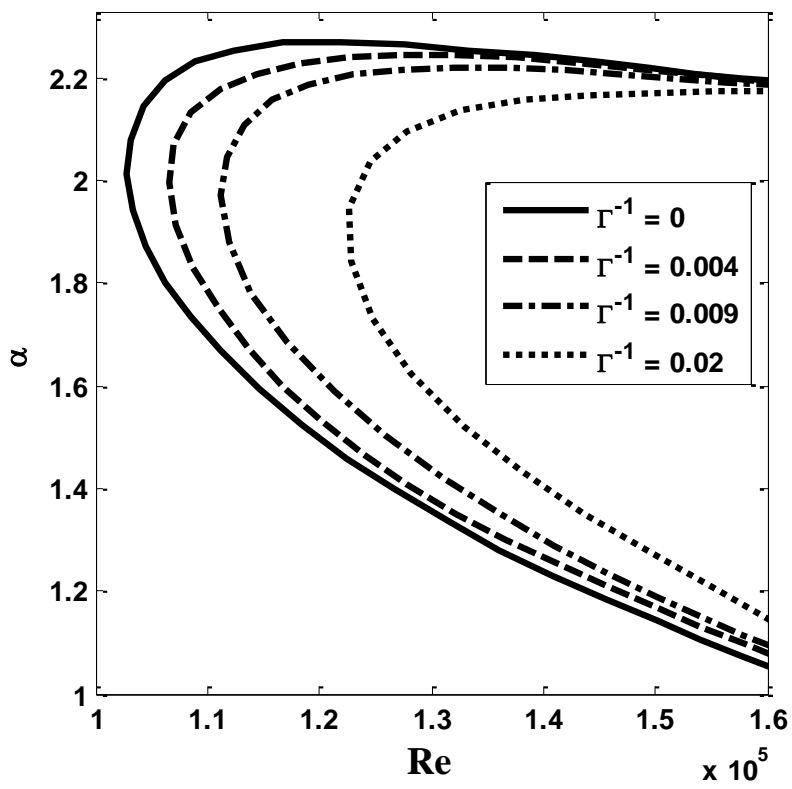

Figure 9: Effect of the flexibility parameter $\left(\Gamma^{-1}\right)$ on the neutral stability curve obtained at $\kappa=0.01, \mathrm{~h}=0.67, \phi=0.7, \mathrm{~K}_{\mathrm{v}}=1, \Lambda_{\mathrm{f}}=\Lambda_{\mathrm{s}}=1, \Pi=-1$. 
Figure 10 shows the effect of the layer's flexibility on the critical Reynolds number obtained for three different permeability numbers. This figure shows that, for any given permeability, the flow becomes monotonically more stable when the layer's flexibility is increased. Interestingly, however, this figure suggests that if the permeability is large enough, the critical Reynolds number may remain virtually intact when flexibility is increased (see results at $\kappa=0.02$ ). More importantly, this figure shows that the effect of permeability can be stabilizing or destabilizing depending on its magnitude and also the magnitude of the flexibility. For example, if we compare results obtained at $\kappa=0$ with those obtained at $\kappa=0.01$ we realize that for a narrow range of flexibility parameters (say, up to roughly 0.5 ) a permeable layer is more stable than an impermeable layer. Obviously, for sufficiently flexible layers, an increase in permeability destabilizes the flow.

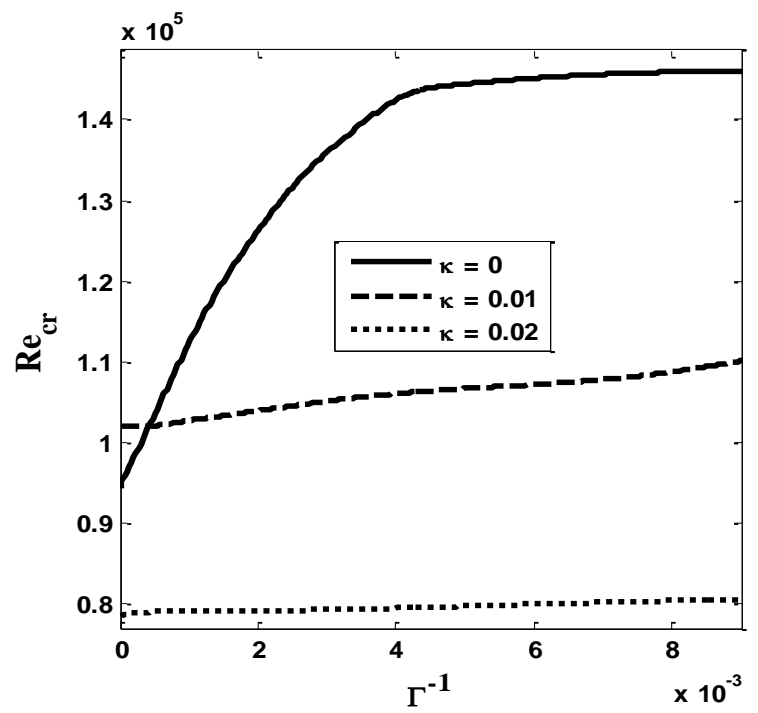

Figure 10: Variation of the critical Reynolds number with the flexibility number, $\Gamma^{-1}$, obtained at different permeability numbers $(\mathrm{h}=0.67, \varphi=0.7)$.

Figure 11a shows how the critical Reynolds number is affected when the solid's density ratio $\left(\Lambda_{\mathrm{s}}\right)$ is increased. Based on this figure, by an increase in the solid's density, the flow becomes progressively more stable at any given flexibility parameter. Figure $11 \mathrm{~b}$ shows that porosity has a similar effect on the instability picture. That is, for any given flexibility, an increase in the layer's porosity has a stabilizing effect on the main flow, at least, as long as porosity is in this range. (In Ref. 33, it has been shown that by approaching a porosity of one, it is possible that the effect of porosity becomes destabilizing.) 


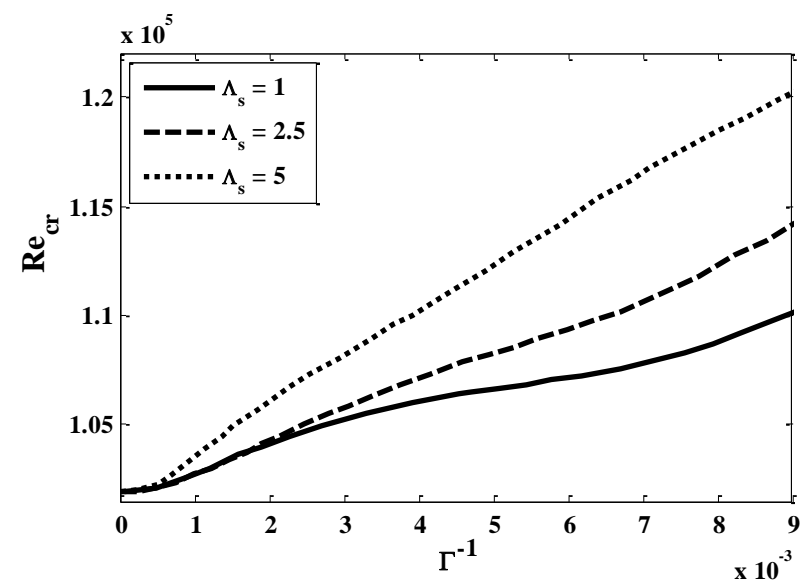

(a)

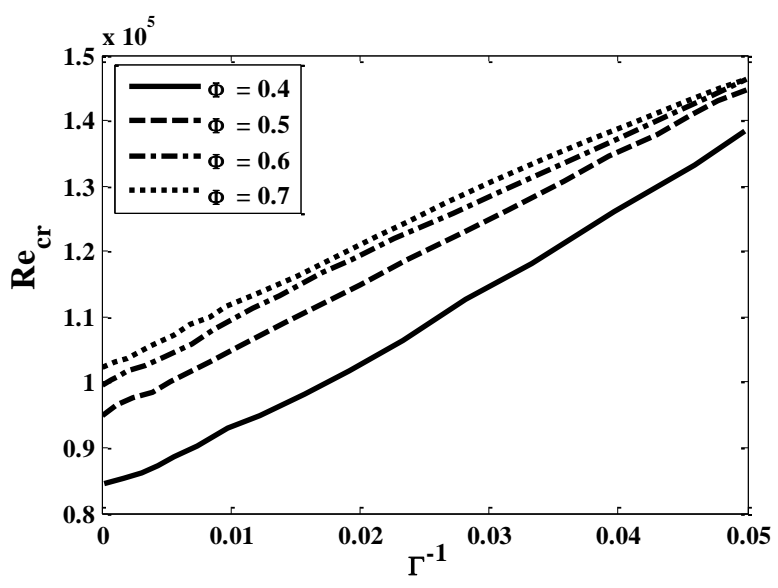

(b)

Figure 11: Variation of the critical Reynolds number with the flexibility number, $\Gamma^{-1}$, obtained for:

(a) three different solid density ratios $\left(h=0.67, \varphi=0.7, \Lambda_{\mathrm{f}}=1, \kappa=0.01\right)$.

(b) three different porosity $\left(\mathrm{h}=0.67, \kappa=0.01, \Lambda_{\mathrm{f}}=\Lambda_{\mathrm{s}}=1\right)$.

Figure 12 depicts how the phase speed (or, growth rate) varies with the Reynolds number when the flexibility number is varied. This figure shows that near the critical Reynolds number of $1.0312 \times 10^{5}$, there is a sharp increase in the growth rate, as expected. This figure also suggests that the largest growth rate corresponds to the rigid case, as previously mentioned. More importantly, by a further increase in the Reynolds number all four cases plotted in this figure become eventually stabilized demonstrating that there is no bi-modal behavior in the neutral stability curve, at least for this set of parameters. Figure 12 also shows the spectrum of eigenvalues and how they are influenced by the flexibility parameter. Again, the stabilizing effect of flexibility is evident in this figure.
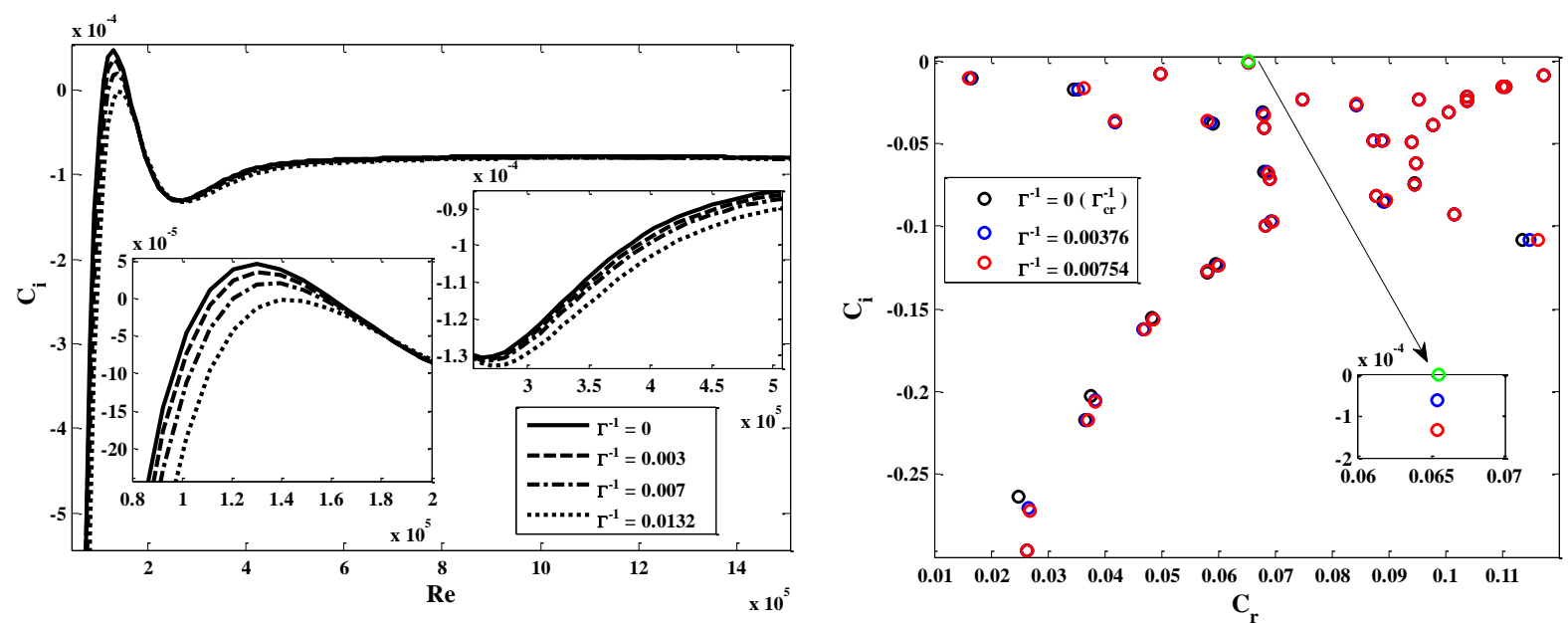

Figure 12: Variation of the growth rate with the Reynolds number for different flexibility numbers (left plot); spectrum of the eigenvalues obtained for $\operatorname{Re}_{c}=1.0312 \times 10^{5}$ (right plot).

Parameters are set at: $\Pi=-1, \mathrm{~h}=0.67, \varphi=0.7, \kappa=0.01, \mathrm{~K}_{\mathrm{v}}=1, \Lambda_{\mathrm{s}}=\Lambda_{\mathrm{f}}=1$.

To elucidate the mechanisms involved in stabilizing or destabilizing the flow, in Fig. 13 we have shown the velocity eigenvectors across the poroelastic layer obtained at the critical Reynolds number of 
$\mathrm{Re}_{\mathrm{cr}}=1.015 \times 10^{5}$ ). As can be seen in this figure, by a small increase in the layer's flexibility, there is a huge increase in the perturbed tangential velocity at the interface while, at the same time, the perturbed normal velocity is decreased. It can be argued that an increase in the perturbed tangential velocity has a stabilizing effect on the flow through increasing the Darcy drag. Similarly, a drop in perturbed normal velocity should also have a stabilizing effect as it lowers momentum transfer to the core fluid. Thus, it can be argued that both effects help to stabilize the flow. This is not the case when permeability is altered (see Fig. 14).
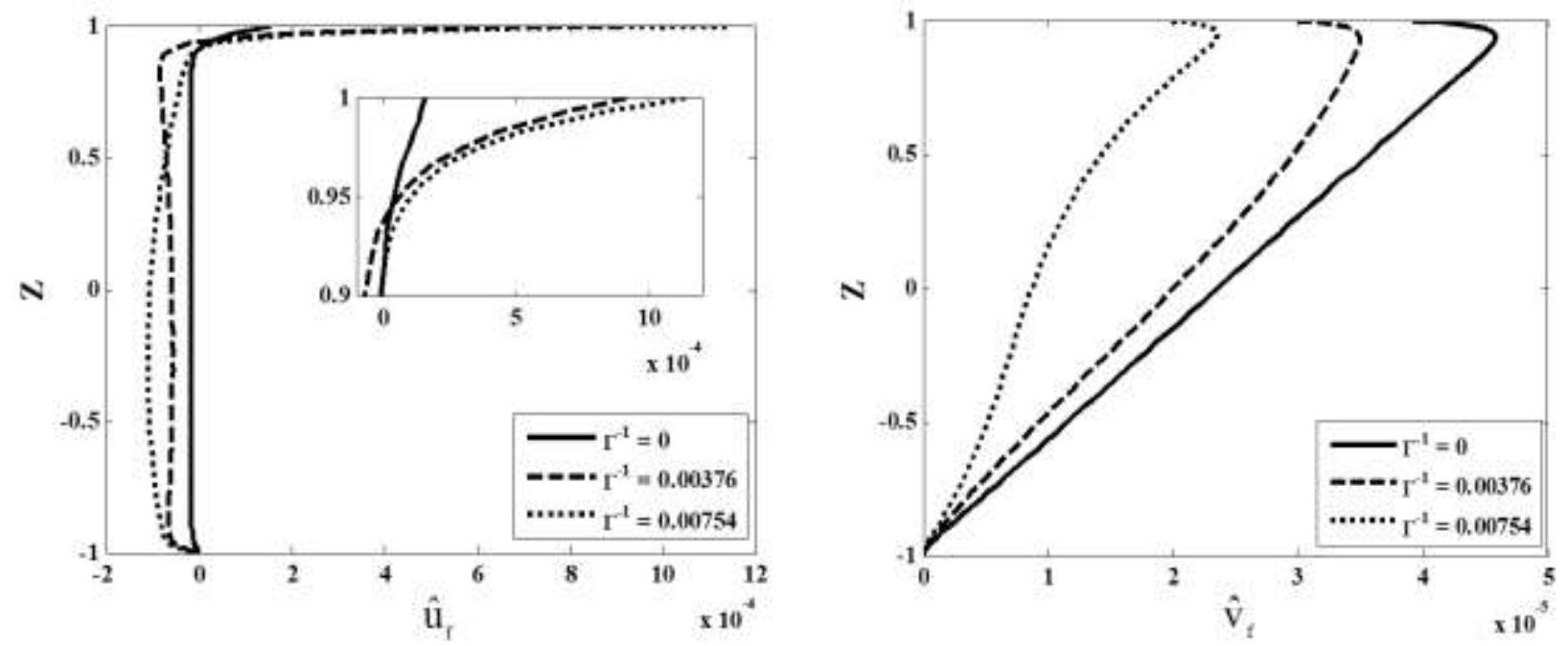

Figure 13: Effects of the flexibility parameter on the eigenfunction profiles across the poroelastic layer for the fluid flowing in the layer. In this figure $\mathrm{Z}=1$ refers to the location of the interface. These results have been obtained at the critical Reynolds number of $\mathrm{Re}_{\text {or }}=1.015 \times 10^{5}$ for the parameter setting of:

$$
\Pi=-1, \mathrm{~h}=0.67, \varphi=0.7, \kappa=0.01, \Lambda_{\mathrm{s}}=1 \text {, and } \mathrm{K}_{\mathrm{v}}=1 .
$$

Figure 14 shows the velocity eigenvectors across the poroelastic layer obtained at the critical Reynolds number of $\left.\operatorname{Re}_{\text {cr }}=9.4592 \times 10^{4}\right)$. As can be seen in this figure, by a small increase in the layer's permeability starting from zero (say, at $\kappa=0.004$ ) we are witnessing an initial increase in the perturbed tangential velocity. But, surprisingly, it starts to decrease when permeability is further increased to $\kappa=0.01$. Such peculiar behavior is not observed in the perturbed normal velocity. That is to say that, it increases at the interface when permeability is increased. So, it is evident that the non-monotonic results shown in Fig. 9 as to the effect of permeability on the neutral stability curve is closely controlled by $\hat{\mathrm{u}}_{\mathrm{f}}$ rather than $\hat{v}_{f}$. 

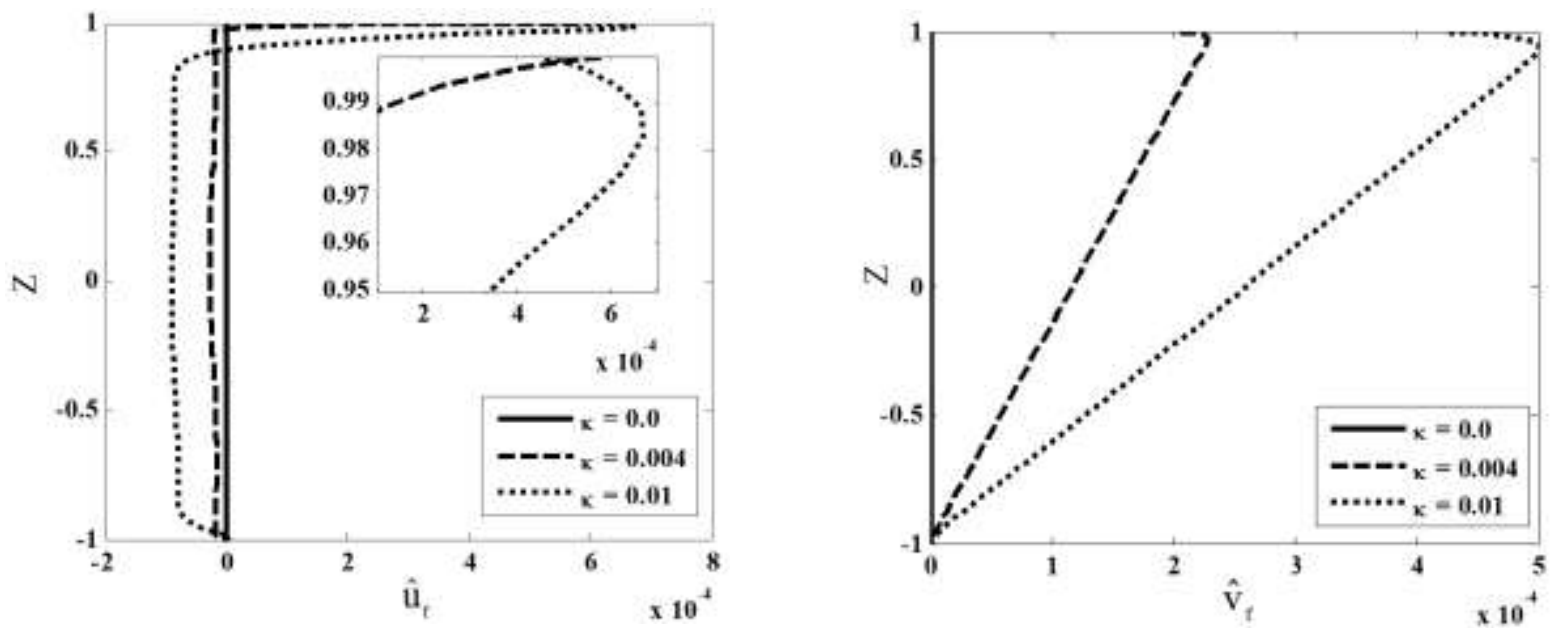

Figure 14: Effects of the permeability parameter on the eigenfunction profiles across the poroelastic layer for the fluid flowing in the layer. In this figure $\mathrm{Z}=1$ refers to the location of the interface. These results have been obtained at the critical Reynolds number of $\mathrm{Re}_{\mathrm{cr}}=9.4592 \times 10^{4}$ for the parameter setting of:

$$
\Gamma^{1} \approx 0, \Pi=-1, \mathrm{~h}=0.67, \varphi=0.7, \mathrm{~K}_{\mathrm{v}}=1=\Lambda_{\mathrm{s}} \operatorname{Re}_{\text {cr }}=9.4592 \times 10^{4} .
$$

Finally, we would like to stress that we have also investigated the effect of the Lame's constant $\chi$ in Eq. 4 (as represented by the dimensionless parameter $\theta$ in the governing equations) on the instability characteristics and reached to the conclusion that it has no measurable effect even on the basic flow; see Ref. 33. This finding corroborates Barry's finding [21] that this parameter (or, equivalently, the Poisson ratio) is of no consequence on flow through poroelastic layers as long as the deformation is infinitesimally small, as is the case in linear stability studies.

\subsection{Moore/Newtonian (M/N) Results}

In this section, we address the effect of the Moore model parameters on the critical Reynolds number for the channel shown in Fig. 1. To start with, in Fig. 15 we have shown the effect of viscosity-gap ratio $(\varepsilon)$ and thixotropy number (Tx) on the basic-flow flux profiles for a typical parameter setting of: $\varphi=0.7, \kappa=0.01, \mathrm{~K}_{\mathrm{v}}=1, \mathrm{~h}=0.67$, and $\Gamma^{-1}=0.009$. As can be seen in this figure, the flux profiles are strongly affected by these two parameters. An increase in the viscosity-gap ratio means that (for a given $\mu_{\infty}$ ) the zero-shear viscosity, $\mu_{0}$, is being increased. As such, it is of no surprise that the flux is lowered when this ratio is increased (see Fig. 15a). The effect of Tx is also not surprising because an increase in Tx means that the fluid becomes progressively more shear-thinning (see Appendix A). 

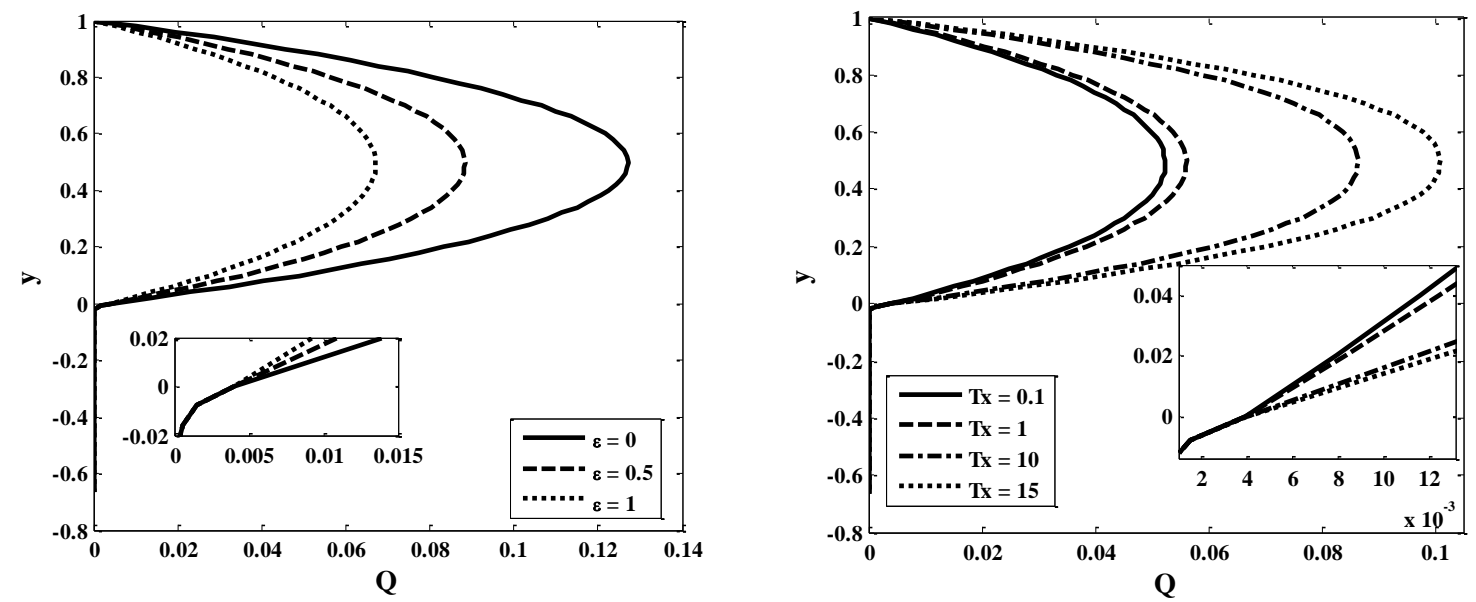

Figure 15: Typical basic-flow flux profiles for the $\mathrm{M} / \mathrm{N}$ case: effect of the viscosity-gap ratio obtained at $\mathrm{Tx}=0.5$ (left plot); effect of the thixotropy number obtained at $\varepsilon=1.5$ (right plot).

Knowing the basic solution, we now proceed with investigating their effect on the stability characteristics of the flow in the main channel. Figure 16 shows the effect of the thixotropy number on the critical Reynolds number for different solid density ratios and solid flexibility parameters. This figure shows that, like the N/N case, for any given Tx number the flow becomes progressively more stable when solid's density and/or its flexibility is increased. But, what is more interesting is the notion that for any given density ratio or flexibility parameter, an increase in the Tx number (starting from 0.1 ) causes a decrease in the critical Reynolds number, and this is true for thixotropy numbers up to $\mathrm{Tx}=10$. At $\mathrm{Tx}=15$, however, we are witnessing an increase in the critical Reynolds number. Since a Moore fluid practically behaves like a shear-thinning fluid at this extreme, one can conclude that the effect of shear-thinning is stabilizing (see, also, Appendix A).

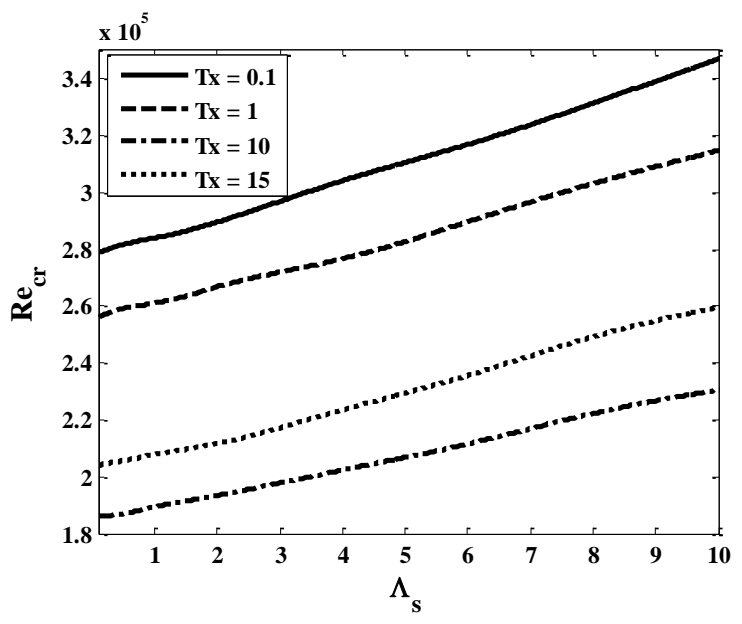

(a)

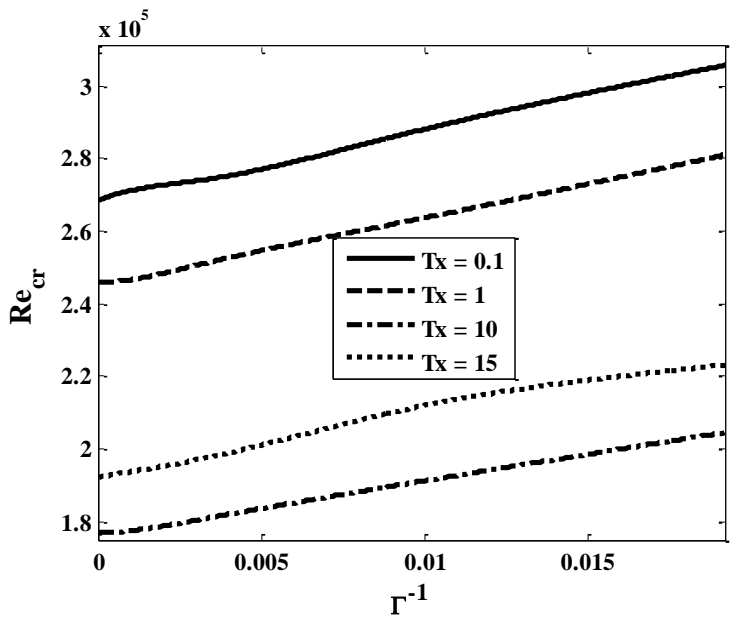

(b)

Figure 16: Effect of thixotropy number, Tx, on the critical Reynolds number obtained for:

(a) different solid density ratios at $\left(\Pi=-1, \mathrm{~h}=0.67, \phi=0.7, \mathrm{~K}_{\mathrm{v}}=1, \varepsilon=1.5, \kappa=0.01, \Gamma^{-1}=0.009\right)$.

(b) as a function of flexibility $\left(\Pi=-1, \mathrm{~h}=0.67, \phi=0.7, \mathrm{~K}_{\mathrm{v}}=1, \varepsilon=1.5, \kappa=0.01, \Lambda_{\mathrm{s}}=1\right)$. 
Figure 17 shows similar plots this time with the layer's porosity and thickness serving as the $\mathrm{x}$-axis. Figure 17a shows that, like the N/N case, an increase in the layer's thickness as a destabilizing effect on the flow for the $\mathrm{M} / \mathrm{N}$ case. In contrast, the effect of porosity is seen to be stabilizing or destabilizing depending on its size being smaller or larger than a threshold value (see Fig. 17b). The effect of Tx is seen to be destabilizing up to $\mathrm{Tx}=10$. At $\mathrm{Tx}=15$, however, we are again witnessing a stabilizing effect.

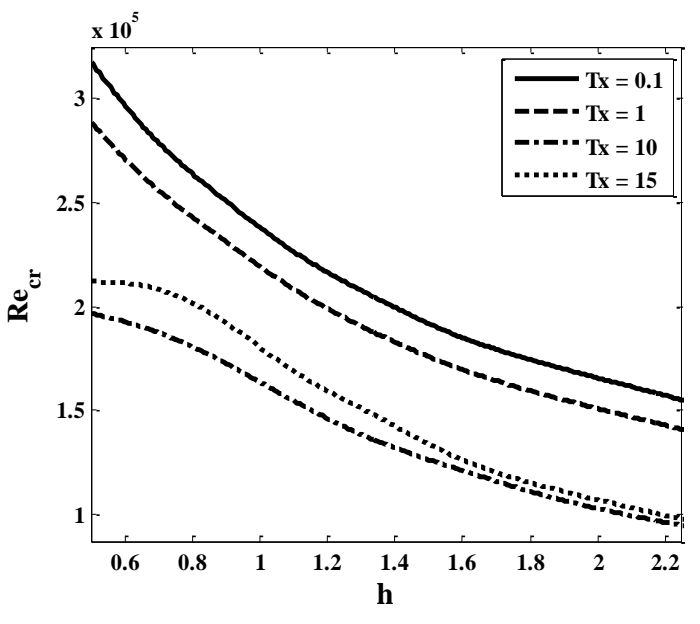

(a)

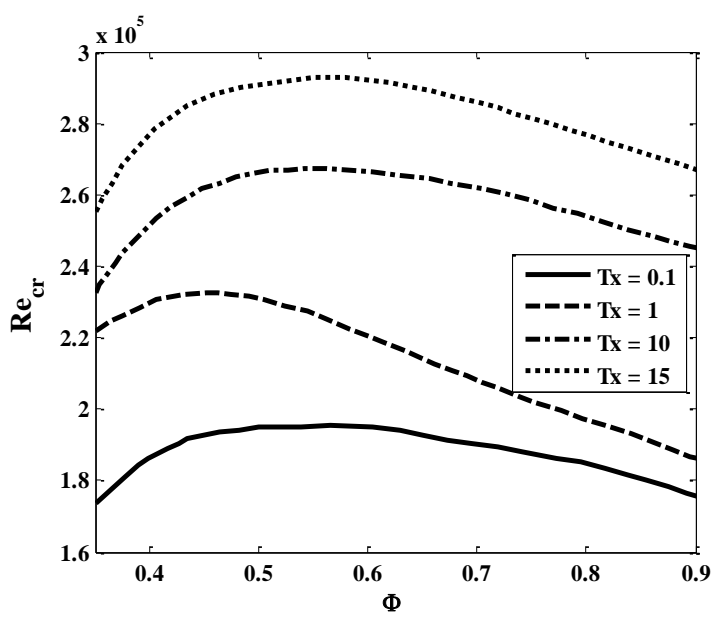

(b)

Figure 17: Effect of the thixotropy number, Tx, on the critical Reynolds number for the N/M case:

(a) as a function of porosity $\left(\Pi=-1, \varphi=0.7, \mathrm{~K}_{\mathrm{v}}=1, \kappa=0.01, \varepsilon=1.5, \Lambda_{\mathrm{s}}=1, \Gamma^{-1}=0.009\right.$ ).

(b) as a function of thickness $\left(\Pi=-1, \mathrm{~h}=0.67, \kappa=0.01, \varepsilon=1.5, \Lambda_{\mathrm{s}}=1, \Gamma^{-1}=0.009\right)$.

Figure 18 shows that the situation, as depicted above, becomes much more complicated when the layer's permeability or the cross-viscosity ratio is involved. As can be seen in Fig. 18a, at sufficiently small Tx numbers (say, at $\mathrm{Tx}=0.1$ ) an increase in the permeability parameter lowers the critical Reynolds number. But, when the Tx number is sufficiently large (say, $\mathrm{Tx}=10$ ) it initially stabilizes the flow followed by a destabilizing effect if permeability is larger than a threshold value. As to the effect of the cross-viscosity ratio, Fig. 18b shows that for Tx up to 10, it has a stabilizing effect (cf. results for Tx $=0.1$ with $\mathrm{Tx}=10$ ). For any given $\mathrm{Tx}$ in this range, the effect of $\mathrm{K}_{\mathrm{v}}$ is initially stabilizing but becomes eventually destabilizing if it is large enough. For $\mathrm{Tx}=15$, however, the effect of $\mathrm{K}_{\mathrm{v}}$ is monotonically destabilizing. Also, for certain values of $\mathrm{K}_{\mathrm{v}}$ it becomes more stable than $\mathrm{Tx}=10$. 


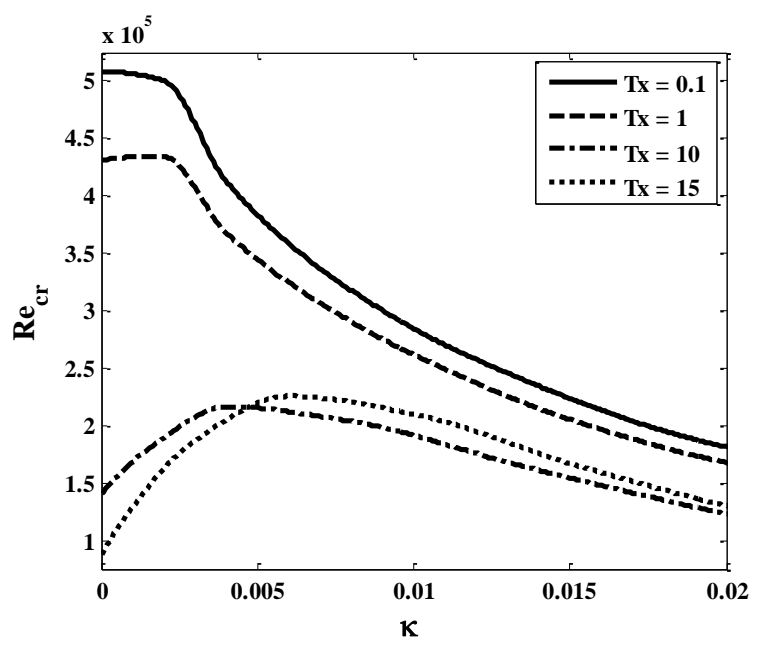

(a)

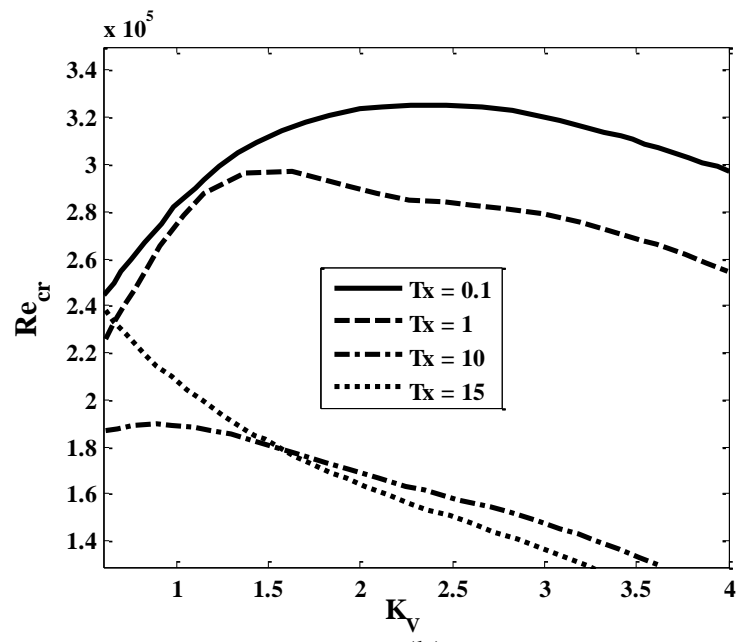

(b)

Figure 18: Effect of the thixotropy number, Tx, on the critical Reynolds number for the $\mathrm{M} / \mathrm{N}$ case as a function of:

(a) permeability $\left(\Pi=-1, \mathrm{~h}=0.67, \varphi=0.7, \mathrm{~K}_{\mathrm{v}}=1, \varepsilon=1.5, \Gamma^{-1}=0.009\right)$.

(b) cross-viscosity $\left(\Pi=-1, \mathrm{~h}=0.67, \varphi=0.7, \kappa=0.01, \varepsilon=1.5, \Gamma^{-1}=0.009\right)$.

In Fig. 19, we have presented the eigenvalue spectrum for the typical case of $\varphi=0.7, \kappa=0.01, \varepsilon=1.5, \mathrm{Tx}=0.5, \Gamma^{-1} \approx 0, \Pi=-1, \mathrm{~h}=0.667$. For this particular case, the critical Reynolds number is found to be equal to: $\mathrm{Re}_{\mathrm{cr}}=2.569 \times 10^{5}$ for which the eigenvalue is: $\mathrm{C}_{\mathrm{r}}=0.02913, \mathrm{C}_{\mathrm{i}} \approx 0$. This figure also shows results obtained at two other Reynolds numbers: one below the critical $\operatorname{Re}$ and the other above it. This figure shows that for $\operatorname{Re}=2.370 \times 10^{5}$ we have $\mathrm{C}_{\mathrm{r}}=0.02922, \mathrm{C}_{\mathrm{i}}=-0.007744$ (a stable mode) and for $\mathrm{Re}=2.796 \times 10^{5}$ it is equal to: $\mathrm{C}_{\mathrm{r}}=0.02905, \mathrm{C}_{\mathrm{i}}=+0.00036$ (an unstable mode). Obviously, by an increase in Re, a mode which is stable or neutrally stable is becoming unstable.

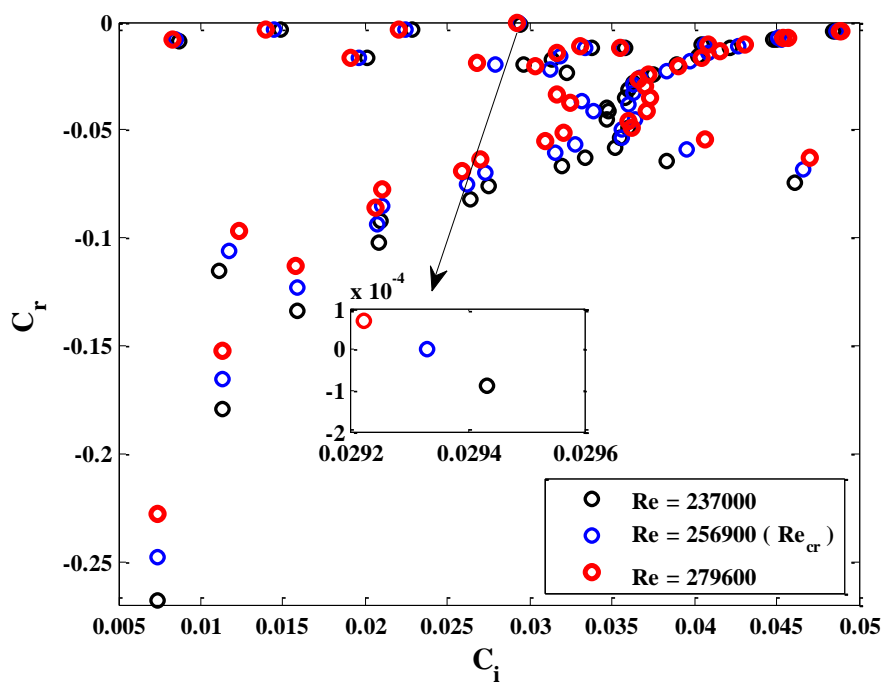

Figure 19: Spectrum of the eigenvalues for the N/M case obtained at $\varphi=0.7, \kappa=0.01, \varepsilon=1.5, \mathrm{Tx}=0.5, \Gamma^{-1} \approx 0, \Pi=-1, \mathrm{~h}=0.667:$ 
The above results were mostly concerned with the effect of the thixotropy number, Tx, on the instability behavior of our FSI problem. This was because of the importance of the characteristic time of the Moore model (b/a) which appears in the Tx number. For curiosity, in Fig. 20 we have addressed the effect of the dimensionless rebuild parameter, $\Theta$, on the neutral stability curve for two different thixotropy numbers, $\mathrm{Tx}=0.05$ and 0.5 . For the case of $\mathrm{Tx}=0.05$, which corresponds to the more thixotropic case, this figure shows that the critical Reynolds number is barely affected by $\Theta$. At $\mathrm{Tx}=0.5$, however, the stabilizing effect of $\Theta$ becomes a little bit more pronounced. Still, looking at the scale of the vertical axis, one can conclude that the stabilizing effect of $\Theta$ is negligible (see Fig. 20).

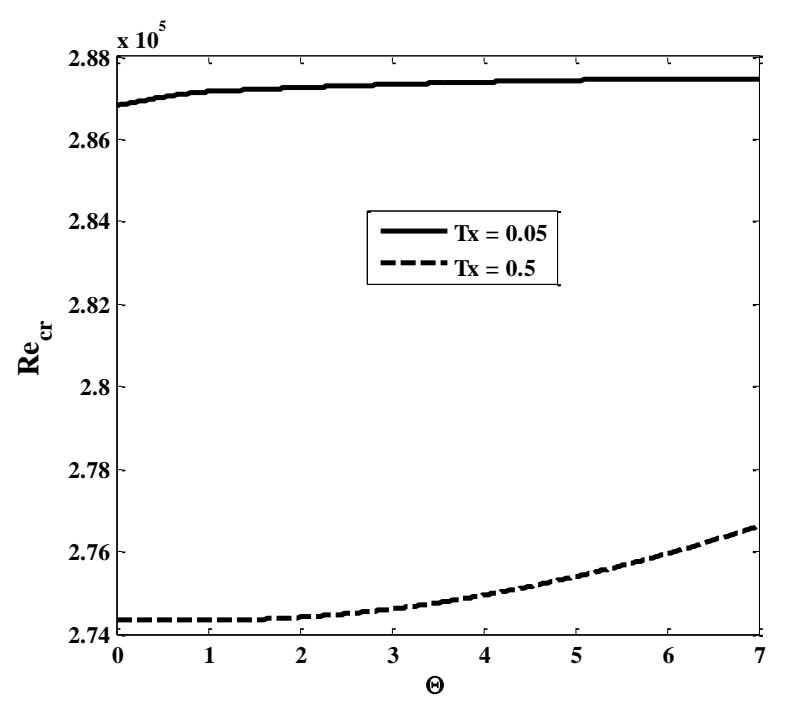

Figure 20: Effect of the rebuild parameter, $\Theta$, on the neutral stability curve obtained for

$$
\varepsilon=1.5, \Pi=-1, \mathrm{~h}=0.67, \kappa=0.01, \Gamma^{-1}=0.009 \text {. }
$$

Having investigated the effect of the thixotropy number, we now investigate the effect of the viscositygap ratio in the Moore model $(\varepsilon)$ on the critical Reynolds number. As mentioned earlier, for a given $\mu_{\infty}$ an increase in $\varepsilon$ means an increase in the zero-shear viscosity, $\mu_{0}$. As can be seen in Fig. 21, regardless of the flexibility, permeability, porosity, and the solid's density in the poroelastic layer, the viscosity-gap ratio always has a stabilizing effect on the main flow. Since by an increase in $\varepsilon$, the apparent viscosity of the Moore fluid is increased, one can conclude that core fluid's viscosity has a stabilizing effect on the flow. 


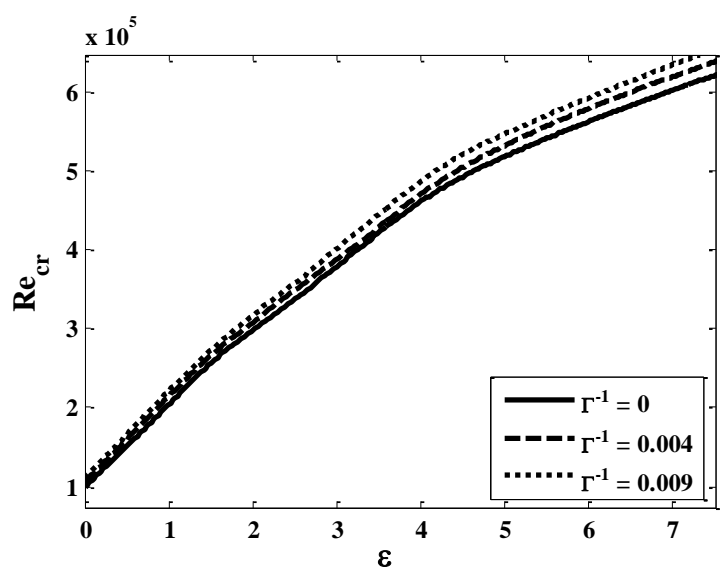

(a)

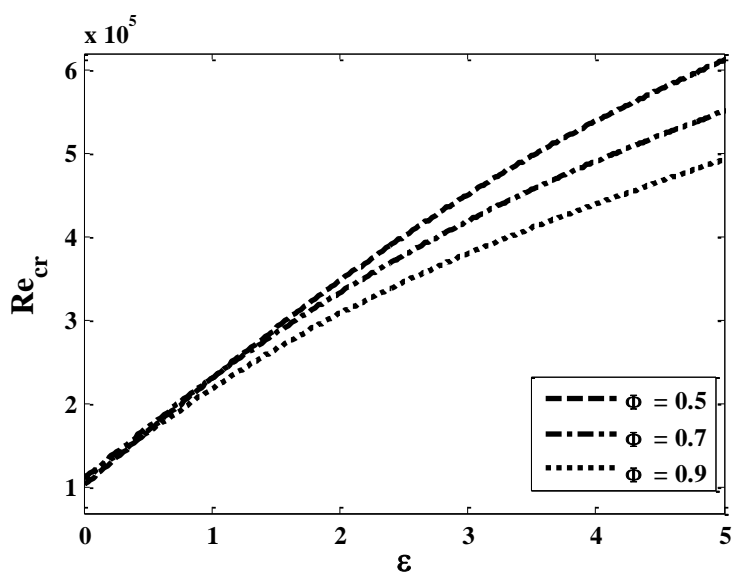

(c)

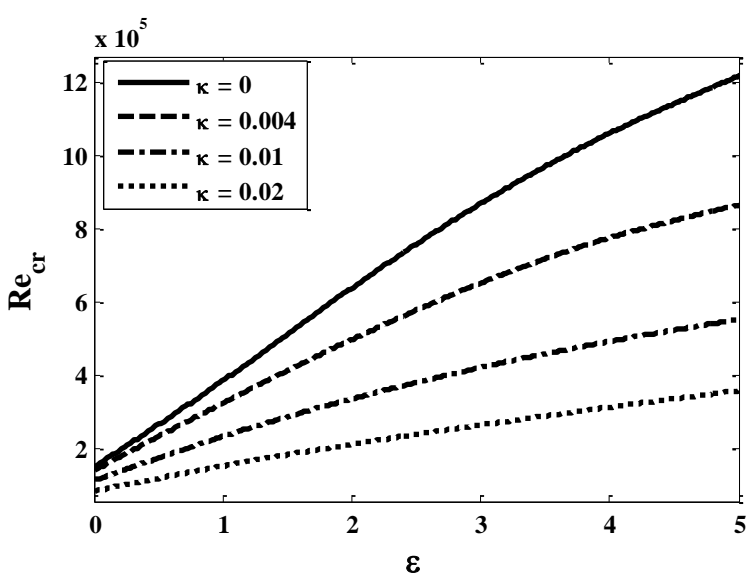

(b)

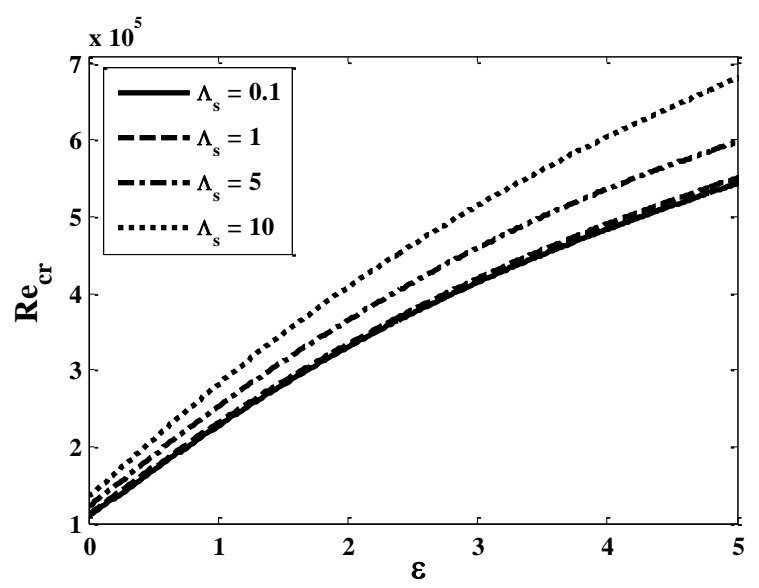

(d)

Figure 21: Effect of the viscosity-gap ratio, $\varepsilon$, on the critical Reynolds number for the N/M case:

(a) for different flexibility parameter $\left(\Pi=-1, \mathrm{~h}=0.67, \phi=0.7, \mathrm{Tx}=0.1, \mathrm{~K}_{\mathrm{v}}=1\right)$,

(b) for different permeability $\left(\Pi=-1, \mathrm{~h}=0.67, \mathrm{~K}_{\mathrm{v}}=1, \phi=0.7, \mathrm{Tx}=0.1, \Lambda_{\mathrm{s}}=1, \Gamma^{-1}=0.009\right.$ ),

(c) for different porosity $\left(\Pi=-1, \mathrm{~h}=0.67, \mathrm{~K}_{\mathrm{v}}=1, \kappa=0.01, \mathrm{Tx}=0.1, \Lambda_{\mathrm{s}}=1, \Gamma^{-1}=0.009\right)$,

(d) for different solid density $\left(\Pi=-1, \mathrm{~h}=0.67, \mathrm{~K}_{\mathrm{v}}=1, \varphi=0.7, \kappa=0.01, \mathrm{Tx}=0.1, \Gamma^{-1}=0.009\right.$ ).

The above figure clearly shows that $\varepsilon$ in the Moore model plays a definitive role on the instability picture. In fact, we have reached to the conclusion that if $\varepsilon, \varphi$, and $\mathrm{h}$ are large enough, there is no peak in the Re- $\kappa$ curve, as predicted to exist for the N/N case (see Figs. 7a,b). As can be seen in Fig. 22a, for $\varepsilon=4$ and $\varphi=$ 0.9 the effect of permeability is monotonically destabilizing. Similarly, Fig. 22b shows that for $\varepsilon=4$ and $\mathrm{h}=2$ permeability always destabilizes the flow. 


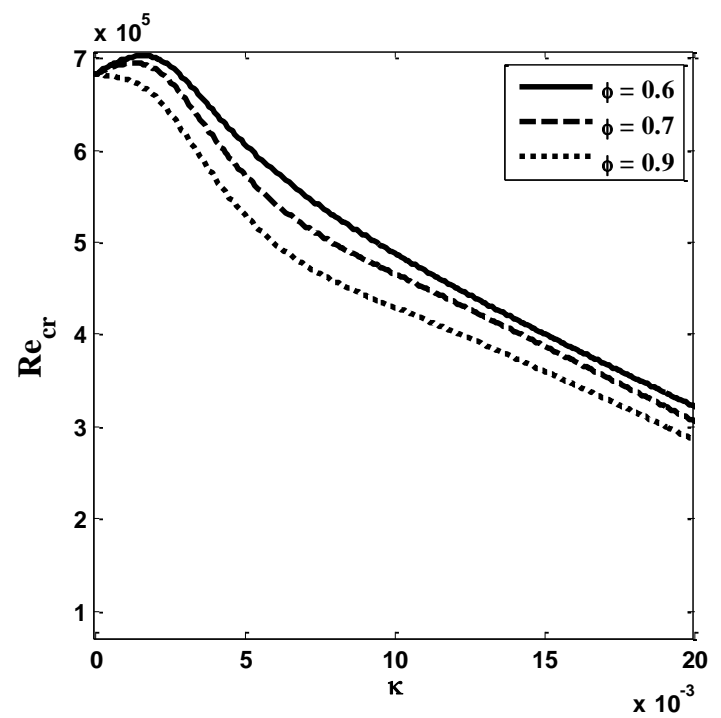

(a)

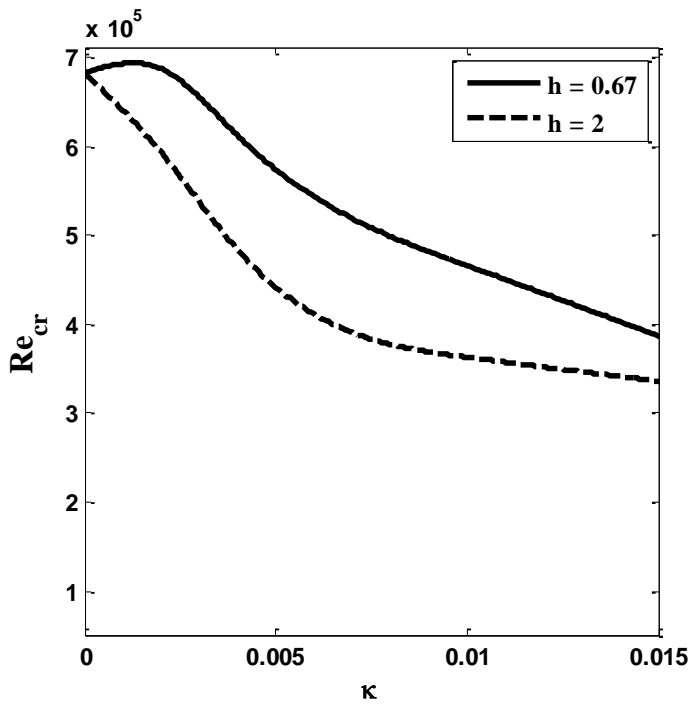

(b)

Figure 22: Effect of the permeability parameter on the critical Reynolds number for three different porosity ratios for the $\mathrm{M} / \mathrm{N}$ case obtained at: $\Pi=-1, \varepsilon=4, \mathrm{Tx}=0.5, \mathrm{~h}=0.67, \mathrm{~K}_{\mathrm{v}}=1, \Gamma^{-1} \approx 0$ :

(a) effect of porosity, (b) effect of thickness.

\section{Concluding Remarks}

In the present work, we have tried to investigate the effect of a poroelastic lining (fixed to the lower wall of a two-dimensional channel formed by two rigid, infinitely long parallel plates of infinite width) on the stability picture of the main flow. A common negative pressure gradient was assumed to drive both fluids. The fluid flowing through the main channel was assumed to obey the Quemada model whereas the one flowing through the poroelastic layer was Newtonian. The solid matrix was assumed to obey the linear elastic model. We relied on the biphasic mixture theory to come up with the required boundary conditions (both kinematic and dynamic) at the interface. The same theory was also used to develop the equations of motion in the poroelastic region. A linear temporal stability analysis was invoked to find the critical Reynolds number and to what extent they are affected by the parameters of the poroelastic lining (say, its porosity, permeability, flexibility, density, and thickness) and the rheological properties of the working fluids. Our main concern was to discern the competition which exists between flexibility and permeability in stabilizing/destabilizing the flow. And, we have kept all inertia terms in the analysis so as to make it more comprehensive. Having said this, it should be conceded the common practice in our community is to start from the creeping-flow analysis and then to extend it to the inertial analysis in subsequent works. (We have decided to go for the hardest scenario simply because in our recent publication dealing with compliant layers [20] we have already shown that the creeping-flow scenario may give rise to unrealistic behavior in the neutral stability curve.) We have reached to the following main conclusions:

- Permeability has a stabilizing or destabilizing effect on the main flow depending on its magnitude being smaller or larger than a threshold. The threshold permeability was found to be rather insensitive to porosity, but it is decreased by an increase in the layer's thickness. 
- Flexibility of the poroelastic layer has a stabilizing effect on the flow which becomes weaker by an increase in the layer's permeability.

- Porosity can have a stabilizing or destabilizing effect depending on the layer's permeability.

- An increase in the solid's density has a stabilizing effect on the flow.

- An increase in the layer's thickness has a destabilizing effect on the flow.

- Thixotropy has a stabilizing effect on the flow with rheopexy having an opposite effect.

- Shear-thinning has a stabilizing effect on the flow.

- Yield stress has a stabilizing effect on the flow.

- The viscosity-gap ratio always has a stabilizing effect on the core flow.

- The effect of the cross-viscosity ratio can be stabilizing or destabilizing depending on the permeability number.

- The main cause of instability is the (streamwise and transverse) velocity perturbations at the interface. Our eigenfunction velocity profiles have revealed that between the two, it is the tangential velocity perturbations and its huge variation by the permeability and flexibility of the layer which closely controls the stability picture in poroelastic layers.

The most important finding of the present work is the prediction that, as far as linear stability of poroelastic layers is concerned, the case of boundary layer flows is markedly different from the case of channel flows. That is, while for the former case poroelastic layers always have a stabilizing effect on the flow, for the latter case its effect can be stabilizing or destabilizing depending on the layer's physical parameters and the rheology of the two fluids involved in the problem. We would also like to stress that, although we have tried to make the present work as comprehensive as possible, we admit that the work suffers from certain shortcomings. To us, the main shortcoming of the present work is the notion that, due to numerical difficulty (caused by a change of length scale near the interface due to the formation of a boundary layer) we could not obtain stability results at very large permeability numbers. Fortunately, however, the range of permeability parameters for which numerical results could be obtained is typical of physiological and drag-reduction applications. Another shortcoming of the work is the notion that we have relied on the linear elasticity theory for quantifying the deformation of the porous skeleton. Although this model is good for representing physiological systems and drag-reduction coatings, we are aware that there are cases of practical interest where hyperelastic models should be used instead. Having said this, we believe that the results obtained in this work are still very useful in that they can be used as a baseline with which future works dealing with hyperelastic models can be compared with.

From the above shortcomings, one can infer that the present work can be extended in several ways. Obviously, the case of large-permeability might interest many researchers, and so it can be the subject of an independent work. Similarly, the case of hyperelastic models can also be the subject of a thorough and independent study. As a matter of fact, one might also be tempted to try the case of creeping flow and see if an omission of inertia terms gives rise to any kind of peculiar behavior in the neutral stability curve. Although we have been able to comment on the stabilizing effect of shear-thinning and yield stress using the Moore model, we admit that this is not a specialized model for addressing such effects. We believe 
more specialized rheological models such as power-law or Bingham must be used in subsequent works to thoroughly investigate such important effects. Similarly, the case in which both plates are lined with the same poroelastic layer or even with layers having different properties and thicknesses might interest some researchers for different purposes. In addition, the fluid flowing through the poroelastic layer can be allowed to obey a non-Newtonian model if the need arises. Other geometries such as plane Couette flow (where pressure gradient is of no consequence) might also interest some investigators; see Refs. 52 and 53. The role played by anisotropy and/or inhomogeneity of the poroelastic layer might also interest some researcher. As a matter of fact, we are currently trying to extend the present work to the case of anisotropic/inhomogeneous permeability.

\section{Acknowledgement:}

The authors wish to express their sincerest thanks to Iran National Science Foundation (INSF) for supporting this work under contract number: 95849145. Special thanks are also due to the respectful reviewers for their encouraging and constructive comments. Finally, it would be a remiss not to mention the fruitful discussion we had with Prof. S.M. Taghavi (Laval University, Department of Chemical Engineering) in the course of this work for which we are grateful.

\section{Nomenclature:}

$\begin{array}{ll}\text { a } & \text { Rebuild parameter } \\ \text { b } & \text { Destruction parameter } \\ \text { C } & \text { Phase speed } \\ \text { D } & \text { Rate-of-deformation tensor } \\ \text { E } & \text { Young's modulus of elasticity } \\ \mathrm{G} & \text { Pressure gradient } \\ \mathrm{H} & \text { Height of the unperturbed channel } \\ \mathbf{I} & \text { Identity tensor } \\ \mathrm{k} & \text { Destruction-to-rebuild ratio, b/a } \\ \mathrm{K} & \text { Permeability } \\ \mathrm{K}_{\mathrm{v}} & \text { Cross-viscosity ratio } \\ \mathrm{M}, \mathrm{N} & \text { Chebyshev terms } \\ \mathrm{m}, \mathrm{n} & \text { Parameters in the Quemada model } \\ \mathbf{n} & \text { Unit vector normal to the interface } \\ \mathrm{p} & \text { Fluid pressure in the main channel } \\ \mathrm{p}_{\mathrm{avg}} & \text { Pressure in the poroelastic layer } \\ \mathrm{Q} & \text { Fluid flux in the poroelastic layer } \\ \mathrm{R} & \text { Height of the poroelastic layer } \\ \mathrm{Re} & \text { Reynolds number } \\ \mathrm{S} & \text { Structural parameter } \\ \mathrm{T} & \text { Flow characteristic time } \\ \mathrm{Tx} & \text { Thixotropy number } \\ \mathbf{U} & \text { Velocity vector in the main channel } \\ \mathrm{u}_{\mathrm{f}} & \text { Fluid velocity in the x-direction in the poroelastic layer } \\ & \end{array}$




\begin{tabular}{|c|c|}
\hline $\mathrm{u}$ & Fluid velocity in the $\mathrm{x}$-direction in the main channel \\
\hline$v_{f}$ & Fluid velocity in the $y$-direction in the poroelastic layer \\
\hline $\mathrm{v}$ & Fluid velocity in the y-direction in the main channel \\
\hline $\mathbf{V}$ & Velocity vector in the poroelastic layer \\
\hline $\mathrm{X}_{\mathrm{x}}$ & Solid displacement in the $\mathrm{x}$-direction \\
\hline $\mathrm{X}_{\mathrm{y}}$ & Solid displacement in the y-direction \\
\hline Y & Coordinate transformation in the poroelastic layer \\
\hline $\mathrm{Z}$ & Coordinate transformation in the main channel \\
\hline$\alpha$ & Dimensionless wavenumber \\
\hline$\beta$ & Power law index of Quemada model \\
\hline$\chi$ & Lame's constant \\
\hline$\theta$ & Dimensionless Lame's constant \\
\hline$\xi$ & Horizontal component of the perturbed interface at $y=0$ \\
\hline$\delta$ & Vertical component of the perturbed interface at $y=0$ \\
\hline$\varepsilon$ & Viscosity-gap ratio \\
\hline$\varphi_{\mathrm{f}}$ & Fluid's volume fraction in the porolelastic layer \\
\hline$\varphi_{\mathrm{s}}$ & Solid's volume fraction in the porolelastic layer \\
\hline$\phi$ & Porosity \\
\hline$\eta$ & Apparent viscosity of the core fluid \\
\hline$\lambda$ & Relaxation time \\
\hline$\kappa$ & Dimensionless permeability \\
\hline$\rho_{\mathrm{f}}$ & Fluid's density in the poroelastic layer \\
\hline$\rho_{\mathrm{s}}$ & Solid's density in the poroelastic layer \\
\hline$\rho$ & Fluid's density in the main channel \\
\hline$\tau$ & Stress tensor \\
\hline$\sigma$ & Total stress tensor \\
\hline$\omega$ & Growth rate \\
\hline$\zeta$ & Dimensionless two-phase reaction factor \\
\hline$\dot{\gamma}$ & Second invariant of the rate-of-deformation tensor \\
\hline$\mu_{0}$ & Zero-shear viscosity \\
\hline$\mu_{\infty}$ & Infinite-shear viscosity \\
\hline$\mu_{\mathrm{f}}$ & Fluid's viscosity in the poroelastic layer \\
\hline$\Lambda_{\mathrm{f}}$ & Fluid density ratio in the poroelastic layer \\
\hline$\Lambda_{\mathrm{s}}$ & Solid density ratio in the poroelastic layer \\
\hline$\Gamma$ & Dimensionless shear modulus \\
\hline$\Theta$ & Dimensionless thixotropy time \\
\hline
\end{tabular}

Subscripts:

$\mathrm{f} \quad$ Fluid in the poroelastic layer

s Solid in the poroelastic layer 


$\begin{array}{ll}\mathrm{cr} & \text { Critical conditions } \\ \mathrm{i} & \text { Initial configuration } \\ \mathrm{e} & \text { Equilibrium configuration }\end{array}$

\section{References:}

[1] C.Y. Choi, P.M. Waller, Momentum transport mechanism for water flow over porous media, J. Environ. Eng., 123 (1997) 792-799.

[2] D.B. Das, V. Nassehi, R.J. Wakeman, A finite volume model for the hydrodynamics of combined free and porous flow in sub-surface regions, Adv. Environ. Res., 7 (2002) 35-58.

[3] M. Discacciati, E. Miglio, A. Quarteron, Mathematical and numerical models for coupling surface and ground water flows, Appl. Numer. Math., 43 (2002) 57-74.

[4] M. Le Bars, M.G. Worster, Interfacial conditions between a pure fluid and a porous medium: implications for binary alloy solidification, J. Fluid Mech., 550 (2006) 149-173.

[5] E. Miglio, A. Quarteroni, F. Saleri, Coupling of free surface and groundwater flows, Comput. Fluids, 32 (2003) 73-83.

[6] J.A. Ochoa-Tapia, S. Whitaker, Momentum transfer at the boundary between a porous medium and a homogeneous fluid. I. Theoretical development, Int. J. Heat Mass Transf., 38 (1995) 26352646.

[7] D.D. Joseph, L.N. Tao, Lubrication of a porous bearing-Stokes' solution, J. of Applied Mechanics, Series E, 88(3) (1966) 753-760.

[8] C.S. Alphonsus, and R. N. Rodseth, The endothelial glycocalyx: a review of the vascular barrier, Anaesthesia, 69 (2014) 777-784.

[9] A.J.S. Fox, A. Bedi, and S.A. Rodeo, The basic science of articular cartilage: structure, composition, and function, Sports Health, 1(6) (2009) 461-468.

[10] G.S. Beavers, E.M. Sparrow, and R.A. Magnuson, Experiments on coupled parallel flows in a channel and a bounding porous medium, Journal of Basic Engineering, Series D, 92 (1970) 843848.

[11] E.M. Sparrow, G.S. Beavers, T.S. Chen, and J.R. Lloyd, Breakdown of the laminar flow regime in permeable-walled ducts, J. Appl. Mech., 40 (1973) 337-342.

[12] M.H. Chang, F. Chen, and B. Straughan, Instability of Poiseuille flow in a fluid overlying a porous layer, J. Fluid Mech., 564 (2006) 287-303.

[13] R. Liu, Q. S. Liu, and S. C. Zhao, Instability of plane Poiseuille flow in a fluid-porous system, Phys. Fluids 20, (2008) 104105.

[14] L.M. de Socio, L. Marino, and G. Seminara, Stability and admittance of a channel flow over a permeable interface, Physics of Fluids, 17 (2005) 094103-7.

[15] N. Silin, J. Converti, D. Dalponte, and A. Clausse, Flow instabilities between two parallel planes semi-obstructed by an easily penetrable porous medium, J. Fluid Mech., 689 (2011) 417-433. 
[16] N. Tilton, and L. Cortelezzi, Linear stability analysis of pressure-driven flows in channels with porous walls, J. Fluid Mech., 604 (2008) 411-445.

[17] P. Deepu, P. Anand, and S. Basu, Stability of Poiseuille flow in a fluid overlying an anisotropic and inhomogeneous porous layer, Physical Review E, 92 (2015) 023009-10.

[18] M. Pierucci, P.G. Morales, Effect of finite thickness flexible boundary upon the stability of a Poiseuille flow, Trans. ASME: J. Appl. Mech., 57 (1990) 1056-1060.

[19] V. Gkanis, S. Kumar, Stability of pressure driven creeping flows in channels lined with a nonlinear elastic solid, J. Fluid Mech., 524 (2005) 357-375.

[20] M. Pourjafar, K. Sadeghy, Linear stability of shear-thinning fluids in deformable channels: effect of inertial terms, J. Non-Newt. Fluid Mech., 230 (2016) 80-91.

[21] A. Shrivastava, E.L. Cussler, and S. Kumar, Mass transfer enhancement due to a soft elastic boundary, Chem. Eng. Sci., 63 (2008) 4302-4305.

[22] S.I. Barry, K.H. Parker, G.K. Aldis, Fluid flow over a thin deformable porous layer, Journal of Applied Mathematics and Physics (ZAMP), 42 (1991) 633-648.

[23] J.S. Hou, M.H. Holmes, W.M. Lai, and V.C. Mow, Boundary conditions at the cartilage-synovial fluid interface for joint lubrication and theoretical verifications, J. Biomech. Eng., 111 (1989) 7887.

[24] F. Pluvinage, A. Kourta, and A. Bottaro, Instabilities in the boundary layer over a permeable, compliant wall, Physics of Fluids, 26 (2014) 084103-16.

[25] B. Albers, Linear stability analysis of a 1D flow in a poroelastic material under disturbances with adsorption, Proceedings of the 11th Conference on Waves and Stability in Continuous Media (WASCOM 2001), Porto Ercole (Grosseto), Italy, 3-9 June 2001.

[26] A. Samanta, S. Bagheri, and L. Brandt, Linear stability of a liquid flow through a poroelastic channel, 15th European Turbulence Conference, 25-28 August, 2015, Delft, The Netherlands.

[27] PEL-SKIN: A novel kind of surface coatings in aeronautics, European Commission, Contract No. 334954, Funded under: FP7-TRANSPORT, 2013-2015.

[28] V. Shankar and S. Kumar, Instability of viscoelastic plane Couette flow past a deformable wall, J. Non-Newtonian Fluid Mech., 116 (2004) 371-393.

[29] S.A. Roberts and S. Kumar, Stability of creeping Couette flow of a power-law fluid past a deformable solid, J. Non-Newtonian Fluid Mech., 139 (2006) 93-102.

[30] M. Pourjafar, H. Hamedi, K. Sadeghy, Stability of power-law fluids in creeping plane Poiseuille flow: The effect of wall compliance, Journal of Non-Newtonian Fluid Mech., 216 (2015) 22-30.

[31] H.H. Wei, S.L. Waters, S.Q. Liu, and J.B. Grotberg, Flow in a wavy-walled channel lined with a poroelastic layer, J. Fluid Mech., 492 (2003) 23-45.

[32] K.A. Athanasiou, R.M. Natoli, Introduction to Continuum Biomechanics, Morgan \& Claypool publishers, 2008. 
[33] M. Pourjafar, Linear Stability of Non-Newtonian Fluids in Channels Equipped with Poroelastic Layers, PhD thesis, in progress.

[34] J. Schurz, V. Ribitsch, Rheology of synovial fluid, Biorheology, 24(4) (1987) 385-99.

[35] E.W. Merrill, Rheology of blood, Physiol. Rev., 49 (1969) 863- 888.

[36] L.T. Wardhaugh, D.V. Boger, S.P. Tonner, Rheology of waxy oils, Society of Petroleum Engineers, SPE, SPE-17625-MS, International Meeting on Petroleum Engineering, Tianjin, China, 1988.

[37] C. Baravian, D. Quemada and A. Parker, Modelling thixotropy using a novel structral kinetics approach: Basis and application to a solution of IOTA Carrageenan, Journal of Texture Studies, vol. 27, no. 4, pp. 371-390, 1996.

[38] D. Quemada, Rheological modelling of complex fluids. I. The concept of effective volume fraction revisited, Eur. Phys. J. AP, 1 (1998) 119-127.

[39] P. Neofytou, Comparison of blood rheological models for physiological flow simulation, Biorheology, 41(6) (2004) 693-714.

[40] A. Wachs, G. Vinay, I. Frigaard, A 1.5D numerical model for the start-up of weakly compressible flow of a viscoplastic and thixotropic fluid in pipelines, J. Non-Newton. Fluid Mech., 159 (2009) 81-94.

[41] F. Moore, The rheology of ceramic slips and bodies, Trans. Brit. Ceram. Soc., 58 (1959) 470-494.

[42] A. Mujumdar, A.N. Beris, A.B. Metzner, Transient phenomena in thixotropic systems, J. NonNewt. Fluid Mech., 102 (2002) 157-178.

[43] F. Moore, The rheology of ceramic slips and bodies, Trans. Br. Ceram. Soc. 58, (1959) 470.

[44] J.J. Derksen, Drag on random assemblies of spheres in shear-thinning and thixotropic liquids, Physics of Fluids, 21 (2009) 083302-9.

[45] P.G. Drazin, W.H. Reid, Hydrodynamic Stability. Cambridge: Cambridge University Press; 1981

[46] C. Canuto, M.Y. Hussaini, A, Quarteroni, T.A. Zang, Spectral Methods in Fluid Dynamics, New York: Springer; 1988.

[47] S. Orszag, Accurate solution of the Orr-Sommerfeld stability equation, J. Fluid Mech., 50(4) (1971) 659-703

[48] P. Schmid, and D. Henningson. Stability and Ttransition in Shear Flows. Springer, 2001.

[49] J.J. Derksen, Prashant, Simulations of complex flow of thixotropic liquids, J. Non-Newtonian Fluid Mech., 160 (2009) 65-75.

[50] C.R. Beverly, R.I. Tanner, Numerical analysis of three-dimensional Bingham plastic flow, J. Non-Newt. Fluid Mech., 42 (1992) 85-115.

[51] I. Frigaard, On the stability of shear flows of suspensions, XVth International Congress on Rheology, August 3-8, 2008, Monterey, California, USA. 
[52] V. Gkanis and S. Kumar, Instability of creeping Couette flow past a neo-Hookean solid, Phys. Fluids, 15 (2003) 2864-2871.

[53] V. Shankar and S. Kumar, Instability of viscoelastic plane Couette flow past a deformable wall, J. Non-Newtonian Fluid Mech., 116 (2004) 371-393.

\section{Appendix A: The Shear-Thinning Aspect of the Moore Model}

An important aspect of the Moore model is that it can simultaneously represent shear-thinning fluids in addition to representing thixotropic fluids. To see this in better perspective, we can calculate the equilibrium viscosity in steady shear for this model (see Eqs. 10a,b); the result is:

$\mu_{\mathrm{eq}}=\mu_{\infty}\left(1+\varepsilon\left(\frac{\mathrm{a}}{\mathrm{a}+\mathrm{b} \dot{\gamma}}\right)\right)=\mu_{\infty}\left(1+\frac{\varepsilon}{1+(\mathrm{b} / \mathrm{a}) \dot{\gamma}}\right)$

From the above equation it can be seen that, for any given $\varepsilon \neq 0$ a Moore fluid behaves practically like a shear-thinning fluid with the degree of shear-thinning being controlled by the coefficient b/a. Figure A1 shows the effect of $\mathrm{k}=\mathrm{b} / \mathrm{a}$ on the equilibrium viscosity obtained at $\varepsilon=10$. As can be seen in this figure, by an increase in $\mathrm{k}$ the degree of shear-thinning becomes more pronounced.

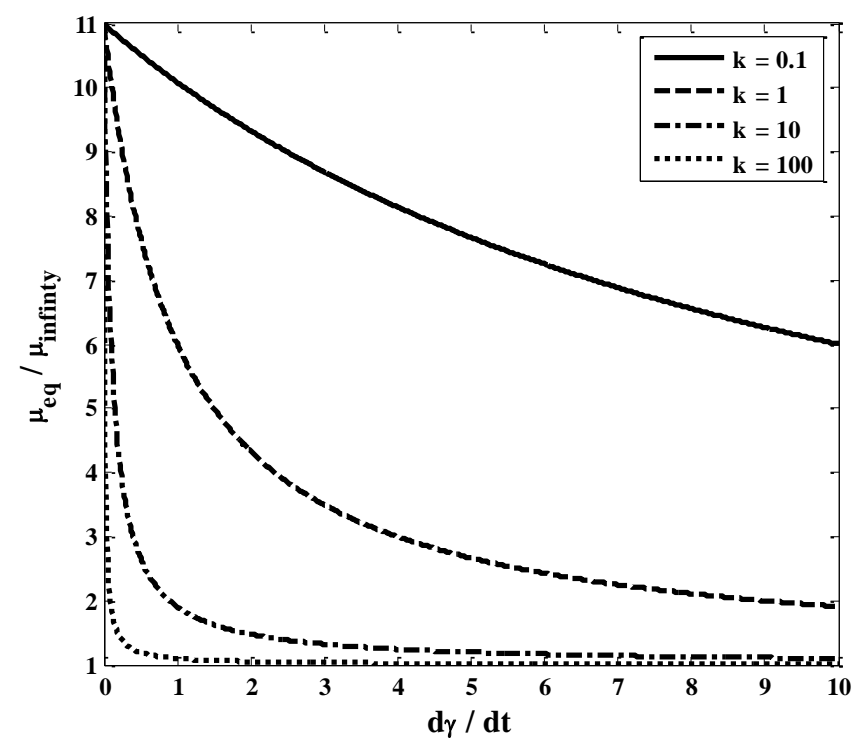

Figure A1: Effect of the b/a ratio (k) on the shear-thinning behavior of Moore fluid in simple shear flow $(\varepsilon=10)$.

Based on the results presented in Fig. A1, it can be said that a Moore fluid for which the ratio $k=b / a$ is large is more likely to exhibit shear-thinning effects. This is tantamount to saying that fluids for which the thixotropy number, Tx, is very large are exhibiting shear-thinning effects at the expense of thixotropic effects. Therefore, by obtaining results at this extreme we are actually addressing the effect of shear-thinning on any given flow. To investigate the effect of shear-thinning on the stability picture, it suffices to gradually increase the thixotropy number and see what happens to the critical Reynolds number. Figure A2 shows results obtained this way. First of all, it shows that flexibility and 
permeability have a stabilizing effect on the core flow for this range of Tx numbers. More importantly, Fig. A2 (left plot) suggests that, for any given flexibility parameter $\Gamma^{-1}$, the critical Reynolds number is decreased when Tx is increased until a critical Tx of roughly 10 is reached. The flow then becomes progressively more stable by a further increase in the Tx number up to Tx $=20$. From these results it can be concluded that thixotropy has a stabilizing effect on the flow (cf. results at $\mathrm{Tx}=0.1$ with $\mathrm{Tx}=0.2$ ). The fact that, beyond a $\mathrm{Tx}$ of roughly 10 , a further increase in $\mathrm{Tx}$ has a stabilizing effect on the flow suggests that the effect of shear-thinning is also stabilizing, as previously noted. An increase in $\Gamma^{-1}$ does not alter the overall picture although it stabilizes the flow for any given Tx. Figure A2 (right plot) suggests that similar conclusion can be made as to the effect of permeability parameter, $\kappa$.

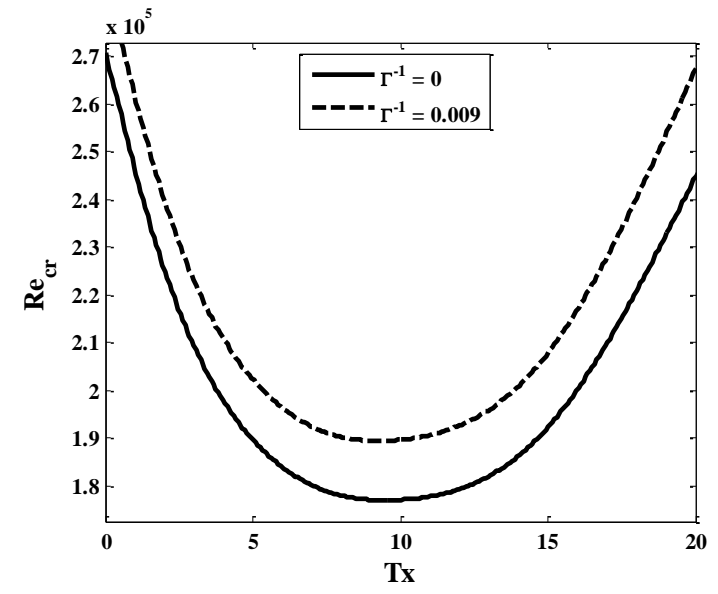

(a)

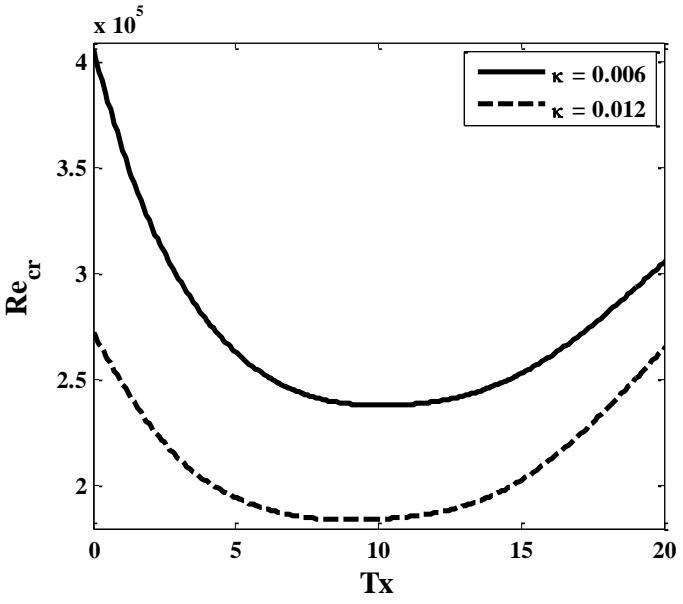

(b)

Figure A2: Effect of shear-thinning on the critical Reynolds number obtained for

$$
\varepsilon=1.5, \varphi=0.7, \mathrm{~h}=0.67, \mathrm{G}=-1: \text { (a) } \kappa=0.01 \text {, (b) } \Gamma^{-1}=0.018 \text {. }
$$

\section{Appendix B: The Viscoplastic Aspect of the Moore Model}

Dereksen and Prashant [49] have shown that Moore model can closely approximate the bi-viscous Bingham model frequently used for representing viscoplastic fluids. To elaborate on this issue, we start with the bi-viscous model which reads as [50]:

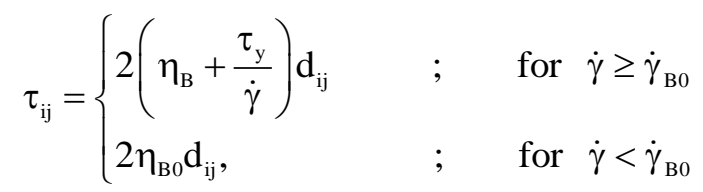

where $\eta_{B 0}$ is the (large) viscosity of the un-yielded region, and $\eta_{B}$ is the (small) viscosity of the yielded region. To ensure that the stress is continuous at $\dot{\gamma}_{\mathrm{B} 0}$ we should have [50]:

$$
\dot{\gamma}_{\mathrm{Bo}}=\frac{\tau_{\mathrm{y}}}{\eta_{\mathrm{B} 0}-\eta_{\mathrm{B}}}
$$


which means that $\dot{\gamma}_{\mathrm{B} 0}$ is fixed as soon as the values of $\eta_{\mathrm{B} 0}, \eta_{\mathrm{B}}$, and $\tau_{\mathrm{y}}$ are fixed. In practice, by choosing $\eta_{\text {в }} / \eta_{\text {в }}$ sufficiently large we are practically replacing the un-yielded zones with a highly-viscous fluid in this fluid model. Beverly and Tanner [50] have shown that for this analogy to be true, $\dot{\gamma}_{\text {в0 }}$ should be less than or equal to $10^{-3}$. Figure B1 shows schematically how, in simple shear, the flow-curve of a bi-viscous model compares with the flow curve of the classic Bingham model.

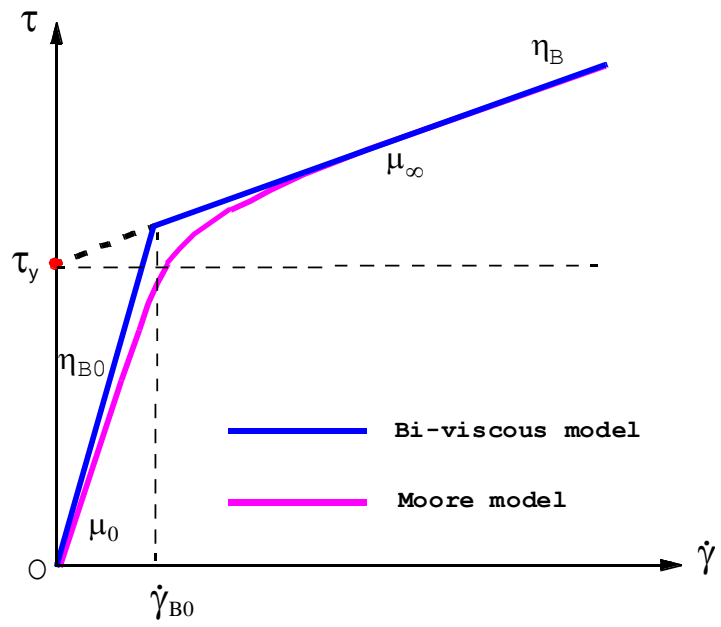

Figure B1: An analogy between the Moore model and the bi-viscous model in simple shear.

As can be seen in Fig. B1, the two models have much in common. As a matter of fact, by setting $\varepsilon=\left(\eta_{\mathrm{B} 0} / \eta_{\mathrm{B}}\right)-1, \mu_{\infty}=\eta_{\mathrm{B}}$, and $\mu_{0}=\eta_{\mathrm{B} 0}$, for the analogy to be complete it only remains to relate the ratio b/a to the yield stress, $\tau_{\mathrm{y}}$ and this can be done by simply setting: $\dot{\gamma}_{\mathrm{Bo}}=\dot{\gamma}_{\mathrm{c}}=\mathrm{a} / \mathrm{b}$ which renders: $\tau_{\mathrm{y}}=\left(\eta_{\mathrm{B}} \varepsilon\right) /(\mathrm{b} / \mathrm{a})$; and this completes the analogy. In order to investigate the effect of $\tau_{\mathrm{y}}$ on the instability picture, in practice, it suffices to increase $\varepsilon$ for a given b/a (say, 1000) and a given $\mu_{\infty}$ (say, 1). (We prefer not to alter b/a as it directly affects the thixotropic/shear-thinning behavior of the material.) This means that, for the yield stress to be of order one, $\varepsilon$ should be at least of order 1000. Figure B2 shows the effect of the Bingham number, Bn, on the basic flow flux profiles where the Bn number is defined as:

$$
\mathrm{Bn}=\frac{\tau_{\mathrm{Y}}}{\mathrm{HG}}=\frac{\varepsilon}{\mathrm{Tx}}
$$

This figure clearly shows that by an increase in Bn, plug-like zones appear in the middle of the core. Figure B3 shows the effect of Bn on the neutral stability curves. From this figure one can conclude that the effect of yield stress is stabilizing for this set of parameters. Having said this, we would like to stress that studying the effect of yield stress on the stability picture warrants an independent work using the biviscous or Bingham model instead of the Moore model. The preliminary results obtained using the Moore model only serve to show that the stability picture is very sensitive to a fluid's yield stress. 


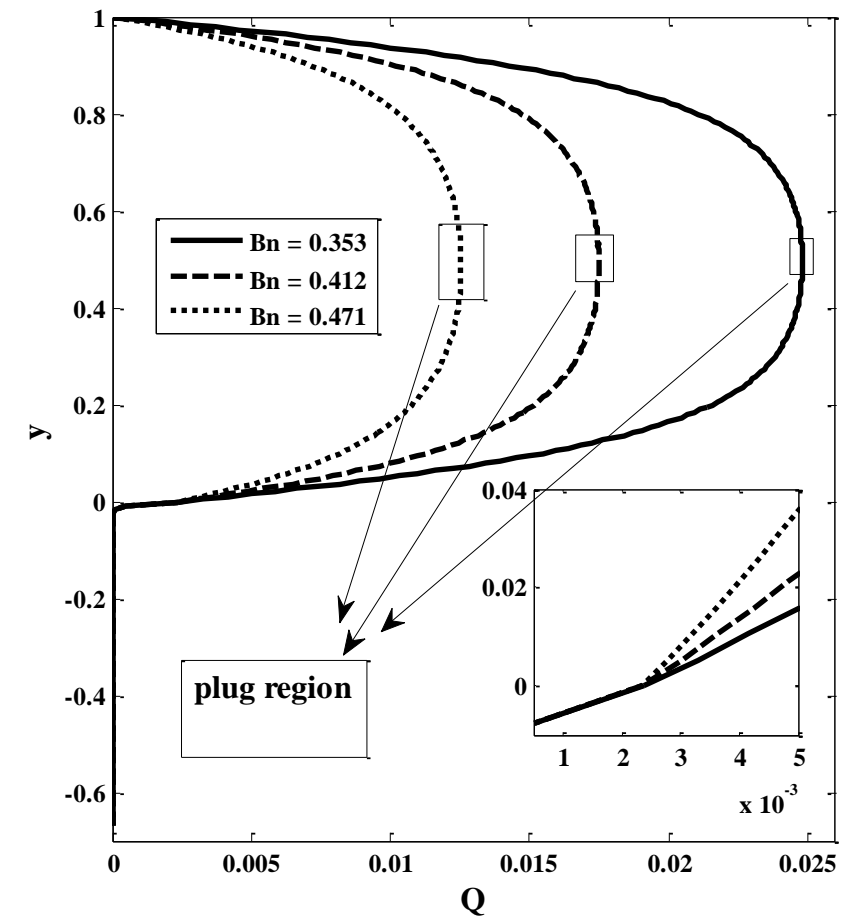

Figure B2: Effect of the Bingham number, Bn, on the basic-flow flux profiles obtained at: $\phi=0.7, \mathrm{~h}=0.67, \Pi=-1, \mathrm{~K}_{\mathrm{v}}=1, \Lambda_{\mathrm{s}}=1, \mathrm{Tx}=85, \Gamma^{-1}=0.0075$.

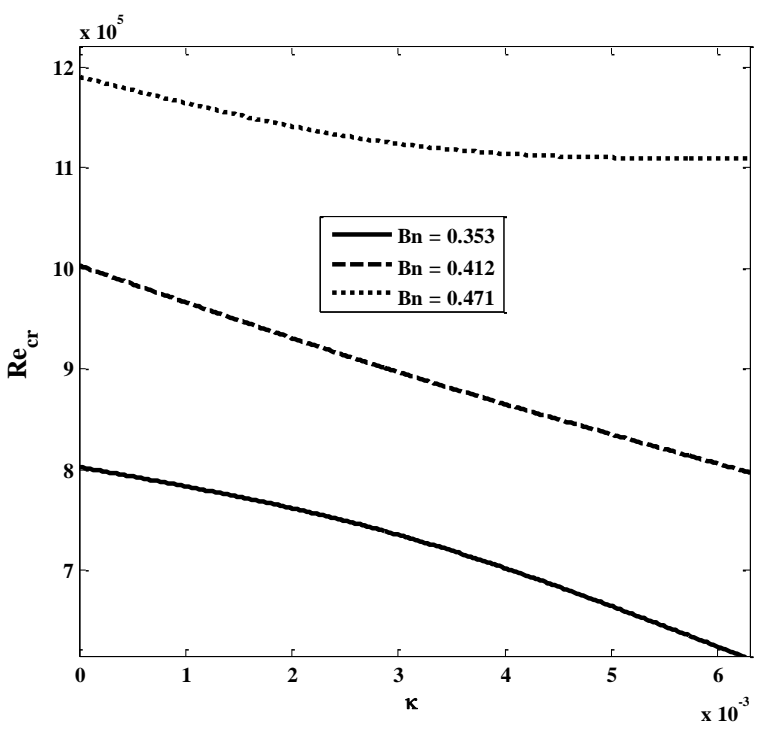

Figure B3: Effect of Bingham number on the neutral stability curve obtained at:

$$
\varphi=0.7, \mathrm{~h}=0.67, \Pi=-1, \mathrm{~K}_{\mathrm{v}}=1, \Lambda_{\mathrm{s}}=1, \mathrm{Tx}=85, \Gamma^{-1}=0.0075 \text {. }
$$




\section{Appendix C: Derivation/Solution of the Basic Solution for the M/N Case}

In this Appendix we show how the basic flow equations are derived for the N/N case and how they are numerically solved. To derive Eq. 25, we start with Eqs. 16-20. At the basic state, poroelastic region consists of fluid and solid phases both under steady conditions. Steady condition means that displacement components for the solid phase are independent of the time. Due to elastic nature of the solid, one can then conclude that:

$$
\mathrm{X}_{\mathrm{x}}=\mathrm{X}_{\mathrm{x}}(\mathrm{x}, \mathrm{y}) ; \mathrm{X}_{\mathrm{y}}=\mathrm{X}_{\mathrm{y}}(\mathrm{x}, \mathrm{y})
$$

Another key assumption for the basic state is that the flow is fully-developed both in the main channel and also in the poroelastic region - a condition necessary for the interface to remain flat at the basic state. Based on the fully-developed assumption, we have: $\mathrm{u}_{\mathrm{f}}=\mathrm{u}_{\mathrm{f}}(\mathrm{y}) ; \mathrm{v}_{\mathrm{f}}=0$ and $\mathrm{u}_{\text {core }}=\mathrm{u}_{\text {core }}(\mathrm{y}) ; \mathrm{v}_{\text {core }}=0$. Conservation of momentum in the $y$-direction between the core fluid and the fluid phase in the poroelastic region means that at the interface the pressure distribution is the same for both fluids. As such, pressure derivative in the $\mathrm{x}$-direction should also be the same. Thus we have:

$$
@ \mathrm{y}=0 \Rightarrow \sigma_{\mathrm{yy}, \mathrm{f}}=\varphi_{\mathrm{f}} \sigma_{\mathrm{yy}, \text { core }} \Rightarrow-\left.\varphi_{\mathrm{f}} \mathrm{p}_{\mathrm{avg}}\right|_{\mathrm{y}=0}+\left.\tau_{\mathrm{yy}, \mathrm{f}}\right|_{\mathrm{y}=0}=\varphi_{\mathrm{f}}\left(-\left.\mathrm{p}_{\mathrm{core}}\right|_{\mathrm{y}=0}+\left.\tau_{\mathrm{yy}}\right|_{\mathrm{y}=0}\right)
$$

and,

$$
\begin{aligned}
& \left.\tau_{\mathrm{yy}, \mathrm{f}}\right|_{\mathrm{y}=0}=2 \mathrm{~K}_{\mathrm{v}} \frac{\partial^{2} \mathrm{v}_{\mathrm{f}}}{\partial \mathrm{y}^{2}}=0 ;\left.\tau_{\mathrm{yy}, \text { core }}\right|_{\mathrm{y}=0}=2\left(1+\left.\alpha \mathrm{S}\right|_{\mathrm{y}=0}\right) \frac{\partial^{2} \mathrm{v}_{\text {core }}}{\partial \mathrm{y}^{2}}=0 \\
& \left.\Rightarrow \mathrm{p}_{\text {core }}\right|_{\mathrm{y}=0}=\left.\left.\mathrm{p}_{\mathrm{avg}}\right|_{\mathrm{y}=0} \Rightarrow \frac{\partial \mathrm{p}_{\text {core }}}{\partial \mathrm{x}}\right|_{\mathrm{y}=0}=\left.\frac{\partial \mathrm{p}_{\mathrm{avg}}}{\partial \mathrm{x}}\right|_{\mathrm{y}=0}
\end{aligned}
$$

Conservation of momentum in the x-direction between the core fluid and the fluid in the poroelastic region (i.e., Eq. 13) means that the elastic part of the solid phase in the poroelastic region is a function of "y". This can easily be deduced from the flat shape of the interface at the basic state and the notion that the lower wall of the solid phase is fixed to the lower rigid plate (see Eq. 14a). As a matter of fact, any fluctuation at the interface would mean that the horizontal and vertical components of the solid's displacement are simultaneously both $\mathrm{x}$ - and y-dependent. To achieve this goal, one can define:

$$
\mathrm{X}_{\mathrm{x}}=\mathrm{x}+\mathrm{X}_{\mathrm{x}}(\mathrm{y}) ; \mathrm{X}_{\mathrm{x}}(-\mathrm{h})=0 ; \mathrm{X}_{\mathrm{y}}=\mathrm{y}+0=\mathrm{y}
$$

The above definition means that the elastic effects can appear in the second term in the relationship for the horizontal displacement so that when this term goes to zero, the rigid solid could be recovered. Moreover, choosing the vertical coordinate as above (i.e., $\mathrm{X}_{\mathrm{y}}=\mathrm{y}$ ) satisfies the no-penetration (rigid) condition at the lower wall of the poroelastic region (i.e., $y=-h$ ). Now, by the above definitions, Eq. 16 is automatically satisfied. Equation 17 can then be written in the x-direction as:

$$
0=-\varphi_{\mathrm{f}} \frac{\partial \mathrm{p}_{\mathrm{avg}}}{\partial \mathrm{x}}+\mathrm{K}_{\mathrm{v}} \frac{\partial^{2} \mathrm{u}_{\mathrm{f}}}{\partial \mathrm{y}^{2}}+\zeta\left(0-\mathrm{u}_{\mathrm{f}}\right)
$$


where we have set $\partial \mathrm{X}_{\mathrm{x}} / \partial \mathrm{t}=0$ in the above formulation thanks to the flow/deformation being steady. In the y-direction, Eq. 17 can be written as:

$$
0=-\varphi_{\mathrm{f}} \frac{\partial \mathrm{p}_{\mathrm{avg}}}{\partial \mathrm{y}}+0 \Rightarrow \mathrm{p}_{\mathrm{avg}}=\mathrm{p}_{\mathrm{avg}}(\mathrm{x})
$$

where many terms in the left-hand-side have been set equal to zero due to the fully-developed and steady state conditions, i.e., $\partial\left(X_{x}, X_{y}\right) / \partial t=0$. Therefore, from Eqs. C3 and C6 we obtain:

$$
\left.\frac{\partial \mathrm{p}_{\text {core }}}{\partial \mathrm{x}}\right|_{\text {full core domain }}=\Pi=\left.\frac{\partial \mathrm{p}_{\mathrm{avg}}}{\partial \mathrm{x}}\right|_{\text {full poreelastic domain }} \Rightarrow \mathrm{p}_{\text {core }}=\mathrm{p}_{\mathrm{avg}}=\Pi \mathrm{x}+\mathrm{c}_{0}
$$

On the other hand, Eqs. 18 in combination with Eqs. 19 and 20 can be written in the $\mathrm{x}$ - and y-directions as:

$$
\begin{aligned}
& 0=-\varphi_{\mathrm{s}} \frac{\partial \mathrm{p}_{\mathrm{avg}}}{\partial \mathrm{x}}+\Gamma \frac{\partial^{2} \mathrm{X}_{\mathrm{x}}}{\partial \mathrm{y}^{2}}-\zeta\left(0-\mathrm{u}_{\mathrm{f}}\right) \\
& 0=-\varphi_{\mathrm{s}} \frac{\partial \mathrm{p}_{\mathrm{avg}}}{\partial \mathrm{y}}+0
\end{aligned}
$$

where, again, the solid velocity was set equal to zero due to the steady nature of the basic solution. Based on Eq. C7, it is easy to check that Eq. C9 is satisfied automatically. Now, by substituting Eq. C7 into Eqs. C5 and C8 one would obtain:

$$
\begin{aligned}
& 0=-\varphi_{\mathrm{f}} \Pi+\mathrm{K}_{\mathrm{v}} \frac{\partial^{2} \mathrm{u}_{\mathrm{f}}}{\partial \mathrm{y}^{2}}+\zeta\left(0-\mathrm{u}_{\mathrm{f}}\right) \\
& 0=-\varphi_{\mathrm{s}} \Pi+\Gamma \frac{\partial^{2} \mathrm{X}_{\mathrm{x}}}{\partial \mathrm{y}^{2}}-\zeta\left(0-\mathrm{u}_{\mathrm{f}}\right)
\end{aligned}
$$

Now, to solve the above equations we start with solving Eq. 25 (or Eq. C8) first. By so-doing, the following equation is obtained for the horizontal velocity of the fluid phase in the poroelastic layer:

$$
\mathrm{u}_{\mathrm{f}}(\mathrm{y})=-\frac{\varphi_{\mathrm{f}}}{\zeta} \Pi+\mathrm{c}_{1} \cosh \left(\sqrt{\frac{\zeta}{\mathrm{K}_{\mathrm{v}}} \mathrm{y}}\right)+\mathrm{c}_{2} \sinh \left(\sqrt{\frac{\zeta}{\mathrm{K}_{\mathrm{v}}} \mathrm{y}}\right)
$$

where $c_{1}$ and $c_{2}$ are constants of integration. Substituting this equation into Eq. 26, we obtain the following expression for the horizontal displacement of the solid phase in the poroelastic layer:

$$
\mathrm{X}_{\mathrm{x}}(\mathrm{y})=\frac{\Pi}{2 \Gamma} \mathrm{y}^{2}+\mathrm{c}_{3} \mathrm{y}+\mathrm{c}_{4}-\frac{\mathrm{K}_{\mathrm{v}}}{\Gamma} \mathrm{c}_{1} \cosh \left(\sqrt{\frac{\zeta}{\mathrm{K}_{\mathrm{v}}} \mathrm{y}}\right)-\frac{\mathrm{K}_{\mathrm{v}}}{\Gamma} \mathrm{c}_{2} \sinh \left(\sqrt{\frac{\zeta}{\mathrm{K}_{\mathrm{v}}} \mathrm{y}}\right)
$$


where $\mathrm{c}_{3}$ and $\mathrm{c}_{4}$ are new constants of integration. The above four constants of integration can be obtained using the boundary condition as given by Eq. 29; that is:

$$
\begin{aligned}
& \mathrm{u}_{\mathrm{f}}(-\mathrm{h})=0 \stackrel{(\mathrm{Cl} 2)}{\Rightarrow}-\frac{\varphi_{\mathrm{f}}}{\zeta} \Pi+\mathrm{c}_{1} \cosh \left(\sqrt{\frac{\zeta}{\mathrm{K}_{\mathrm{v}}}} \mathrm{h}\right)-\mathrm{c}_{2} \sinh \left(\sqrt{\frac{\zeta}{\mathrm{K}_{\mathrm{v}}}} \mathrm{h}\right)=0 \\
& \mathrm{X}_{\mathrm{x}}(-\mathrm{h})=0 \stackrel{(\mathrm{Eg} \cdot \mathrm{Cl} 13)}{\Rightarrow} \frac{\Pi}{2 \Gamma} \mathrm{h}^{2}-\mathrm{c}_{3} \mathrm{~h}+\mathrm{c}_{4}-\frac{\mathrm{K}_{\mathrm{v}}}{\Gamma} \mathrm{c}_{1} \cosh \left(\sqrt{\frac{\zeta}{\mathrm{K}_{\mathrm{v}}}} \mathrm{h}\right)+\frac{\mathrm{K}_{\mathrm{v}}}{\Gamma} \mathrm{c}_{2} \sinh \left(\sqrt{\frac{\zeta}{\mathrm{K}_{\mathrm{v}}}} \mathrm{h}\right)=0
\end{aligned}
$$

At the basic state, the dimensionless stress tensor for the fluid in the main channel can be written as:

$$
\tau=(1+\varepsilon S)^{\beta} 2 D \Rightarrow\left[\begin{array}{cc}
\tau_{x x} & \tau_{x y} \\
\tau_{y x} & \tau_{y y}
\end{array}\right]=(1+\varepsilon S)^{\beta}\left[\begin{array}{cc}
0 & \frac{d u}{d y} \\
\frac{d u}{d y} & 0
\end{array}\right]
$$

Using the stress boundary conditions in Eq. 29, we have:

$$
\begin{aligned}
& \tau_{x y, f}(0)=\left.\varphi_{f} \tau_{x y}(0) \Rightarrow K_{v} \frac{d u_{f}}{d y}\right|_{y=0}=\left.\varphi_{f}\left[1+\left.\varepsilon S\right|_{y=0}\right]^{\beta} \frac{d u}{d y}\right|_{y=0} \\
& \Rightarrow \sqrt{\zeta K_{v}} c_{2}=\left.\varphi_{f}\left[1+\left.\varepsilon S\right|_{y=0}\right]^{\beta} \frac{d u}{d y}\right|_{y=0} \\
& \tau_{x y, s}(0)=\left.\varphi_{s} \tau_{x y}(0) \Rightarrow \Gamma \frac{d X_{x}}{d y}\right|_{y=0}=\left.\varphi_{s}\left[1+\left.\varepsilon S\right|_{y=0}\right]^{\beta} \frac{d u}{d y}\right|_{y=0} \\
& \Rightarrow c_{3}-\frac{\sqrt{\zeta K_{v}}}{\Gamma} c_{2}=\left.\varphi_{s}\left[1+\left.\varepsilon S\right|_{y=0}\right]^{\beta} \frac{d u}{d y}\right|_{y=0}
\end{aligned}
$$

Equations C14, C15, C17, and C18 must be solved simultaneously to obtain the unknown constants, $\mathrm{c}_{1}, \mathrm{c}_{2}, \mathrm{c}_{3}, \mathrm{c}_{4}$. The problem is that the velocity gradient (which is coupled with the structural parameter at the interface) appears in these equations and it is an unknown entity at this stage. To circumvent this problem, we resort to the flow through the main channel. From Equations 21 and $22 \mathrm{~b}$ we obtain:

$$
\begin{aligned}
& \stackrel{(\text { Eq. 21) }}{\Rightarrow} 0=-\frac{\partial p}{\partial x}+\frac{\partial \tau_{x y}}{\partial y} \Rightarrow 0=-\Pi+\frac{\partial \tau_{x y}}{\partial y} \Rightarrow \tau_{x y}=\Pi y+c_{5} \\
& \stackrel{(\text { Eq. 22b) }}{\Rightarrow} 0=-T x\left|\frac{d u}{d y}\right| S+1-S \Rightarrow S=\frac{1}{1+T x\left|\frac{d u}{d y}\right|}
\end{aligned}
$$

where $c_{5}$ is a new constant of integration. Substituting the shear stress from Eq. C16 into Eq. C19 we obtain: 


$$
(1+\varepsilon S)^{\beta} \frac{\mathrm{du}}{\mathrm{dy}}=\Pi y+\mathrm{c}_{5}
$$

Substituting Eq. C20 into Eq. C21, we obtain the following nonlinear ordinary differential equation:

$$
\frac{\mathrm{du}}{\mathrm{dy}}=\left(\Pi y+\mathrm{c}_{5}\right)\left(1+\frac{\varepsilon}{1+\mathrm{Tx}\left|\frac{\mathrm{du}}{\mathrm{dy}}\right|}\right)^{-\beta} \text {. }
$$

This equation can be solved subject to the no-slip condition at $y=1$; that is: $u(1)=0$. Based on the mass conservation law at the interface, an extra boundary condition can be obtained (see the third condition in Eq. 29) as:

$$
\Rightarrow \varphi_{\mathrm{f}} \Lambda_{\mathrm{f}} \mathrm{u}_{\mathrm{f}}(0)=\mathrm{u}(0) \Rightarrow \varphi_{\mathrm{f}} \Lambda_{\mathrm{f}}\left[-\frac{\varphi_{\mathrm{f}}}{\zeta} \Pi+\mathrm{c}_{1}\right]=\mathrm{u}(0)
$$

It is worth-mentioning that, thanks to the steady assumption of the basic state, the solid part is absent from the mass-conservation-law at the interface. Equation C22 subjected to the above boundary conditions has no analytical solution in close sight, and so we resort to numerical methods for this purpose. Due to the presence of $c_{1}$ in Eq. C23, even a numerical solution is not straightforward simply because these equations must be solved simultaneously. Before doing anything, we try to satisfy Eq. C1 at the interface $(\mathrm{y}=0)$ and then substitute it into Eqs. C17 and C18. The result is:

$$
\begin{gathered}
\left.\Rightarrow\left[(1+\varepsilon \lambda)^{\beta} \frac{\mathrm{du}}{\mathrm{dy}}\right]\right|_{\mathrm{y}=0}=\left.\left(\Pi y+\mathrm{c}_{5}\right)\right|_{\mathrm{y}=0} \stackrel{(\text { Eq. C17) }}{\Rightarrow} \sqrt{\zeta \mathrm{K}_{\mathrm{v}}} \mathrm{c}_{2}=\varphi_{\mathrm{f}} \mathrm{c}_{5} \Rightarrow \\
{\left.\left[(1+\varepsilon \lambda)^{\beta} \frac{\mathrm{du}}{\mathrm{dy}}\right]\right|_{\mathrm{y}=0}=\left.\left(\Pi y+\mathrm{c}_{5}\right)\right|_{\mathrm{y}=0} \stackrel{(\text { Eq. C18) }}{\Rightarrow} \mathrm{c}_{3}-\frac{\sqrt{\zeta \mathrm{K}_{\mathrm{v}}}}{\Gamma} \mathrm{c}_{2}=\varphi_{\mathrm{s}} \mathrm{c}_{5}}
\end{gathered}
$$

We finally obtain:

$$
\left[\begin{array}{cccc}
\cosh \left(\sqrt{\frac{\zeta}{\mathrm{K}_{\mathrm{v}}} \mathrm{h}}\right) & -\sinh \left(\sqrt{\frac{\zeta}{\mathrm{K}_{\mathrm{v}}} \mathrm{h}}\right) & 0 & 0 \\
-\frac{\mathrm{K}_{\mathrm{v}}}{\Gamma} \cosh \left(\sqrt{\frac{\zeta}{\mathrm{K}_{\mathrm{v}}} \mathrm{h}}\right) & \frac{\mathrm{K}_{\mathrm{v}}}{\Gamma} \sinh \left(\sqrt{\frac{\zeta}{\mathrm{K}_{\mathrm{v}}} \mathrm{h}}\right) & -\mathrm{h} & 1 \\
0 & \sqrt{\zeta \mathrm{K}_{\mathrm{v}}} & 0 & 0
\end{array}\right]\left[\begin{array}{l}
\mathrm{c}_{1} \\
\mathrm{c}_{2} \\
\mathrm{c}_{3} \\
\mathrm{c}_{4}
\end{array}\right]=\left[\begin{array}{c}
\frac{\varphi_{\mathrm{f}}}{\zeta} \Pi \\
\frac{\Pi}{2 \Gamma} \mathrm{h}^{2} \\
\varphi_{\mathrm{f}} \mathrm{c}_{5} \\
\varphi_{\mathrm{s}} \mathrm{c}_{5}
\end{array}\right]
$$

To proceed with our solution scheme, an arbitrary guess is made for the constant $\mathrm{c}_{5}$. Then the matrix equation is solved to obtain the constants $\mathrm{c}_{1}, \mathrm{c}_{2}, \mathrm{c}_{3}, \mathrm{c}_{4}$. As the next stage, Eq. C22 is numerically solved 
subject to no-slip boundary condition at $\mathrm{y}=1$ such that $\mathrm{u}(1)=0$. As soon as the velocity field is obtained in the main channel, it is checked to see if the other boundary condition, i.e., Eq. C20, is satisfied. If not, the guess is revised. This technique should render the remaining constants after a couple of iterations. Eventually, a complete solution is obtained for the velocity profile and the displacement in the poroelastic region. Similarly, the horizontal velocity profile can be obtained for the main channel.

\section{Appendix D: Results for the Quemada Model}

The superiority of the Quemada model over the Moore model is that, unlike Moore model, it allows a nonlinear relationship between the viscosity and the structural parameter typical of the real world. For example, in his seminal papers [37,38] Quemada have shown that the following viscosity function fits the data for physiological fluids such as blood quite well [39]; that is:

$$
\eta(\lambda, \dot{\gamma})=\mu_{\infty}(1+\varepsilon S)^{-2}
$$

Another important feature of the Quemada model is that, unlike Moore model which represents thixotropic fluids only, it can also represent anti-thixotropic (or, rheopectic) fluids such as synovia. To achieve this goal, it suffices that in Eq. D1, a proper sign is adopted for the viscosity-gap ratio, $\varepsilon$. This notion can best be seen in Fig. D1 which shows the time-evolution of the apparent viscosity for $\varepsilon=+0.5$ and $\varepsilon=-0.5$. This figure clearly shows that for $\varepsilon>0$ the response becomes typical of anti-thixotropic fluids whereas for $\varepsilon<0$ the response is that of shear-thinning fluids. It needs to be mentioned that to obtain these results, and other results to be presented shortly, the parameters in the Quemada model were set at: $\beta=-2, \mathrm{~m}=0, \mathrm{n}=0.5$ as suggested by Frigaard [51].

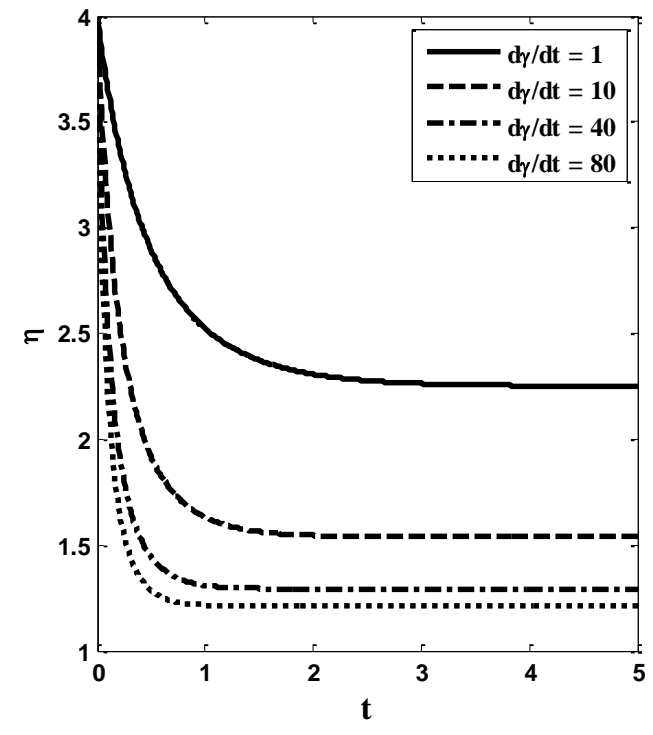

(a)

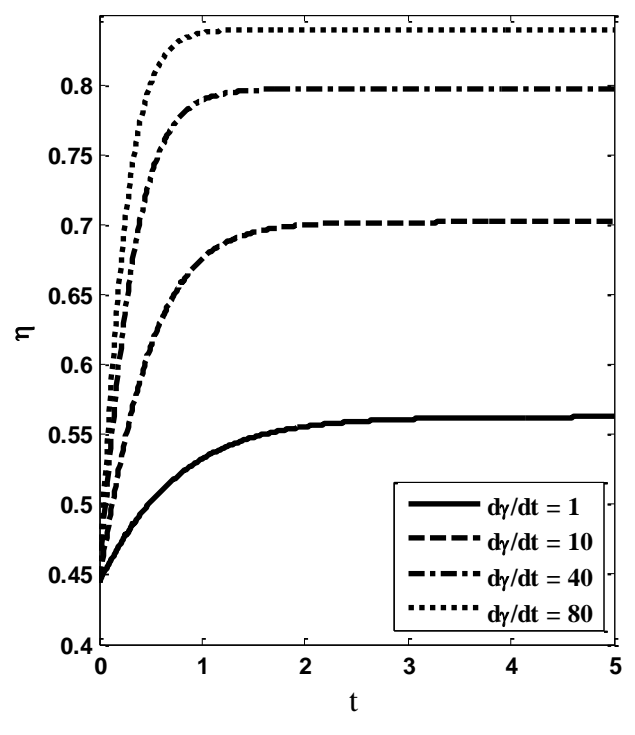

(b)

Figure D1: Time variation of the viscosity function for the $\mathrm{Q} / \mathrm{N}$ case obtained at $\mathrm{Tx}=0.5$ : 
(a) thixotropic behavior at $\varepsilon=-0.5$, (b) anti-thixotropic behavior at $\varepsilon=+0.5$.

Figure D2 shows typical basic-flow flux profiles obtained for the Quemada model based on Eq. D1 with the model parameters set at: $\mathrm{m}=0, \mathrm{n}=0.5$ As mentioned above, a negative $\varepsilon$ corresponds to shearthinning fluids whereas a positive $\varepsilon$ means shear-thickening. This figure shows that the flux is larger for the shear-thinning case, as expected. This figure also shows that by an increase in the Tx number, the flux is decreased for the shear-thickening case, again, as expected.

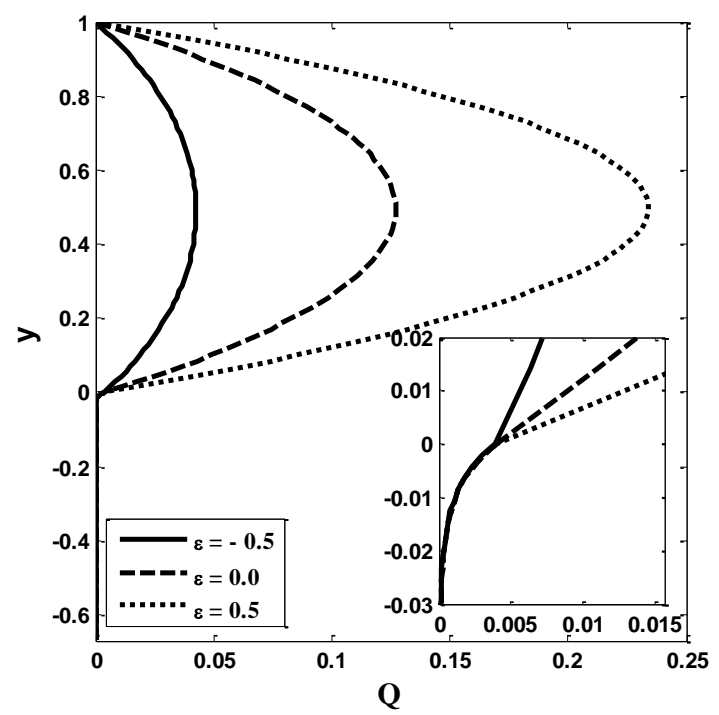

(a)

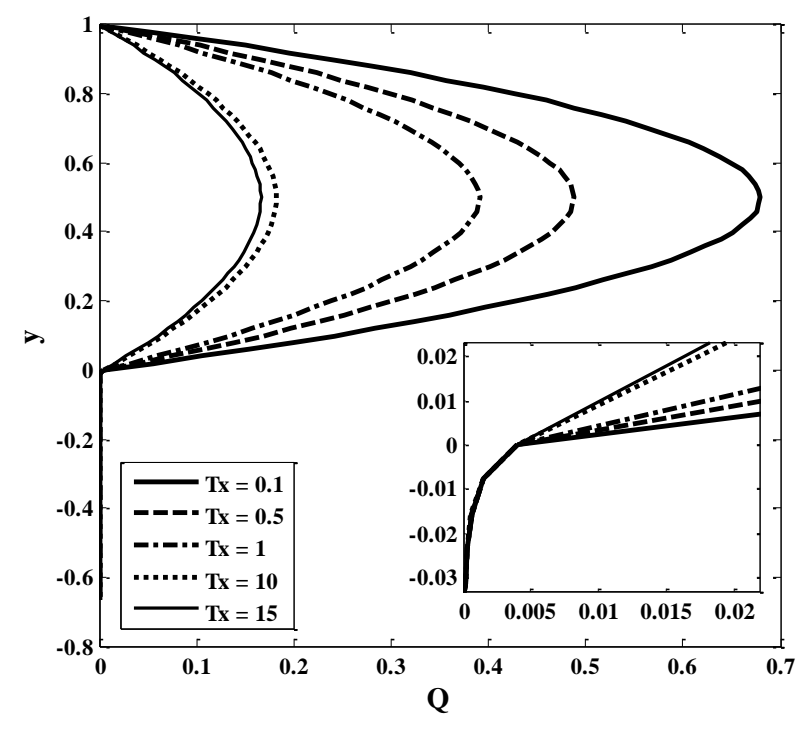

(b)

Figure D2: Basic flow flux profile for the $\mathrm{Q} / \mathrm{N}$ case obtained at $\varphi=0.7, \kappa=0.01, \Gamma^{-1}=0.009, \Pi=-1, \mathrm{~h}=0.67, \Lambda_{\mathrm{f}}=\Lambda_{\mathrm{s}}=1$ :

(a) effect of the viscosity-gap ratio, $\varepsilon$, obtained for $\mathrm{Tx}=0.5$,

(b) effect of thixotropy number, Tx, obtained for $\varepsilon=1.5$.

Having found the basic solution, we can now investigate their stability to small perturbations. For Quemada model, the coefficients in Eq. 38a turn out to be [see Ref. 33]:

$$
\begin{aligned}
& \mathrm{b}_{1}(\mathrm{y})=\frac{1}{\mathrm{i \alpha}} \operatorname{sgn}\left(\left|\frac{\mathrm{d} \overline{\mathrm{u}}}{\mathrm{dy}}\right|\right)\left\{\mathrm{m}(1-\overline{\mathrm{S}}(\mathrm{y}))\left|\frac{\mathrm{d} \overline{\mathrm{u}}}{\mathrm{dy}}\right|^{\mathrm{m}-1}-\mathrm{n} \mathrm{Tx} \overline{\mathrm{S}}(\mathrm{y})\left|\frac{\mathrm{d} \overline{\mathrm{u}}}{\mathrm{dy}}\right|^{\mathrm{n}-1}\right\} \\
& \mathrm{b}_{2}(\mathrm{y})=\frac{1}{\Theta} \frac{\mathrm{d} \overline{\mathrm{S}}}{\mathrm{dy}}+\operatorname{sgn}\left(\left|\frac{\mathrm{d} \overline{\mathrm{u}}}{\mathrm{dy}}\right|\right) \mathrm{i \alpha}\left\{\mathrm{nTx} \overline{\mathrm{S}}(\mathrm{y})\left|\frac{\mathrm{d} \overline{\mathrm{u}}}{\mathrm{dy}}\right|^{\mathrm{n}-1}+\mathrm{m}(\overline{\mathrm{S}}(\mathrm{y})-1)\left|\frac{\mathrm{d} \overline{\mathrm{u}}}{\mathrm{dy}}\right|^{\mathrm{m}-1}\right\} \\
& \mathrm{b}_{3}(\mathrm{y})=\frac{\omega}{\Theta}+\mathrm{Tx}\left|\frac{\mathrm{d} \overline{\mathrm{u}}}{\mathrm{dy}}\right|^{\mathrm{n}}+\left|\frac{\mathrm{d} \overline{\mathrm{u}}}{\mathrm{dy}}\right|^{\mathrm{m}}+\frac{\mathrm{i} \alpha}{\Theta} \overline{\mathrm{u}}(\mathrm{y})
\end{aligned}
$$


where, as mentioned above, we set: $\mathrm{m}=0, \mathrm{n}=0.5$. Similarly, as to the perturbed stress tensor for the Quemada fluid in the main channel, Eq. 53c becomes [33]:

$\hat{\tau}=\left[\begin{array}{c}2 i \alpha[1+\varepsilon \bar{S}(y)]^{\beta} \hat{u}(y) \\ \left\{\begin{array}{c}{[1+\varepsilon \bar{S}(y)]^{\beta}\left(\frac{d \hat{u}}{d y}+i \alpha \hat{v}(y)\right)} \\ +\varepsilon \beta \frac{d \bar{u}}{d y} \hat{\lambda}(y) \\ +\varepsilon \beta \frac{d \bar{u}}{d y} \hat{\lambda}(y)\end{array}\right\} \\ 2[1+\varepsilon \bar{S}(y)]^{\beta} \frac{d \hat{v}}{d y}\end{array}\right] e^{i \alpha x+\omega t}$

where $\beta=-2$. In Figs. D3 and D4 we have addressed the effect of the flexibility and permeability numbers, respectively, on the critical Reynolds number. As can be seen in Fig. D3, by an increase in the flexibility of the porous layer, the flow becomes progressively more stable whether it is thixotropic or anti-thixotropic. Still, at any given flexibility parameter, the anti-thixotropic fluid is more stable. The effect of permeability factor is more intriguing, as can be seen in Fig. D4. That is, for thixotropic fluids (i.e., for $\varepsilon<0$ ) the effect of permeability can be stabilizing or destabilizing depending on its magnitude. For anti-thixotropic fluids $(\varepsilon>0)$, however, the effect of permeability is predicted to be monotonically destabilizing.

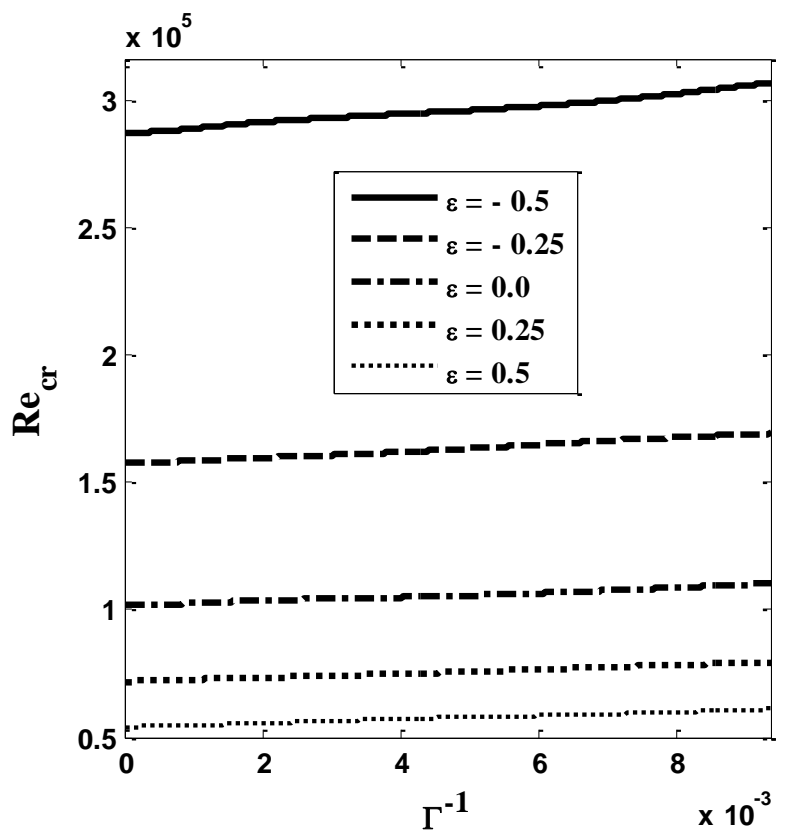

Figure D3: Critical Reynolds number as a function of the flexibility parameter for the $\mathrm{Q} / \mathrm{N}$ case obtained at: $\varphi=0.7, \mathrm{Tx}=0.5, \Pi=-1, \mathrm{~h}=0.67, \kappa=0.01, \Lambda_{\mathrm{f}}=1, \Lambda_{\mathrm{s}}=1, \mathrm{~K}_{\mathrm{v}}=1$. 


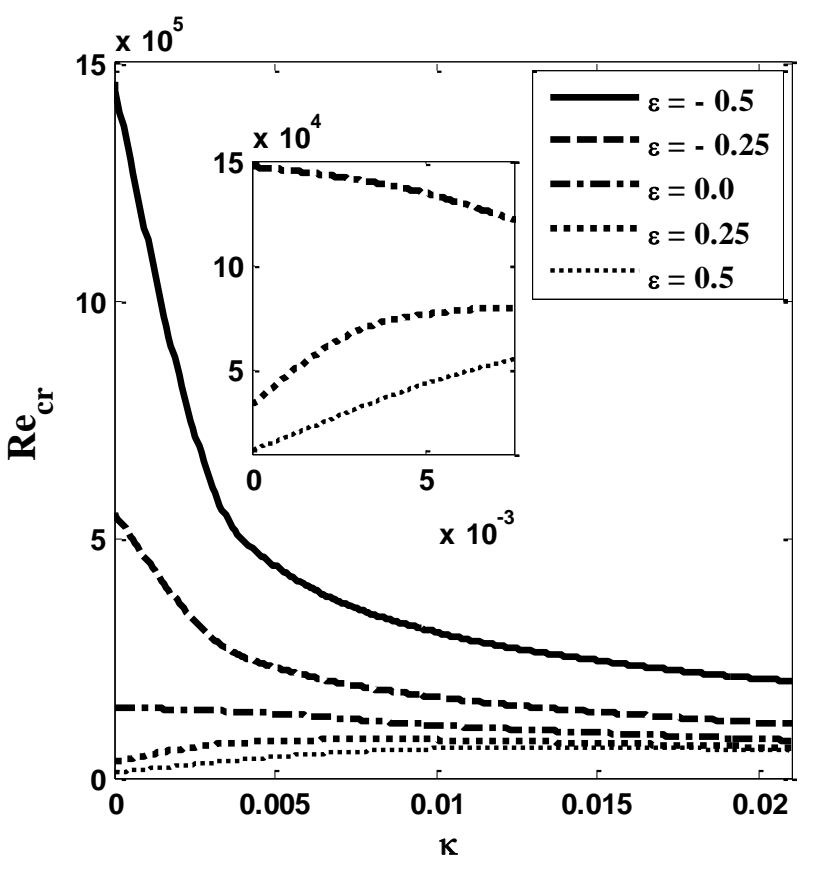

Figure D4: Critical Reynolds number as a function of the permeability number for the $\mathrm{Q} / \mathrm{N}$ case obtained at: $\varphi=0.7, \mathrm{Tx}=0.5, \Pi=-1, \mathrm{~h}=0.67, \Gamma^{-1}=0.009, \Lambda_{\mathrm{f}}=1, \Lambda_{\mathrm{s}}=1, \mathrm{~K}_{\mathrm{v}}=1$. 